\title{
Heteronuclear spin decoupling in solid-state NMR under magic- angle sample spinning
}

\section{Habilitation Thesis}

Author(s):

Ernst, Matthias (D)

Publication date:

2002

Permanent link:

https://doi.org/10.3929/ethz-a-004444467

Rights / license:

In Copyright - Non-Commercial Use Permitted 


\title{
Heteronuclear Spin Decoupling in Solid-State NMR \\ Under Magic-Angle Sample Spinning
}

\author{
HABILITATIONSSCHRIFT
}

vorgelegt dem Departement Chemie

der Eidgenössischen Technischen Hochschule Zürich

von

Dr. Matthias Ernst 



\section{Table of Contents}

Table of Contents $\quad$ i

$\begin{array}{ll}\text { Abstract } & 1\end{array}$

1 Introduction 3

1.1 General Motivation 3

$1.2 \quad$ High-Power CW Decoupling 8

$1.3 \quad$ High-Power Multiple-Pulse Decoupling 11

$1.4 \quad$ Low-Power Decoupling 13

1.5 General Theoretical Framework 14

1.6 References 16

$2 \quad$ High-Power CW Decoupling in Isolated Spin Systems 21

2.1 Theory 21

2.1.1 Sequential Averaging Approach $\quad 22$

2.1.2 Simultaneous Averaging of Space and Spin Parts 29

2.2 Numerical Simulations 30

$2.3 \quad$ Experimental Results 34

2.4 References 39

$3 \quad$ High-Power CW Decoupling in Strongly-Coupled Spin Systems 41

3.1 Theory 41

3.2 Numerical Simulations 46

3.3 Comparison Between Experiments and Simulations 47

3.4 References 51

$4 \quad$ Low-Power CW Decoupling 53

4.1 Theory 53

4.2 Numerical Simulations 58

4.3 Experimental Results and Discussion 60

$\begin{array}{lll}4.4 & \text { References } & 67\end{array}$

$5 \quad$ Multiple-Pulse Decoupling Under MAS 69

$\begin{array}{lll}5.1 & \text { General Considerations } & 69\end{array}$ 
$\begin{array}{lll}5.2 & \text { TPPM Decoupling } & 70\end{array}$

$\begin{array}{lll}5.3 & \text { XiX Decoupling } & 74\end{array}$

$\begin{array}{lll}\text { 5.3.1 Theory and Numerical Simulations } & 74\end{array}$

5.3.2 Experimental Data and Discussion 76

5.4 Comparison between TPPM and XiX Decoupling 79

5.5 References 83

$\begin{array}{lll}6 & \text { Conclusions and Outlook } & 85\end{array}$

6.1 References 88

$\begin{array}{llr}7 & \text { Appendices } & 89\end{array}$

$\begin{array}{lll}7.1 & \text { Appendix A } & 89\end{array}$

$\begin{array}{lll}7.2 & \text { Appendix B } & 92\end{array}$

$7.3 \quad$ References 94

$\begin{array}{ll}\text { Acknowledgements } & 95\end{array}$ 


\begin{abstract}
Achieving high spectral resolution is an important prerequisite for the application of solid-state NMR to biological molecules. Higher spectral resolution allows to resolve a larger number of resonances and leads to higher sensitivity. Among other things, heteronuclear spin decoupling is one of the important factors which determine the resolution of a spectrum. The process of heteronuclear spin decoupling under magic-angle sample spinning is analyzed in detail. Continuouswave rf irradiation leads only in a zeroth-order approximation to a full decoupling of heteronuclear spin systems in solids under magic-angle spinning (MAS). In a higherorder approximation, a cross-term between the dipolar-coupling tensor and the chemical-shielding tensor is reintroduced, providing a scaled coupling term between the heteronuclear spins. In strongly coupled spin systems this second-order recoupling term is partially averaged out by the proton spin-diffusion process, which leads to exchange-type narrowing of the line by proton spin flips. This process can be described by a spin-diffusion type superoperator, allowing the efficient simulation of strongly-coupled spin systems under heteronuclear spin decoupling. Low-power continuous-wave decoupling at fast MAS frequencies offers an alternative to highpower irradiation by reversing the order of the averaging processes. At fast MAS frequencies low-power continuous-wave decoupling leads to significantly narrower lines than high-power continuous-wave decoupling while at the same time reducing the power dissipated in the sample by several orders of magnitude. The best decoupling is achieved by multiple-pulse sequences at high rf fields and under fast MAS. Two such sequences, two-pulse phase-modulated decoupling (TPPM) and Xinverse- $X$ decoupling $(\mathrm{X} \mathrm{i})$, are discussed and their properties analyzed and compared.
\end{abstract}




\section{Introduction}

\subsection{General Motivation}

High spectral resolution is an essential prerequisite for the application of solidstate nuclear magnetic resonance (NMR) spectroscopy to larger biological systems. Unambiguous assignment of resonances is only possible if the resonances can be distinguished in a multi-dimensional correlation spectrum. Based on the assignment, localized information about distances or torsion angles can then be obtained which form the basis for any structural constraints obtained from solid-state NMR. Reducing the linewidth allows not only a larger number of resonance to be resolved but, at the same time, leads to a higher signal-to-noise ratio since the integral of the line remains constant.

In NMR spectroscopy, one of the most important tools for obtaining narrow lines and for simplifying the spectrum is heteronuclear spin decoupling (1). Originally, continuous-wave (cw) decoupling was used in liquid-state NMR, but it was soon realized that resonance offsets could lead to incomplete decoupling and residual line splittings (2),(3). To reduce this problem, noise decoupling (4) was introduced where a pseudo-random phase inversion was applied to the radiofrequency (rf) irradiation. Today, one usually decouples the isotropic heteronuclear Jcoupling in liquid-state NMR by applying a multiple-pulse sequence such as MLEV (5), WALTZ (6),(7), DIPSI (8), or GARP (9) or by using adiabatic inversion pulses like WURST (10) or SWIRL (11). Since the J-coupling constants are often quite small, e.g., less than $200 \mathrm{~Hz}$ in organic solids, one can use relatively low rf-field strengths to obtain broadband heteronuclear decoupling.

In solid-state NMR, the situation concerning heteronuclear spin decoupling is more complicated. We do not only have to consider the isotropic heteronuclear Jcoupling but also the anisotropic heteronuclear dipolar coupling, which is in many cases several orders of magnitude larger than the J-coupling. Under magic-angle sample spinning (MAS) the anisotropic heteronuclear dipolar coupling becomes time 
dependent and leads to a sideband pattern as does the anisotropic chemical-shielding tensor. If one now imposes an additional time dependence on the spin system due to rf irradiation, one can get interference effects between the two time-dependent processes if they are on the same time scale. These interference effects can manifest themselves as undesired recoupling of the dipolar interaction $(12),(13),(14),(15),(16)$ which can lead to a significant broadening of the lines.

The situation becomes even more complicated in the presence of strong homonuclear dipolar couplings. The homonuclear dipolar-coupling Hamiltonian does not commute with itself at different points in time under MAS and also not with the heteronuclear dipolar-coupling Hamiltonian. This property leads to an incomplete averaging of the heteronuclear dipolar couplings by MAS. The homonuclear dipolar-coupling Hamiltonian can also lead to a spin-diffusion type magnetization-transfer process among the irradiated spins, which can also interfere with the decoupling process. The term spin diffusion in the present context characterizes a generalized polarization-transfer process between the I spins which is propagated by flip-flop processes. The Hamiltonian for these flip-flop processes can either be the normal static dipolar-coupling Hamiltonian or the first-order dipolarcoupling average Hamiltonian under MAS. These differences between the liquid-state and the solid-state NMR Hamiltonians explain why efficient heteronuclear spin decoupling in solids is more demanding and difficult to achieve than in liquid-state NMR.

For several decades until about 1995, high-power cw decoupling remained the most common way to achieve heteronuclear spin decoupling in solid-state NMR under MAS (17),(18),(19). The introduction of two-pulse phase-modulated decoupling (TPPM) by Bennett et al. (20) initiated renewed interest in the understanding of the decoupling process in rotating solids and started the development of more advanced decoupling sequences.

If one could spin a solid powder sample containing only spin-1/2 nuclei infinitely fast about the magic angle, one would basically expect to recover the highresolution spectrum obtained in liquid-state NMR. All anisotropic second-rank tensor 
interactions would be averaged out, and the decoupling problem would then be reduced to decoupling the isotropic J interaction. To implement such an approach would, however, require considerably higher MAS frequencies than are available today (2001). Figure 1.1 shows ${ }^{13} \mathrm{C}$ spectra of a $\mathrm{CH}$ group (Figure 1.1a, selectively labeled $2-{ }^{13} \mathrm{C}$-alanine) and a $\mathrm{CH}_{2}$ group (Figure $1.1 \mathrm{~b}$, selectively labeled $2-{ }^{13} \mathrm{C}$ glycine) at MAS spinning frequencies between 10 and $50 \mathrm{kHz}$ obtained without proton irradiation. At $50 \mathrm{kHz}$ spinning frequency the linewidth of the $\mathrm{CH}$ group was found to be about $250 \mathrm{~Hz}$ full width at half height (FWHH) while the minimum linewidth reached in this sample using high-power TPPM decoupling was about $35 \mathrm{~Hz}$ FWHH. In the $\mathrm{CH}_{2}$ group the linewidth at $50 \mathrm{kHz}$ MAS spinning frequency was about $750 \mathrm{~Hz}$ while the minimum linewidth reached using TPPM decoupling was $50 \mathrm{~Hz}$. Figure 1.2 shows the measured line width (FWHH) plotted as a function of the spinning frequency for the $\mathrm{CH}$ group (circles) and for the $\mathrm{CH}_{2}$ group (squares). The solid lines in Figure 1.2 show the best two fits of the function $f\left(v_{r}\right)=a / v_{r}+b$ to

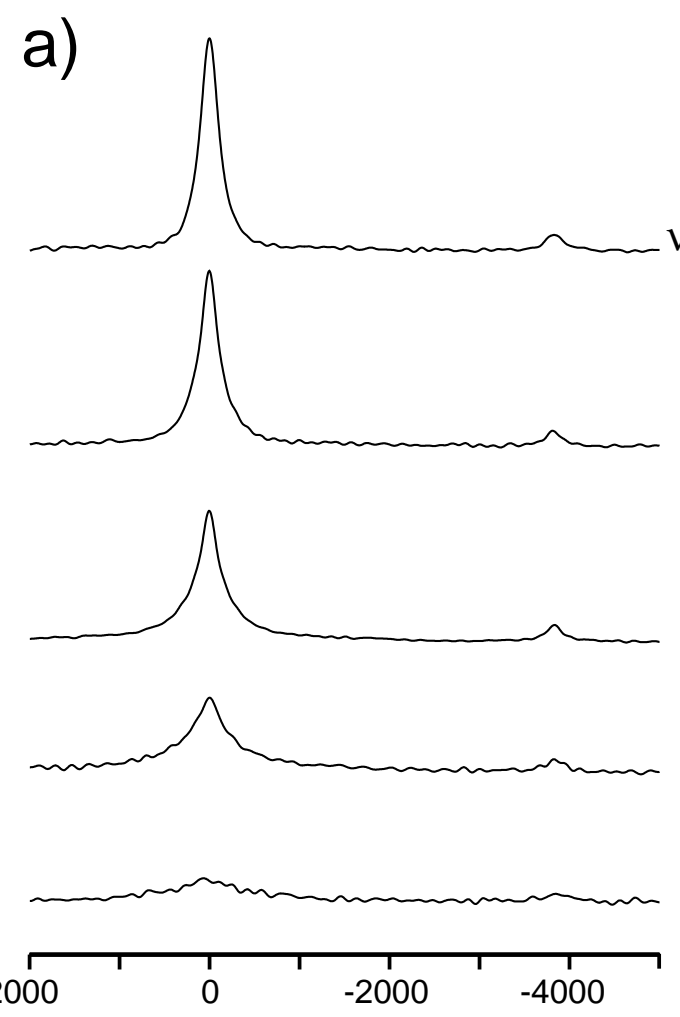

$[\mathrm{Hz}]$

b) $v_{\mathrm{r}}=50 \mathrm{kHz}$

$40 \mathrm{kHz}$

$30 \mathrm{kHz}$ $20 \mathrm{kHz}$ $10 \mathrm{kHz}$

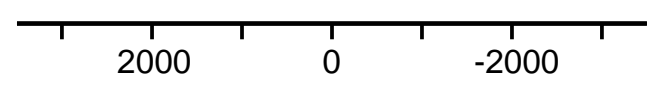

$[\mathrm{Hz}]$

Figure 1.1: ${ }^{13} \mathrm{C}$ spectra of $2-{ }^{13} \mathrm{C}$-alanine (a) and $2-{ }^{13} \mathrm{C}$-glycine (b) at different MAS spinning frequencies without proton irradiation. 
the measured data. The experimental linewidths follow quite well a $1 / \mathrm{v}_{\mathrm{r}}$ dependence as one would expect from theoretical considerations (see Chapter 4.1).

From the spectra shown in Figure 1.1 it can clearly be seen that with current MAS technology spinning without rf irradiation is not sufficient to obtain dipolardecoupled carbon spectra in a powder sample of a typical organic solid. In order to average out the residual dipolar couplings and the heteronuclear J-couplings, which are invariable under MAS, rf irradiation is needed. Adding rf irradiation to MAS generates complications due to possible interference effects between the physical rotation of the sample and the rotation of the spins by the applied cw rf-field. The most important interference effects in the context of decoupling are rotary-resonance recoupling at $\mathrm{n} \cdot \omega_{\mathrm{r}}=\omega_{1}$ with $\mathrm{n}=1,2(12),(13),(14),(15)$ and the HORROR condition at $\frac{1}{2} \cdot \omega_{\mathrm{r}}=\omega_{1}$ (16). The first leads to a recoupling of the heteronuclear dipolar coupling while the latter leads to a recoupling of the homonuclear dipolar interaction. In principle, there are also higher rotary-resonance conditions with $n>2$ but they are usually weak. To avoid interference from these rotary-resonance conditions, it is

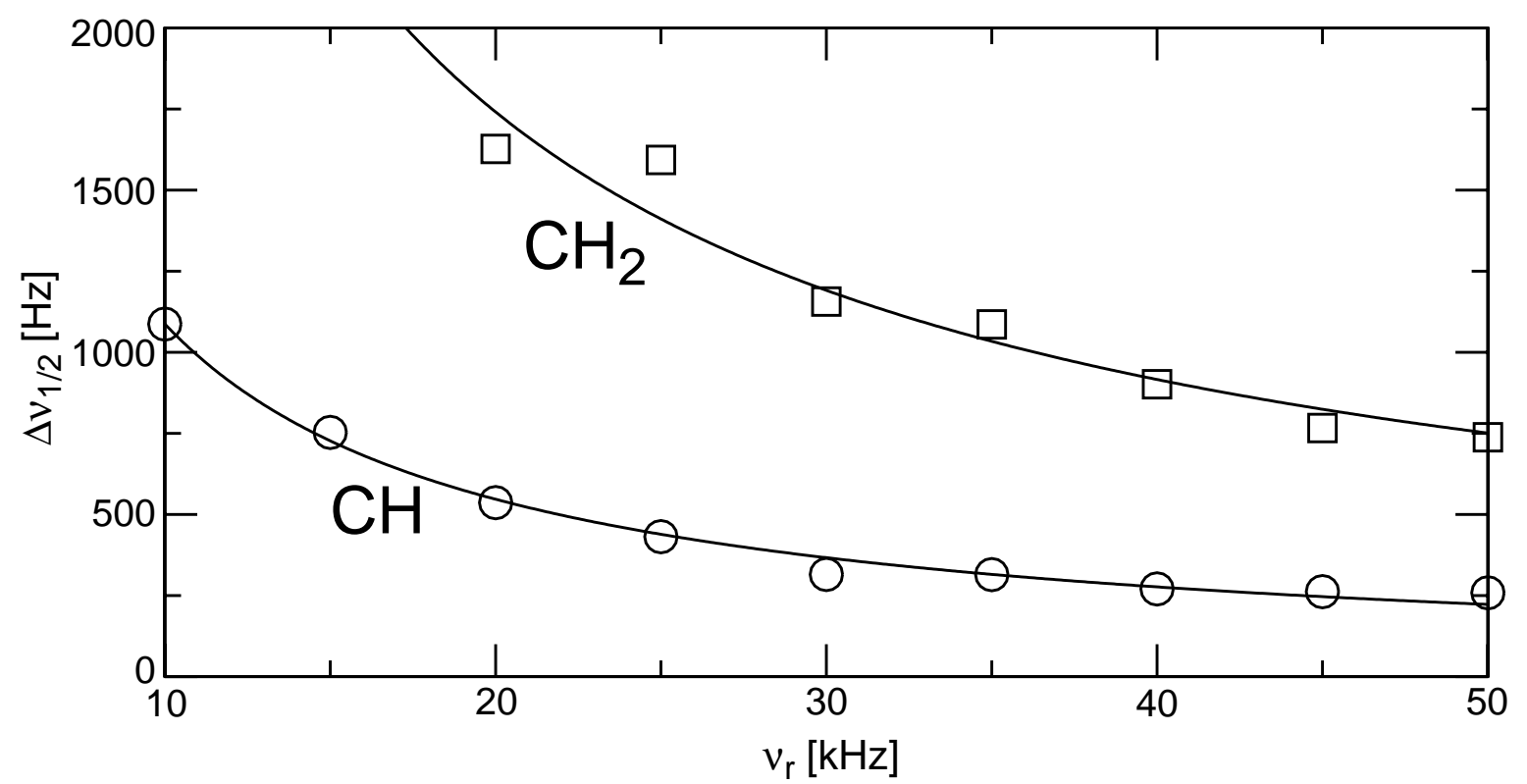

Figure 1.2: Dependence of the full width at half height (FWHH) on the spinning frequency for the spectra shown in Figure 1.1. The solid line shows the best fit of the function $\mathrm{f}\left(\mathrm{v}_{\mathrm{r}}\right)=\mathrm{a} / \mathrm{v}_{\mathrm{r}}+\mathrm{b}$ to the measured values. For the $\mathrm{CH}$ group $\mathrm{a}=10811 \mathrm{~Hz}^{2}, \mathrm{~b}=6.4 \mathrm{~Hz}$, for the $\mathrm{CH}_{2}$ group $\mathrm{a}=33002 \mathrm{~Hz}^{2}, \mathrm{~b}=91.0 \mathrm{~Hz}$. 
required that the decoupler field strength be significantly larger than two times the spinning frequency (see Chapter 4.3).

Due to the various interference effects between the sample spinning and the spin rotations by $\mathrm{rf}$ irradiation, it is difficult to imagine that efficient decoupling can be achieved if the time dependencies of the two processes are on the same scale. It seems, therefore, sensible to use rf-irradiation fields that either are considerably higher or considerably lower than the MAS spinning frequency. The much older and well-established approach is high-power decoupling using either cw irradiation or, more recently, multiple-pulse sequences. Under this condition, the spin Hamiltonian is first truncated by the rf irradiation and then on a longer time scale averaged by the MAS rotation (Figure 1.3a). In recent years, however, the available MAS spinning frequencies have increased quite substantially and have made the second approach also viable. If the MAS frequency is considerably larger than the rf-field amplitude, the Hamiltonian is first averaged by the MAS rotation and only this partially averaged Hamiltonian is then averaged by the rf field (Figure 1.3b).

a)

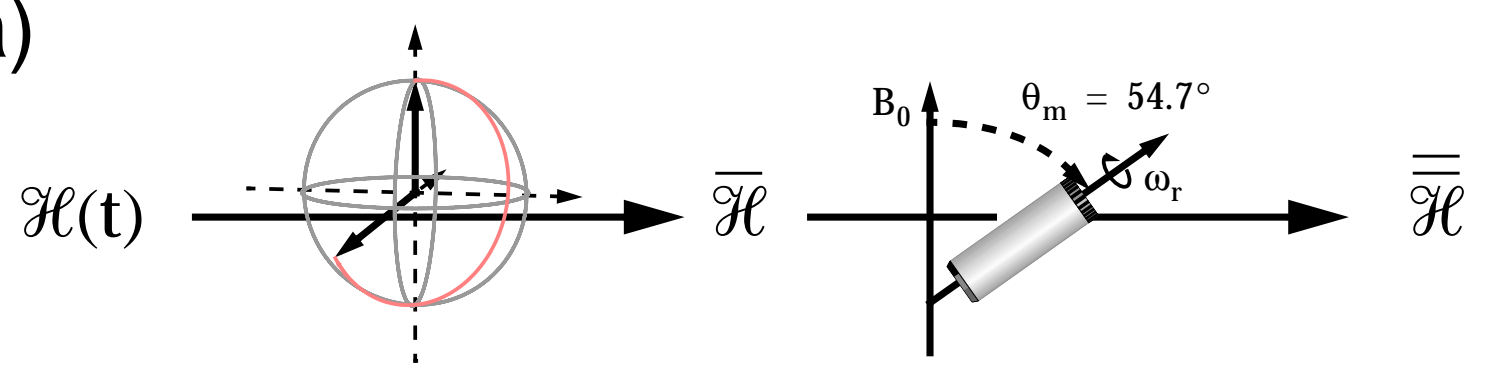

b)

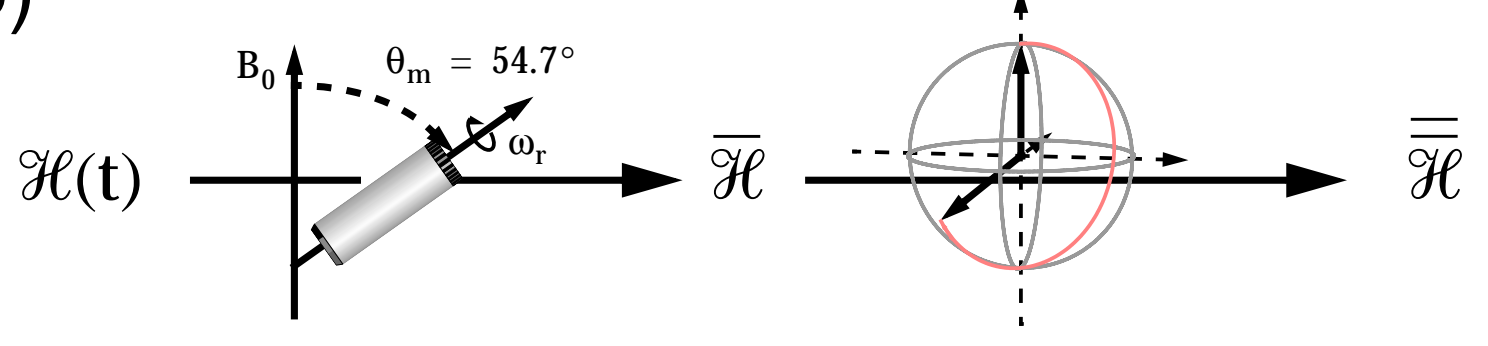

Figure 1.3: Sequential averaging of the Hamiltonian by space and spin rotations. In a) the Hamiltonian is first averaged in spin space and then in real space by MAS while in b) the Hamiltonian is first averaged by MAS and then in spin space. A prerequisite for such a treatment is that the two averaging processes happen on different time scales. 


\subsection{High-Power CW Decoupling}

For a long time, high-power cw irradiation was the most common way to achieve heteronuclear spin decoupling in solid-state NMR under MAS (17),(18),(19). The spins are irradiated with a "strong" rf-field of typically $50-250 \mathrm{kHz}$ for rigid organic solids, and the decoupling quality improves with increasing field strength. In this context, improved decoupling quality means narrower lines and higher line intensity. Figure 1.4 shows, as a typical example, the ${ }^{13} \mathrm{C}$ spectrum of the dipeptide LVal-L-Phe at an MAS frequency of $v_{\mathrm{r}}=28 \mathrm{kHz}$ without proton decoupling (Fig. 1.4a) and with high-power $\left(v_{1}=150 \mathrm{kHz}\right)$ proton decoupling (Fig. 1.4b). It is immediately obvious that the resolution of the spectrum is significantly improved by irradiating the protons. Such an observation is typical for a "normal" organic solid which has a strongly coupled proton network.

Despite the widespread use of cw decoupling under MAS in solid-state NMR, the source of the residual linewidth and the decoupling process in general were not well understood. There was quite a number of observations concerning the dependence of the line width on various parameters but no general theory which could explain all these observations. It was generally accepted that for efficient

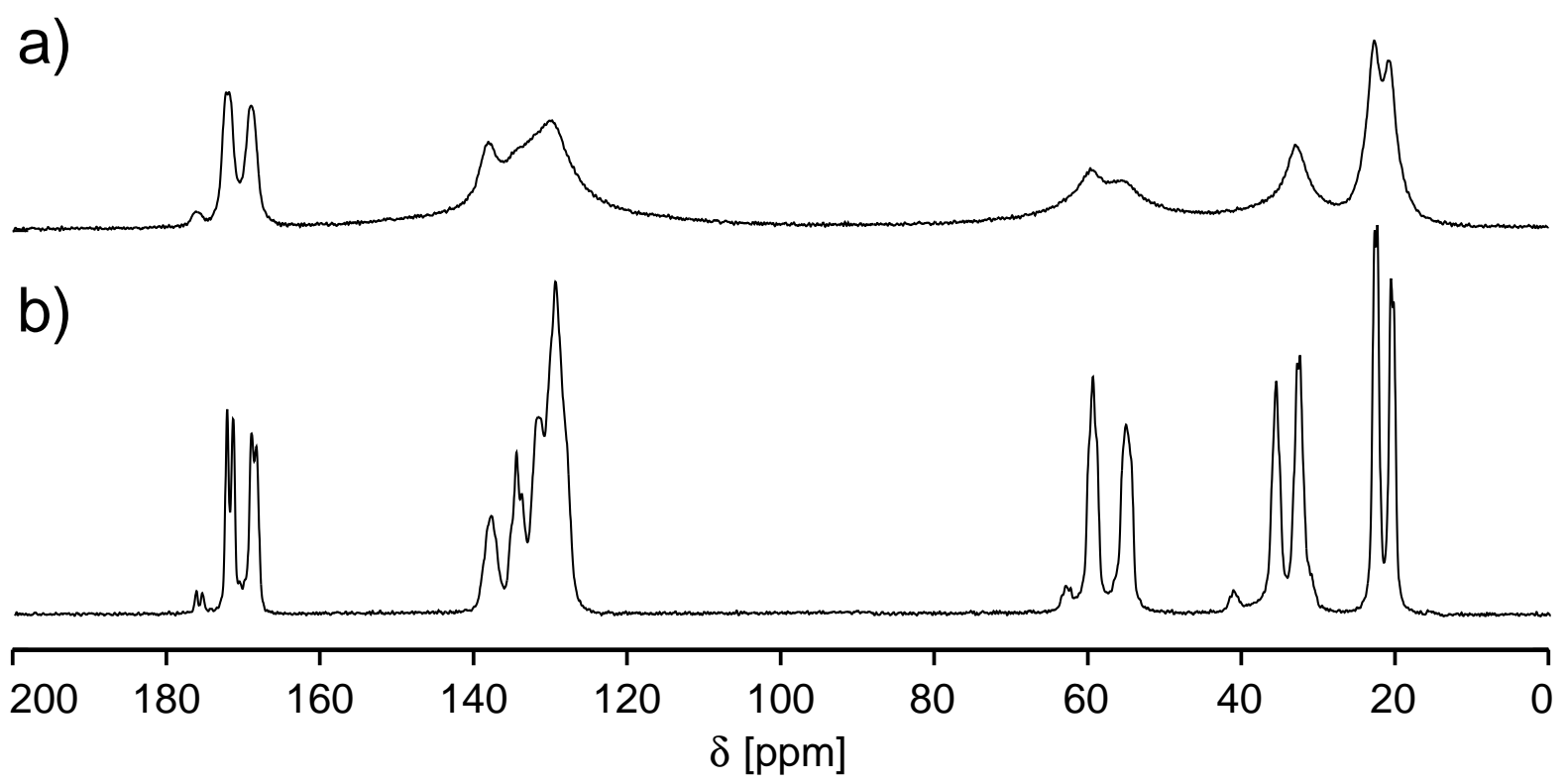

Figure 1.4: ${ }^{13} \mathrm{C}$ spectrum of a uniformly labeled dipeptide (L-Val-L-Phe) recorded without (a) and (b) with proton decoupling at an MAS frequency of $28 \mathrm{kHz}$. The increased resolution in the proton-decoupled spectrum is clearly visible. 
decoupling the field strength must be greater than the magnitude of both the heteronuclear and the homonuclear dipolar interactions. In solids with strongly coupled homonuclear spin systems the flip-flop fluctuations of the homonuclear spins (spin diffusion) lead to an additional modulation of the residual heteronuclear dipolar coupling which results in a narrowing of the observed line (21) due to an exchangetype process between the components of the multiplet (22) if the proton spin-diffusion rate constant is of the right order of magnitude. This line-narrowing process has also been called "self-decoupling" due to the proton spin-diffusion process (23),(24),(25). It has been shown experimentally that by applying the decoupling field off resonance such that the effective field is inclined at an angle of $54.74^{\circ}$ (magic angle) to the static magnetic field, the homonuclear interactions are quenched. The reduced or vanishing homonuclear dipolar interactions lead to a broadening of the decoupled heteronuclear line (18). The residual linewidth for $\mathrm{cW}$ decoupling in solids under MAS is usually found to decrease with increasing rf-field strength (26). It has also been observed that the residual linewidth increases with increasing MAS frequency if the rf-field strength is kept constant (27). Decoupling side bands can be observed at the rf-irradiation frequency (28). A detailed discussion of the various contributions to the line width of solid-state NMR spectra under high-power cw decoupling and MAS is found in the literature (29).

However, the models presented so far can not explain the two experimental ${ }^{15} \mathrm{~N}$ spectra of $\left[\mathrm{d}_{9}\right]$-trimethyl-ammonium chloride shown in Figure 1.5. Without proton decoupling (Fig 1.5a) the spectrum shows two sharp lines (FWHH $\Delta_{1 / 2} \approx$ $50 \mathrm{~Hz}$ ) with a splitting due to the J-coupling $\left({ }^{1} \mathrm{~J}_{\mathrm{NH}} \approx 100 \mathrm{~Hz}\right)$. This spectrum was measured at an spinning frequency of $30 \mathrm{kHz}$ and at a proton resonance frequency of $600 \mathrm{MHz}$. Under cw decoupling with a field strength of $v_{1}=100 \mathrm{kHz}$ (Fig. $1.5 \mathrm{~b}$ ) the line broadens significantly and one finds two broad resonances with a splitting of about $850 \mathrm{~Hz}$. This unexpected experimental observation of a line broadening instead a line narrowing under cw decoupling made it clear that a new model was needed to understand the mechanism of heteronuclear spin decoupling under MAS in solids.

The different behavior of the two samples (Figs. 1.4 and 1.5) under high-power cw decoupling can be understood within a model of decoupling which explicitly 
includes the chemical-shielding tensor of the protons and the proton spin-diffusion process (21),(30). This model is based on the coherent description of a two-spin system which is coupled to the proton bath by an isotropic relaxation-type superoperator. The system Hamiltonian of the two-spin system is first averaged by the rf-field and then in a second step by the MAS rotation (Figure 1.3a). Such an approach is justified if the MAS rotation is considerably (at least a factor of three) slower than the rf-nutation frequency (the "rf-field strength"). The truncation of the two-spin Hamiltonian by the rf field leads to a second-order cross term between the chemical-shielding tensor of the protons and the heteronuclear dipolar coupling (30). This term, mentioned in passing in Ref. (20), is in many cases the dominant contribution to the residual line width of an isolated two-spin system under $\mathrm{cw}$ decoupling. This conclusion was later confirmed in a paper by Zax (31) where the possible mechanisms for the residual line broadening in $\mathrm{cw}$ decoupling were discussed.

Such a second-order cross term cannot be averaged out by the MAS rotation since the coupling of the two tensors leads to a sum of a zeroth-rank, a second-rank, and a fourth-rank tensor. Only the second-rank tensor contribution is averaged out by

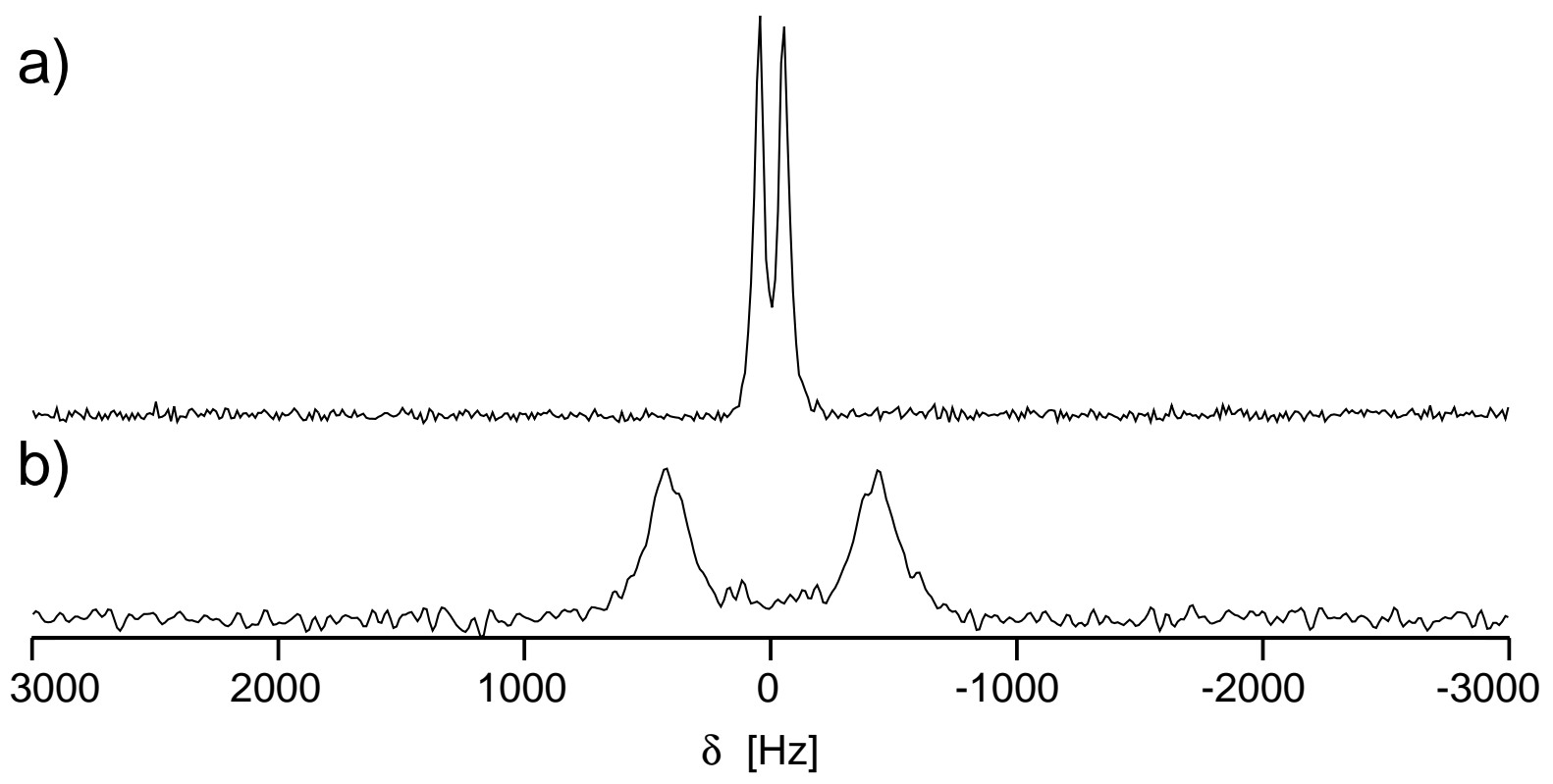

Figure 1.5: ${ }^{15} \mathrm{~N}$ spectrum of $\left[\mathrm{d}_{9}\right]$-trimethyl-ammonium chloride (a) without and (b) with decoupling. In this sample we see a significant broadening of the resonances as a result of proton decoupling. 
the MAS rotation. However, by using different spatial averaging techniques such as dynamic-angle spinning (DAS) or double rotation (DOR) (32), (33), (34) it would be possible to average out both the second-rank and the fourth-rank parts of the cross term simultaneously, leaving only the isotropic (zeroth-rank tensor) part unchanged.

Second-order effects in the laboratory frame due to the truncation of the Hamiltonian by the Zeeman field are well known in solid-state NMR (17),(18),(35). The best known example is the second-order quadrupolar shift (36) but other examples such as a shift originating from the dipolar coupling have been described and observed experimentally (37),(38). There is also an isotropic second-order dipolar shift in the rotating frame (39) which can be substantially larger than the second-order dipolar shifts in the laboratory frame (37),(38) because the interaction is scaled by the rf field and not by the Zeeman field. The second-order dipolar shift in the rotating frame is very closely related to the second-order cross term of the dipolar coupling and the chemical-shielding tensors. However, since the Hamiltonian describing the second-order dipolar shift commutes with the S-spin subspace of the Hamiltonian, it has no direct influence on the spectrum of the observed spin.

\subsection{High-Power Multiple-Pulse Decoupling}

The two-pulse phase-modulated (TPPM) decoupling sequence (20) was the first multiple-pulse decoupling method for solid organic samples under MAS that gave significant improvements in both line width and line intensity under a wide range of experimental conditions. TPPM decoupling consists of two pulses each with a flip angle of about $180^{\circ}$ and a phase difference between the two pulses, $\varphi$, which is on the order of $10^{\circ}$ to $50^{\circ}$. It was shown that the significant reduction in the residual line width comes from the fact that the second-order cross term between the chemicalshielding tensor of the irradiated spins and the heteronuclear dipolar-coupling tensor is reduced by TPPM decoupling (30),(40),(41). Experimentally, it was found that the line width in TPPM decoupling is very sensitive to the precise setting of the two parameters, the pulse length and the phase angle (21). They have to be optimized 
empirically after any change in experimental parameters and depend on the rf-field strength, MAS frequency, sample, spectrometer, and probe.

There are a number of variations and modifications of the TPPM decoupling sequence. Frequency-modulated and phase-modulated decoupling (FMPM) (42) combines the phase modulation with a frequency modulation leading to a circular modulation of the rf irradiation. Only one of the two possible circular modulations works as well as TPPM while the other one does not give any significant improvement compared to $\mathrm{cw}$ decoupling. The small phase angle rapid cycling (SPARC) sequences (43) and the small phase incremental alternation (SPINAL) sequences (44) combine the TPPM scheme with a phase supercycle. They show significant improvements in the offset behavior compared to TPPM in liquidcrystalline samples. However, in rigid, powdered solids the SPARC and SPINAL sequences do not perform much better than the original TPPM sequence. An amplitude-modulated variation of TPPM called AM-TPPM (41) uses third averaging by adding an amplitude modulated field to the cw component of the TPPM irradiation. Again, this modification shows very little improvement compared to the original TPPM sequence.

A different type of rotor-synchronized decoupling sequence, $\mathrm{C}_{12}^{-1}$ (45), is based on a series of phase-shifted $(2 \pi)_{\varphi}$ pulses and requires that the rf-field amplitude be equal to six times the spinning frequency. The phase, $\varphi$, is shifted by $30^{\circ}$ between successive pulses. The sequence's decoupling performance is comparable to that obtained by TPPM decoupling, but the rotor synchronization and the requirement of an rf-field amplitude of six times the spinning frequency make this sequence less desirable at higher MAS frequencies.

Another type of rotor-synchronized decoupling sequence called $X$-inverse- $X$ (XiX) (46) consists of two pulses of equal length with a phase difference of $180^{\circ}$. The decoupling performance depends only on the length of the pulse and not on the flip angle of the pulse, i.e., not on the rf-field amplitude. Under many experimental conditions, XiX decoupling leads to significant improvements in line intensity compared to TPPM decoupling. In addition, the XiX decoupling sequence has only 
one adjustable parameter, namely the pulse length, and its optimum value is quite well defined by the spinning frequency. Therefore, one only needs to perform a local one-parameter optimization compared to a two-dimensional optimization (pulse length and pulse phase) in the case of the TPPM sequence. In addition, the XiX sequence is less sensitive to rf-field inhomogeneities than the TPPM decoupling sequence.

A common characteristic of all these sequences is that the best decoupling is typically achieved for the highest possible rf-field amplitude after optimization of all the remaining parameters.

\subsection{Low-Power Decoupling}

Obviously, it is desirable to achieve the best possible decoupling with the lowest possible rf-field amplitude. Irradiating a sample using high-power rf fields for extended periods of time can lead to undesired heating of the sample especially if the sample contains water. In addition, high rf fields put considerable strain on the electronic components in the probe circuit and limit the acquisition time.

Over the past ten years the maximum obtainable MAS frequencies have increased significantly. Today, commercially available $2.5 \mathrm{~mm}$ o.d. rotors allow spinning frequencies up to $30 \mathrm{kHz}$ routinely, and spinning frequencies up to $50 \mathrm{kHz}$ can be obtained safely using experimental $1.8 \mathrm{~mm}$ o.d. spinning systems (47). As mentioned above, high MAS frequencies require, for high-power decoupling schemes, correspondingly higher decoupling fields. At an MAS frequency of $50 \mathrm{kHz}$ the minimum required rf-field strength for effective decoupling is about $150 \mathrm{kHz}$.

The demand for higher MAS frequencies is driven by several developments. Firstly, increasing $\mathrm{B}_{0}$-field strengths lead to an increase in the size of the CSA tensors. To obtain a spectrum without strong sidebands one needs to spin faster than the size of the CSA tensor, which is on the order of $30 \mathrm{kHz}$ for a carbonyl carbon in a $\mathrm{B}_{0}$ field of $18.8 \mathrm{~T}$ ( $800 \mathrm{MHz}$ proton resonance frequency). Secondly, the increase in $\mathrm{B}_{0}$ fields also leads to a larger spread of the isotropic chemical shifts. To avoid rotational- 
resonance recoupling conditions for uniformly labeled samples, it is best to spin faster than the width of the spectrum. For an 18.8-T magnet this corresponds to a spinning frequency of $35 \mathrm{kHz}$ for a typical ${ }^{13} \mathrm{C}$ spectrum. Lastly, it has been observed experimentally that the linewidth in uniformly ${ }^{13} \mathrm{C}$-labeled compounds decreases with increasing spinning frequency.

As an alternative to high-power decoupling one can use low-power cw decoupling (48) in addition to fast MAS in order to achieve heteronuclear spin decoupling with minimum power dissipation in the sample. Such an approach is based on the reversal of the averaging processes compared to high-power decoupling (Figure 1.3b). First the Hamiltonian is averaged by the MAS rotation, and the timeaveraged Hamiltonian is then truncated, in a second step, by a weak rf field. The main requirement for such a scheme is that the terms in the time-averaged Hamiltonian are significantly smaller than the applied rf field. The maximum size of the rf-field is determined by the fact that recoupling conditions $(12),(13),(14),(15),(16)$ have to be avoided since they can lead to significant broadening of the lines by reintroduction of the dipolar coupling.

\subsection{General Theoretical Framework}

For the theoretical description of decoupling in typical organic solids, we have to consider a system composed of a single $S$ spin and many I spins. This corresponds to the situation of a dilute rare spin such as ${ }^{13} \mathrm{C}$ or ${ }^{15} \mathrm{~N}$ coupled to an abundant spin bath such as that given by ${ }^{1} \mathrm{H}$. The time-dependent Hamiltonian under MAS of such a system in the usual rotating frame, i.e., rotating with the Zeeman frequencies of the nuclei about the $\mathrm{B}_{0}$-field direction, is given by

$$
\begin{aligned}
\mathscr{H}(\mathrm{t})= & \mathscr{H}_{\mathrm{S}}^{\mathrm{CS}}+\mathscr{H}_{\mathrm{I}}^{\mathrm{CS}}+\mathscr{H}_{\mathrm{II}}^{\mathrm{J}}+\mathscr{H}_{\mathrm{SI}}^{\mathrm{J}}+ \\
& \mathscr{H}_{\mathrm{S}}^{\mathrm{CSA}}(\mathrm{t})+\mathscr{H}_{\mathrm{I}}^{\mathrm{CSA}}(\mathrm{t})+\mathscr{H}_{\mathrm{II}}^{\mathrm{dip}}(\mathrm{t})+\mathscr{H}_{\mathrm{SI}}^{\mathrm{dip}}(\mathrm{t})+ \\
& \mathscr{H}_{\mathrm{I}}^{\mathrm{rf}}(\mathrm{t})
\end{aligned}
$$

assuming that the I spins and the $S$ spin have a spin-quantum number of $1 / 2$. The first four terms of Eq. [1.1] are time-independent; $\mathscr{H}_{\mathrm{S}}^{\mathrm{CS}}$ and $\mathscr{H}_{\mathrm{I}}^{\mathrm{CS}}$ are the isotropic 
chemical shifts of spins $\mathrm{S}$ and I, respectively; $\mathscr{H}_{\mathrm{II}}^{\mathrm{J}}$ describes the homonuclear isotropic J-couplings among the I spins; and $\mathscr{H}_{\mathrm{SI}}^{\mathrm{J}}$ describes the heteronuclear isotropic J-couplings between the S spin and the I spins. The last four terms of Eq. [1.1] describe orientation-dependent second-rank tensor quantities, which are time dependent due to the mechanical rotation of the sample about the magic angle. The chemical-shielding tensors of spins $\mathrm{S}$ and I are described by $\mathscr{H}_{\mathrm{S}}^{\mathrm{CSA}}(\mathrm{t})$ and $\mathscr{H}_{\mathrm{I}}^{\mathrm{CSA}}(\mathrm{t})$, respectively, while $\mathscr{H}_{\mathrm{II}}^{\mathrm{dip}}(\mathrm{t})$ describes the homonuclear dipolar couplings among the I spins, and $\mathscr{H}_{\mathrm{SI}}^{\mathrm{dip}}(\mathrm{t})$ describes the heteronuclear dipolar couplings between the $\mathrm{S}$ spin and the I spins. The time-dependent rf irradiation (if any) on the I spins is described by $\mathscr{H}_{\mathrm{I}}^{\mathrm{rf}}(\mathrm{t})$. Explicit expressions for the different terms can be found in the literature $(17),(18),(19)$ and are given in Appendix A.

In addition to the time dependence due to the MAS rotation, all second-rank tensor terms also depend on the orientation of the crystallite in the rotor-fixed coordinate system ("powder average"). In principle, one should write all the timedependent Hamiltonians of Eq. [1.1] not only as a function of time but also as a function of the crystallite orientation, e.g., $\mathscr{H}(\mathrm{t}, \Omega)$, where $\Omega=(\alpha, \beta, \gamma)$ is the set of three Euler angles which describes the orientation of the principal-axes system of the interaction in the rotor-fixed frame of reference. For simplicity of notation this dependence on the Euler angles will be omitted and only explicitly written where it is important for the understanding of a given concept. To obtain the full spectrum of a powder sample, we have to average the time-domain signal $\mathscr{S}(\mathrm{t}, \alpha, \beta, \gamma)$ over all possible orientations of the crystallites

$$
\mathscr{Y}(\mathrm{t})=\frac{1}{8 \pi^{2}} \int_{0}^{2 \pi} \mathrm{d} \alpha \int_{0}^{\pi} \sin \beta \mathrm{d} \beta \int_{0}^{2 \pi} \mathrm{d} \gamma \cdot \mathscr{Y}(\mathrm{t}, \alpha, \beta, \gamma)
$$

To analyze a Hamiltonian with multiple time-dependencies such as the one of Eq. [1.1] analytically or numerically, there are different strategies available. One can either use Shirley's Floquet approach (49),(50) to convert the time-dependent Hamiltonian into a time-independent but infinite-dimensional Hamiltonian. If the two time dependencies are incommensurate, one has to use a bimodal Floquet approach (51),(52),(53),(54). This Floquet Hamiltonian can then either be diagonalized 
analytically or numerically, or one can apply static perturbation theory to obtain physical insight into the problem. If the two time dependencies are commensurate one can also apply average Hamiltonian theory (AHT) (1),(17) to obtain a timeindependent effective Hamiltonian. For special classes of multiple-pulse sequences under MAS, one can use symmetry rules $(40),(45)$ to simplify the calculation of the zeroth-order average Hamiltonian and to estimate the size of the first-order terms. If the time scales of the two time-dependent processes are very different, one can also apply average Hamiltonian theory using a sequential averaging approach. This approach assumes that on the time scale of the faster averaging process, the slower time dependence is quasi-static and can be neglected. Such an approach has to be applied carefully since it is not able to describe any interference effects between the two time-dependent processes. Several of these methods will be used in the following chapters to analyze the decoupling problem under various conditions.

\subsection{References}

1. R.R. Ernst, G. Bodenhausen, and A. Wokaun, Principles of Nuclear Magnetic Resonance in One and Two Dimensions. Clarendon Press, Oxford, 1987.

2. H.J. Reich, M. Jautelat, M.T. Messe, F.J. Weigert, and J.D. Roberts, J. Am. Chem. Soc. 91, 7445 (1969).

3. B. Birdsall, N.J.M. Birdsall, and J Feeney, J. Chem. Soc. Chem. Commun. 1972, 316 (1972).

4. R.R. Ernst, J. Chem. Phys. 45, 3845 (1966).

5. M.H. Levitt, R. Freeman, and T. Frenkiel, Advances in Magnetic Resonance, volume 11. Academic Press, New York, 1982.

6. A.J. Shaka, J. Keeler, T. Frenkiel, and R. Freeman, J. Magn. Reson. 52, 335-338 (1983).

7. A.J. Shaka, J. Keeler, and R. Freeman, J. Magn. Reson. 53, 313 (1983).

8. A.J. Shaka, C.J. Lee, and A. Pines, J. Magn. Reson. 77, 274 (1988). 
9. A.J. Shaka, P.B. Barker, and R. Freeman, J. Magn. Reson. 64, 547 (1985).

10. E. Kupce and R. Freeman, J. Magn. Reson. Ser. A 115, 273-276 (1995).

11. H. Geen and J.M. Bohlen, J. Magn. Reson. 125, 376-382 (1997).

12. D.P. Raleigh, A.C. Kolbert, T.G. Oas, M.H. Levitt, and R.G. Griffin, J.Chem.Soc.,Faraday Trans. 84, 3691 (1988).

13. T.G. Oas, R.G. Griffin, and M.H. Levitt, J. Chem. Phys. 89, 692 (1988).

14. M.H. Levitt, T.G. Oas, and R.G. Griffin, Isr. J. Chem. 28, 271-282 (1988).

15. A.C. Kolbert, D.P. Raleigh, R.G. Griffin, and M.H. Levitt, J. Magn. Reson. 89, 133 (1990).

16. N.C. Nielsen, H. Bildsoe, H.J. Jakobsen, and M.H. Levitt, J. Chem. Phys. 101, 1805 (1994).

17. U. Haeberlen, High Resolution NMR in Solids: Selective Averaging. Academic Press New York, 1976.

18. M. Mehring, Principles of High Resolution NMR in Solids, 2nd edition. Springer, Berlin, 1983.

19. K.Schmidt-Rohr and H.W. Spiess, Multidimensional Solid-State NMR and Polymers. Academic Press, London, 1994.

20. A.E. Bennett, C.M. Rienstra, M. Auger, K.V. Lakshmi, and R.G. Griffin, J. Chem. Phys. 103, 6951-6958 (1995).

21. M. Ernst, H. Zimmermann, and B.H. Meier, Chem. Phys. Lett. 317, 581-588 (2000).

22. M. Ernst, A Verhoeven, and B.H. Meier, J. Magn. Reson. 130, 176-185 (1998).

23. M. Mehring, G. Sinning, and A. Pines, Z. Phys. B 24, 73 (1976).

24. G. Sinning, M. Mehring, and A. Pines, Chem. Phys. Lett. 43, 382-386 (1976).

25. M. Mehring and G. Sinning, Phys. Rev. B: Condens. Matter 15, 2519-2532 (1977).

26. P. Tekely, P. Palmas, and D. Canet, J. Magn. Reson. A 107, 129-133 (1994).

27. I.J. Shannon, K.D.M. Harris, and S. Arumugan, Chem.Phys.Lett. 196, 588-594 (1992). 
28. J.R. Sachleben, S. Caldarelli, and L. Emsley, J. Chem. Phys. 104, 2518 (1996).

29. D.L. VanderHart, W.L. Earl, and A.N. Garroway, J. Magn. Reson. 44, 361-401 (1981).

30. M. Ernst, S. Bush, A.C. Kolbert, and A. Pines, J. Chem. Phys. 105, 3387-3397 (1996).

31. D.B. Zax, J. Chem. Phys. 105, 6616-6625 (1996).

32. K.T. Mueller, B.Q. Sun, G.C. Chingas, J.W. Zwanziger, T. Terao, and A. Pines, J. Magn. Reson. 86, 470 (1990).

33. A. Llor and J. Virlet, Chem.Phys.Lett. 152, 248-253 (1988).

34. A. Samoson, E. Lippmaa, and A. Pines, Mol.Phys. 65, 1013 (1988).

35. A. Abragam, The Principles of Nuclear Magnetism. Clarendon Press, Oxford, 1961.

36. D. Freude and J. Haase, Quadrupole Effects in Solid-State Magnetic Resonance, volume 29, Springer-Verlag , 1993.

37. D.L. VanderHart, J. Chem. Phys. 84, 1196 (1986).

38. M.P. Augustine, K.W. Zilm, and D.B. Zax, J. Chem. Phys. 98, 9432 (1993).

39. M. Ernst, A.C. Kolbert, K. Schmidtrohr, and A. Pines, J. Chem. Phys. 104, 82588268 (1996).

40. M. Carravetta, M. Eden, X. Zhao, A. Brinkmann, and M. H. Levitt, Chem. Phys. Lett. 321, 205-215 (2000).

41. K. Takegoshi, J. Mizokami, and T. Terao, Chem. Phys. Lett. 341, 540-544 (2001).

42. Z.H. Gan and R.R. Ernst, Solid State NMR 8, 153-159 (1997).

43. Youlu Yu and B. M. Fung, J. Magn. Reson. 130, 317-320 (1998).

44. B.M. Fung, A.K. Khitrin, and K. Ermolaev, J. Magn. Reson. 142, 97-101 (2000).

45. M. Eden and M. H. Levitt, J. Chem. Phys. 111, 1511-1519 (1999).

46. A. Detken, E. Hardy, M. Ernst, and B.H. Meier, Chem. Phys. Lett. submitted, (2002).

47. A. Samoson, T. Tuherm, and J. Past, J. Magn. Reson. 149, 264-267 (2001). 
48. M. Ernst, A. Samoson, and B.H. Meier, Chem. Phys. Lett. 348, 293-302 (2001).

49. J.H. Shirley, Phys.Rev. 138B, 979 (1965).

50. S.-I. Chu, Adv.Chem.Phys. 73, 739 (1989).

51. M. Baldus, T. O. Levante, and B.H. Meier, Z. Naturforsch. 49a, 80-88 (1994).

52. T. O. Levante, M. Baldus, B. H. Meier, and R. R. Ernst, Mol. Phys. 86, 1195-1212 (1995).

53. E. Vinogradov, P.K. Madhu, and S. Vega, Chem. Phys. Lett. 329, 207-214 (2000).

54. E. Vinogradov, P.K. Madhu, and S. Vega, J. Chem. Phys. 115, 8983-9000 (2001). 


\section{High-Power CW Decoupling in Isolated Spin Systems}

\subsection{Theory}

The general case of a single S spin coupled to many homonuclearly coupled I spins (see Eq. [1.1]) is quite complicated and cannot usually be treated analytically. We, therefore, simplify the system for our calculations and consider only the case of a heteronuclear two-spin system under $\mathrm{cw}$ rf irradiation. This corresponds to a $\mathrm{C}-\mathrm{H}$ or $\mathrm{N}-\mathrm{H}$ system where we neglect the long-range heteronuclear dipolar couplings as well as all the homonuclear dipolar and J couplings among the protons. The Hamiltonian for such a system is given by a simplified version of Eq. [1.1] which contains only the following terms:

$$
\begin{aligned}
\mathscr{H}(\mathrm{t})= & \mathscr{H}_{\mathrm{S}}^{\mathrm{CS}}+\mathscr{H}_{\mathrm{I}}^{\mathrm{CS}}+\mathscr{H}_{\mathrm{SI}}^{\mathrm{J}}+\mathscr{H}_{\mathrm{S}}^{\mathrm{CSA}}(\mathrm{t})+\mathcal{H}_{\mathrm{I}}^{\mathrm{CSA}}(\mathrm{t})+\mathcal{H}_{\mathrm{SI}}^{\mathrm{dip}}(\mathrm{t})+\mathscr{H}_{\mathrm{I}}^{\mathrm{rf}} \\
= & \omega_{\mathrm{S}}^{\text {iso }} \cdot \mathrm{S}_{\mathrm{z}}+\omega_{\mathrm{I}}^{\text {iso }} \cdot \mathrm{I}_{\mathrm{z}}+\pi \mathrm{J} \cdot 2 \mathrm{~S}_{\mathrm{z}} \mathrm{I}_{\mathrm{z}} \\
& +\omega_{\mathrm{S}}(\mathrm{t}) \cdot \mathrm{S}_{\mathrm{z}}+\omega_{\mathrm{I}}(\mathrm{t}) \cdot \mathrm{I}_{\mathrm{z}}+\omega_{\mathrm{SI}}(\mathrm{t}) \cdot 2 \mathrm{~S}_{\mathrm{z}} \mathrm{I}_{\mathrm{z}} \\
& +\omega_{1} \cdot \mathrm{I}_{\mathrm{x}}
\end{aligned}
$$

High-power cw decoupling describes the situation where the nutation frequency corresponding to the amplitude of the rf field, $\omega_{1}$, is considerably larger than the frequency of the MAS rotation, $\omega_{\mathrm{r}}$. In this case, we can assume that the averaging by the rf field occurs on a much shorter time scale than the averaging by the MAS sample rotation. We can, therefore, use a sequential averaging approach (see Figure 1.3a) where we first calculate the effect of the rf-field on the quasi-static system Hamiltonian and then, in a second step, determine the influence of the MAS rotation on this partially averaged Hamiltonian. There are several ways to do this calculation. One possibility is to transform the system Hamiltonian into an interaction frame quantized along the rf field and apply average Hamiltonian theory to obtain a timeindependent, time-averaged Hamiltonian. We will, however, use a different approach here. For a two-spin system we can analytically diagonalize the Hamiltonian under the assumption that it is quasi-static on the time-scale of the rf-nutation frequency. The quasi-static analytical solution can then be expanded in a power series. Finally, 
the influence of the MAS rotation on the eigenvalues of the quasi-static diagonalized Hamiltonian is calculated.

Such an sequential approach will be compared with an approach where the averaging by the rf-field and by the MAS rotation are treated simultaneously using average Hamiltonian theory. Here, we have to assume that the rf-field amplitude is an integer multiple of the MAS frequency.

\subsubsection{Sequential Averaging Approach}

We can diagonalize the Hamiltonian of Eq. [2.1] analytically and obtain four eigenvalues. For an initial density operator $\sigma(0)=S_{x}$ and a phase-sensitive detection operator $\mathrm{S}^{-}$we obtain an FID

$$
\mathscr{S}\left(\mathrm{t}, \mathrm{t}_{2}\right)=\sum_{\mathrm{m}=1}^{4} \mathscr{Y}_{\mathrm{m}}(\mathrm{t}) \cdot \mathrm{e}^{+\mathrm{i} \cdot \omega^{(\mathrm{m})}(\mathrm{t}) \cdot \mathrm{t}_{2}}
$$

which has four components. The four transition frequencies $\omega^{(\mathrm{m})}(\mathrm{t})(\mathrm{m}=1 \ldots 4)$ are symmetric about the carbon resonance frequency, $\omega_{S}^{\text {iso }}+\omega_{S}(t)$, and are given by (1):

$$
\begin{aligned}
& \omega^{(1,2)}(\mathrm{t})=\omega_{\mathrm{S}}^{\text {iso }}+\omega_{\mathrm{S}}(\mathrm{t}) \pm \frac{\omega_{1}}{2} \cdot\left[\sqrt{1+\left(\frac{\left(\omega_{\mathrm{SI}}(\mathrm{t})+\pi \mathrm{J}_{\mathrm{SI}}\right)+\left(\omega_{\mathrm{I}}(\mathrm{t})+\omega_{\mathrm{I}}^{\text {iso }}\right)}{\omega_{1}}\right)^{2}}\right. \\
&\left.+\sqrt{1+\left(\frac{\left(\omega_{\mathrm{SI}}(\mathrm{t})+\pi \mathrm{J}_{\mathrm{SI}}\right)-\left(\omega_{\mathrm{I}}(\mathrm{t})+\omega_{\mathrm{I}}^{\text {iso }}\right)}{\omega_{1}}\right)^{2}}\right]
\end{aligned}
$$

and

$$
\begin{aligned}
\omega^{(3,4)}(t)=\omega_{S}^{\text {iso }}+\omega_{S}(t) \pm \frac{\omega_{1}}{2} & \cdot\left[\sqrt{1+\left(\frac{\left(\omega_{S I}(t)+\pi J_{S I}\right)+\left(\omega_{I}(t)+\omega_{I}^{\text {iso }}\right)}{\omega_{1}}\right)^{2}}\right. \\
& \left.-\sqrt{1+\left(\frac{\left(\omega_{S I}(t)+\pi J_{S I}\right)-\left(\omega_{I}(t)+\omega_{I}^{\text {iso }}\right)}{\omega_{1}}\right)^{2}}\right]
\end{aligned}
$$


All symbols used in the Eqs. [2.3] and [2.4] are defined in Appendix A. For the limit of no decoupling $\left(\omega_{1}=0 \mathrm{~Hz}\right)$, we obtain the double-quantum and zero-quantum transitions

$$
\omega^{(1,2)}(t)=\left(\omega_{S}^{\text {iso }}+\omega_{S}(t)\right) \pm\left(\omega_{I}^{\text {iso }}+\omega_{I}(t)\right)
$$

of the SI two-spin system and the two one-quantum transitions

$$
\omega^{(3,4)}(\mathrm{t})=\left(\omega_{S}^{\text {iso }}+\omega_{S}(t)\right) \pm\left(\omega_{S I}(t)+\pi J_{S I}\right)
$$

of the S spin. The corresponding signal intensities of these four resonance lines are given by

$$
\mathscr{S}_{1,2}(\mathrm{t})=\frac{1}{4}-\frac{1}{4}\left(1+\frac{4\left(\omega_{\mathrm{SI}}(\mathrm{t})+\pi \mathrm{J}_{\mathrm{SI}}\right)^{2} \omega_{1}^{2}}{\left(\left(\omega_{\mathrm{SI}}(\mathrm{t})+\pi \mathrm{J}_{\mathrm{SI}}\right)^{2}+\left(\omega_{\mathrm{I}}(\mathrm{t})+\omega_{\mathrm{I}}^{\text {iso }}\right)^{2}+\omega_{1}^{2}\right)^{2}}\right)^{-1 / 2}
$$

and

$$
\mathscr{Y}_{3,4}(\mathrm{t})=\frac{1}{4}+\frac{1}{4}\left(1+\frac{4\left(\omega_{\mathrm{SI}}(\mathrm{t})+\pi \mathrm{J}_{\mathrm{SI}}\right)^{2} \omega_{1}^{2}}{\left(\left(\omega_{\mathrm{SI}}(\mathrm{t})+\pi \mathrm{J}_{\mathrm{SI}}\right)^{2}+\left(\omega_{\mathrm{I}}(\mathrm{t})+\omega_{\mathrm{I}}^{\text {iso }}\right)^{2}+\omega_{1}^{2}\right)^{2}}\right)^{-1 / 2}
$$

In the limit of strong decoupling, i.e., $\omega_{1} »\left(\omega_{\mathrm{SI}}(\mathrm{t})+\pi \mathrm{J}_{\mathrm{SI}}\right)$ and $\omega_{1} »\left(\omega_{\mathrm{I}}(\mathrm{t})+\omega_{\mathrm{I}}^{\text {iso }}\right)$, the intensities $\mathscr{S}_{1}(\mathrm{t})$ and $\mathscr{S}_{2}(\mathrm{t})$ are small. The transition frequencies $\omega^{(1)}(\mathrm{t})$ and $\omega^{(2)}(\mathrm{t})$ (Eq. [2.3]) correspond to the decoupling sidebands, which appear with a frequency offset approximately equal to the rf-field strength (2). They will, therefore, be neglected in the further discussion. Without $\mathrm{rf}$ irradiation, i.e., $\omega_{1}=0 \mathrm{~Hz}$, the intensities $\mathscr{S}_{1}(\mathrm{t})$ and $\mathscr{T}_{2}(\mathrm{t})$ are, as expected, zero. The intensities $\mathscr{S}_{3}(\mathrm{t})$ and $\mathscr{T}_{4}(\mathrm{t})$ tend towards $1 / 2$ for strong and vanishing decoupling fields.

To analyze the coupling between the heteronuclear coupling term, $\omega_{S I}(t)+\pi J_{S I}$, and the chemical-shift term, $\omega_{I}(t)+\omega_{I}^{\text {iso }}$, in Eq. [2.4], we expand the square root as a power series. Assuming that the decoupling field strength is much larger than the dipolar-coupling or the chemical-shielding tensors (strong decoupling limit) we obtain 


$$
\begin{aligned}
\omega^{(3,4)}(\mathrm{t})=\omega_{\mathrm{S}}^{\text {iso }}+\omega_{\mathrm{S}}(\mathrm{t}) \pm \frac{\omega_{1}}{2} \cdot & {\left[1+\frac{1}{2} \cdot\left(\frac{\left(\omega_{\mathrm{SI}}(\mathrm{t})+\pi \mathrm{J}_{\mathrm{SI}}\right)+\left(\omega_{\mathrm{I}}(\mathrm{t})+\omega_{\mathrm{I}}^{\text {iso }}\right)}{\omega_{1}}\right)^{2}+\ldots\right.} \\
& \left.-1-\frac{1}{2} \cdot\left(\frac{\left(\omega_{\mathrm{SI}}(\mathrm{t})+\pi \mathrm{J}_{\mathrm{SI}}\right)-\left(\omega_{\mathrm{I}}(\mathrm{t})+\omega_{\mathrm{I}}^{\text {iso }}\right)}{\omega_{1}}\right)^{2}-\ldots\right]
\end{aligned}
$$

Truncating Eq. [2.9] after the first two terms is equivalent to second-order perturbation theory and leads to the following approximate expression for the transition frequencies:

$$
\omega^{(3,4)}(t) \approx \omega_{S}^{\text {iso }}+\omega_{S}(t) \pm\left(\frac{\left(\omega_{S I}(t)+\pi J_{S I}\right) \cdot\left(\omega_{I}(t)+\omega_{I}^{\text {iso }}\right)}{\omega_{1}}\right)
$$

The two transition frequencies describe a splitting of the S-spin line due to a secondorder recoupling of the heteronuclear coupling term (either anisotropic dipolar coupling or isotropic J coupling) with an I-spin chemical-shift term (either anisotropic chemical-shielding tensor or isotropic chemical shift).

Assuming that the MAS rotation is slow compared to the time scale of the rf nutation $\left(\omega_{\mathrm{r}} \ll \omega_{1}\right)$, one can now apply average Hamiltonian theory to the transition frequencies to eliminate the time dependence due to the sample rotation:

$$
\begin{aligned}
\bar{\omega}^{(3,4)}= & \frac{1}{\tau_{\mathrm{r}}} \int_{0}^{\tau_{\mathrm{r}}} \omega^{(3,4)}(\mathrm{t}) \cdot \mathrm{d} \mathrm{t} \approx \frac{1}{\tau_{\mathrm{r}}} \int_{0}^{\tau_{\mathrm{r}}}\left(\omega_{\mathrm{S}}^{\text {iso }}+\omega_{\mathrm{S}}(\mathrm{t}) \pm\left(\frac{\left(\omega_{\mathrm{SI}}(\mathrm{t})+\pi \mathrm{J}_{\mathrm{SI}}\right) \cdot\left(\omega_{\mathrm{I}}(\mathrm{t})+\omega_{\mathrm{I}}^{\text {iso }}\right)}{\omega_{1}}\right)\right) d t \\
= & \omega_{\mathrm{S}}^{\text {iso }} \pm \frac{\pi \mathrm{J}_{\mathrm{SI}} \cdot \omega_{\mathrm{I}}^{\text {iso }}}{\omega_{1}} \\
& +\frac{1}{\tau_{\mathrm{r}}} \int_{0}^{\tau_{\mathrm{r}}} \omega_{\mathrm{S}}(\mathrm{t}) \mathrm{dt} \pm \frac{1}{\omega_{1} \tau_{\mathrm{r}}} \int_{0}^{\tau_{\mathrm{r}}}\left(\pi \mathrm{J}_{\mathrm{SI}} \omega_{\mathrm{I}}(\mathrm{t})+\omega_{\mathrm{I}}^{\text {iso }} \omega_{\mathrm{SI}}(\mathrm{t})+\omega_{\mathrm{SI}}(\mathrm{t}) \omega_{\mathrm{I}}(\mathrm{t})\right) \cdot \mathrm{dt}
\end{aligned}
$$

Here, the integration represents the time average over a full rotor period, $\tau_{\mathrm{r}}=2 \pi / \omega_{\mathrm{r}}$.

All purely second-rank tensor quantities in Eq. [2.11], i.e., the product of a scalar with a second-rank tensor, will be averaged out by the rotation about the magic angle. Therefore, we will not be able to observe a contribution due to the cross term 
between the scalar J coupling and the anisotropic chemical-shielding tensor or due to the cross term between the isotropic chemical shift and the anisotropic dipolarcoupling tensor.

The purely scalar cross term $\left(\pi \mathrm{J}_{\mathrm{SI}} \cdot \omega_{\mathrm{I}}^{\text {iso }}\right) / \omega_{1}$ represents the off-resonance decoupling term as is known from liquid-state NMR (3),(4). For a ${ }^{1} \mathrm{~J}_{\mathrm{CH}}$ coupling constant of about $130 \mathrm{~Hz}$ and an isotropic chemical-shift offset of $10 \mathrm{ppm}$, corresponding to $6 \mathrm{kHz}$ at $600 \mathrm{MHz}$ proton resonance frequency, we obtain a splitting of only $7.8 \mathrm{~Hz}$ assuming an rf-field strength of $100 \mathrm{kHz}$. Since the line width of rigid solids is only rarely below $10 \mathrm{~Hz}$, such a splitting can usually not be observed.

The last term in Eq. [2.11] represents the coupling of two second-rank tensors, namely the chemical-shielding tensor of the I spin and the heteronuclear dipolarcoupling tensor. The product of two second-rank tensors can generally be described by a weighted sum of a zeroth-rank, a second-rank, and a fourth-rank tensor (5). Therefore, this term will not be fully averaged out by a rotation about the magic angle and needs to be analyzed in more detail.

We will calculate the averaging by the sample rotation in a more general way as a rotation about an arbitrary axis inclined at an angle, $\theta_{r}$, with respect to the static magnetic field. To do this, we have to consider the transformations of both secondrank tensors from their respective principal-axes systems (PAS) into the laboratoryfixed coordinate system (Figure 2.1). The chemical-shielding tensor is first rotated into the PAS system of the dipolar-coupling tensor. Then both the chemical-shielding tensor and the dipolar-coupling tensor are rotated into the rotor-fixed frame from which they are subsequently rotated into the laboratory-fixed coordinate system. This leads to the following time-dependent transformation for the two tensors:

$$
\begin{gathered}
\omega_{\mathrm{SI}}(\mathrm{t}, \Omega)=\frac{1}{\sqrt{6}} \sum_{\mathrm{n}=2}^{-2} \mathfrak{D}_{\mathrm{n}, 0}^{2}\left(-\omega_{\mathrm{r}} \mathrm{t},-\theta_{\mathrm{r}}, 0\right) \cdot \mathfrak{D}_{0, \mathrm{n}}^{2}(\alpha, \beta, \gamma) \cdot \rho_{2,0}^{\mathrm{SI}} \\
\omega_{\mathrm{I}}(\mathrm{t}, \Omega)=\frac{2}{\sqrt{6}} \sum_{\mathrm{m}=-2}^{2} \sum_{\mathrm{m}^{\prime}=-2}^{2} \sum_{\mathrm{m}^{\prime \prime}=-2}^{2} \mathfrak{D}_{\mathrm{m}, 0}^{2}\left(-\omega_{\mathrm{r}} \mathrm{t},-\theta_{\mathrm{r}}, 0\right) \cdot \mathfrak{D}_{\mathrm{m}^{\prime}, \mathrm{m}}^{2}(\alpha, \beta, \gamma) \\
\times \mathscr{D}_{\mathrm{m}^{\prime \prime}, \mathrm{m}^{\prime}}^{2}\left(\alpha_{\mathrm{I}}, \beta_{\mathrm{I}}, \gamma_{\mathrm{I}}\right) \cdot \rho_{2, \mathrm{~m}^{\prime \prime}}^{\mathrm{I}}
\end{gathered}
$$


The Wigner rotation-matrix elements $\mathfrak{D}_{\mathrm{m}, \mathrm{n}}^{2}(\alpha, \beta, \gamma)$ are defined according to the conventions presented in Ref. (5). The sample spinning frequency is $\omega_{\mathrm{r}} ; \Omega=(\alpha, \beta, \gamma)$ is the set of Euler angles which describes the transformation from the principal-axes system of the dipolar-coupling tensor to the rotor-fixed coordinate system (powder average); and $\left(\alpha_{\mathrm{I}}, \beta_{\mathrm{I}}, \gamma_{\mathrm{I}}\right)$ are the three Euler angles describing the orientation of the Ispin chemical-shielding tensor in the principal-axes system of the dipolar-coupling tensor. The spherical-tensor notation of the two tensors $\left(\rho_{2, m}\right.$ elements) is defined in Appendix A.

We can now insert Eqs. [2.12] and [2.13] into Eq. [2.11] and simplify the resulting expression. The time-averaged product of two second-rank tensors has, to zeroth-order approximation, non-vanishing contributions only under the condition $\mathrm{m}=-\mathrm{n}$ where $\mathrm{m}$ and $\mathrm{n}$ are the summation indices in Eqs. [2.12] and [2.13], respectively. Under this condition Eq. [2.11] can be substantially simplified, and one obtains the following result for the time-averaged transition frequencies:

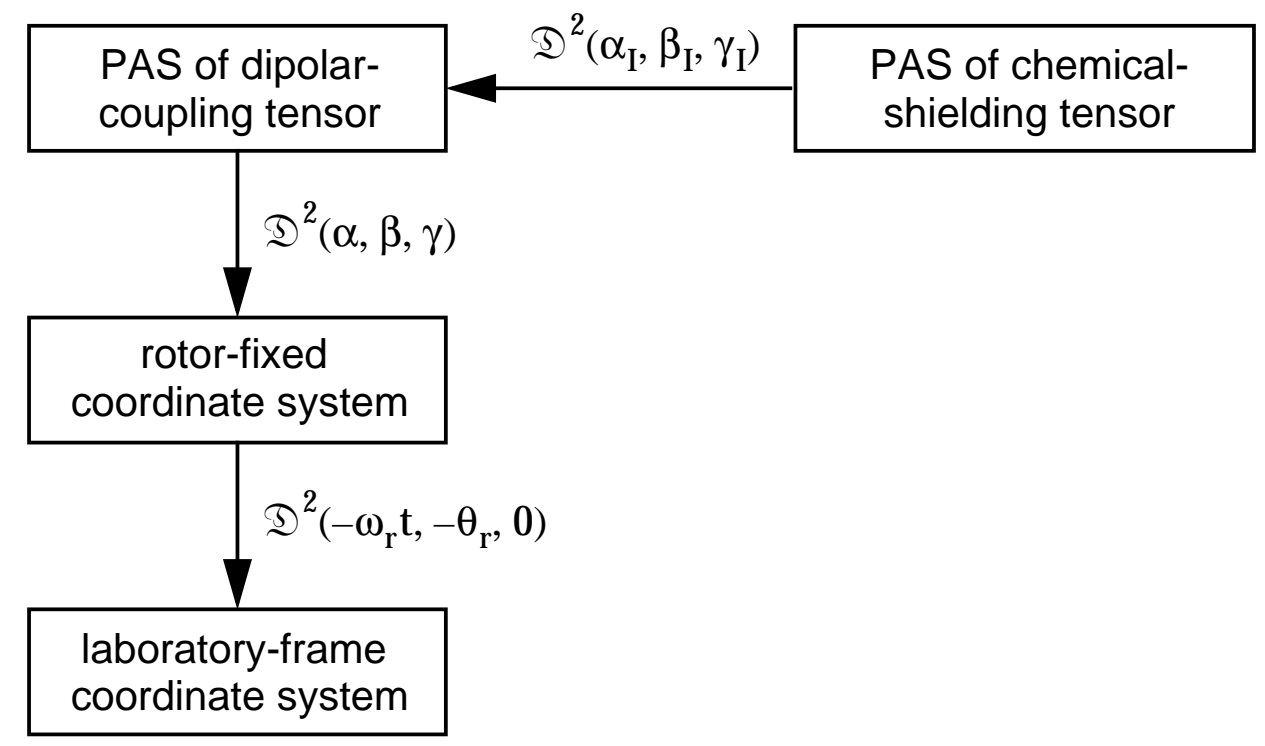

Figure 2.1: Sequence of transformations and Euler angles necessary to rotate the two tensors from their respective principal-axes systems into the laboratory-fixed coordinate system. The chemical-shielding tensor is first rotated into the principal-axes system of the dipolar coupling tensor $\left(\mathfrak{D}^{2}\left(\alpha_{\mathrm{I}}, \beta_{\mathrm{I}}, \gamma_{\mathrm{I}}\right)\right)$, then both tensors are rotated into the rotor-fixed frame $\left(\mathfrak{D}^{2}(\alpha, \beta, \gamma)\right)$, and from there, finally, into the laboratory-fixed frame $\left(\mathfrak{D}^{2}\left(-\omega_{\mathrm{r}} \mathrm{t},-\theta_{\mathrm{r}}, 0\right)\right)$. 


$$
\begin{aligned}
\bar{\omega}^{(3,4)}(\Omega)= & \omega_{\mathrm{S}}^{\text {iso }} \pm \frac{\pi \mathrm{J}_{\mathrm{SI}} \cdot \omega_{\mathrm{I}}^{\text {iso }}}{\omega_{1}} \\
& +\sqrt{\frac{2}{3}} \mathrm{~d}_{0,0}^{2}\left(\theta_{\mathrm{r}}\right) \sum_{\mathrm{m}^{\prime}=-2}^{2} \mathfrak{D}_{\mathrm{m}^{\prime}, 0}^{2}(\alpha, \beta, 0) \sum_{\mathrm{m}^{\prime \prime}=-2}^{2} \mathfrak{S}_{\mathrm{m}^{\prime \prime}, \mathrm{m}^{\prime}}^{2}\left(\alpha_{\mathrm{S}}, \beta_{\mathrm{S}}, 0\right) \cdot \rho_{2, \mathrm{~m}^{\prime \prime}}^{\mathrm{S}} \\
& \pm \frac{\pi \mathrm{J}_{\mathrm{SI}}}{\omega_{1}} \cdot \sqrt{\frac{2}{3}} \mathrm{~d}_{0,0}^{2}\left(\theta_{\mathrm{r}}\right) \sum_{\mathrm{m}^{\prime}=-2}^{2} \mathfrak{D}_{\mathrm{m}^{\prime}, 0}^{2}(\alpha, \beta, 0) \sum_{\mathrm{m}^{\prime \prime}=-2}^{2} \mathfrak{D}_{\mathrm{m}^{\prime \prime}, \mathrm{m}^{\prime}}^{2}\left(\alpha_{\mathrm{I}}, \beta_{\mathrm{I}}, 0\right) \cdot \rho_{2, \mathrm{~m}^{\prime \prime}}^{\mathrm{I}} \\
& \pm \frac{\omega_{\mathrm{I}}^{\mathrm{iso}}}{\omega_{1}} \cdot \frac{1}{\sqrt{6}} \mathrm{~d}_{0,0}^{2}\left(\theta_{\mathrm{r}}\right) \sum_{\mathrm{n}=2}^{-2} \mathfrak{D}_{0, \mathrm{n}}^{2}(\alpha, \beta, 0) \cdot \rho_{2,0}^{\mathrm{SI}} \\
& \pm \frac{1}{3 \omega_{1}} \cdot \rho_{2,0}^{\mathrm{SI}} \cdot \sum_{\mathrm{j}=0,2,4} \mathrm{C}(2,2, \mathrm{j} ; 0,0) \cdot \mathrm{d}_{0,0}^{\mathrm{j}}\left(\theta_{\mathrm{r}}\right) \\
& \times \sum_{\mathrm{m}^{\prime}=-2}^{2} \mathfrak{D}_{\mathrm{m}^{\prime}, 0}^{\mathrm{j}}(\alpha, \beta, 0) \cdot \mathrm{C}\left(2,2, \mathrm{j} ; 0, \mathrm{~m}^{\prime}\right) \\
& \times \sum_{\mathrm{m}^{\prime \prime}=-2}^{2} \mathfrak{D}_{\mathrm{m}^{\prime \prime}, \mathrm{m}^{\prime}}^{2}\left(\alpha_{\mathrm{I}}, \beta_{\mathrm{I}}, 0\right) \cdot \rho_{2, \mathrm{~m}^{\prime \prime}}^{\mathrm{I}}
\end{aligned}
$$

Here, $C\left(j_{1}, j_{2}, j ; m_{1}, m_{2}\right)$ are the Clebsch-Gordan coefficients as defined in Ref. (6) and $\mathrm{d}_{\mathrm{m}, \mathrm{n}}^{\mathrm{j}}(\beta)$ are reduced Wigner rotation-matrix elements (5). The angle $\gamma$ was assumed to be zero since it only changes the orientation of the rotor at the start of the experiment. All other symbols have been defined previously.

The first term in Eq. [2.14] represents the isotropic chemical shift of spin $S$, the second term the isotropic splitting due to off-resonance decoupling (3),(4). The third term describes the chemical-shielding tensor of the $S$ spin, the fourth term is the cross term between the isotropic J coupling and the chemical-shielding tensor of the I spin, and the fifth term is the cross term of the isotropic chemical shift of the I spin and the heteronuclear dipolar-coupling tensor. These latter three terms scale as second-rank tensors with $\mathrm{P}_{2}\left(\cos \theta_{\mathrm{r}}\right)=\mathrm{d}_{0,0}^{2}\left(\theta_{\mathrm{r}}\right)$. The last term in Eq. [2.14] is the cross term between the chemical-shielding tensor of the I spin and the heteronuclear dipolar-coupling tensor. It consists of the sum of three different terms $(j=0,2,4)$ which scale as a zerothrank, a second-rank, and a fourth-rank tensor under single-axis rotation.

Setting the angle of the rotation axis to $\theta_{\mathrm{r}}=0^{\circ}$ gives the solution for the static spectrum. Under this condition all scaling factors $\mathrm{d}_{0,0}^{\mathrm{j}}(0)=1$ and all terms contribute 
to the line shape of the S-spin resonance. If the inclination angle of the rotation axis with respect to the static magnetic field corresponds to the magic angle $\left(\theta_{\mathrm{r}}=\right.$ $\theta_{\mathrm{m}} \approx 54.74^{\circ}$ ), all second-rank tensor contributions to the transition frequencies will vanish, because $\mathrm{d}_{0,0}^{2}\left(\theta_{\mathrm{m}}\right)=0$. The chemical-shielding tensor of the $S$ spin, the cross term between the isotropic J coupling and the chemical-shielding tensor of the I spin, the cross term between the isotropic chemical shift of the I spin and the heteronuclear dipolar-coupling tensor, and the second-rank tensor contribution to the cross term between the chemical-shielding tensor and the heteronuclear dipolar-coupling tensor will be scaled to zero. Only the isotropic chemical shift of the S spin, the off-resonance decoupling term, and the isotropic and scaled fourth-rank tensor parts of the secondorder cross term remain:

$$
\begin{aligned}
\bar{\omega}^{(3,4)}(\Omega)= & \omega_{\mathrm{S}}^{\text {iso }} \pm \frac{\pi \mathrm{J}_{\mathrm{SI}} \cdot \omega_{\mathrm{I}}^{\text {iso }}}{\omega_{1}} \\
& \pm \frac{1}{3 \omega_{1}} \cdot \rho_{2,0}^{\mathrm{SI}} \cdot \sum_{\mathrm{j}=0,4} \mathrm{C}(2,2, \mathrm{j} ; 0,0) \cdot \mathrm{d}_{0,0}^{\mathrm{j}}\left(\theta_{\mathrm{m}}\right) \\
& \times \sum_{\mathrm{m}^{\prime}=-\mathrm{j}}^{\mathrm{j}} \mathfrak{D}_{\mathrm{m}^{\prime}, 0}^{\mathrm{j}}(\alpha, \beta, 0) \cdot \mathrm{C}\left(2,2, \mathrm{j} ; 0, \mathrm{~m}^{\prime}\right) \\
& \times \sum_{\mathrm{m}^{\prime \prime}=-2}^{2} \mathfrak{D}_{\mathrm{m}}^{2}, \mathrm{~m}^{\mathrm{m}}\left(\alpha_{\mathrm{I}}, \beta_{\mathrm{I}}, 0\right) \cdot \rho_{2, \mathrm{~m}^{\prime \prime}}^{\mathrm{I}}
\end{aligned}
$$

For each crystallite orientation, the spectrum consists of a doublet centered about the isotropic chemical-shift of the S spin. The splitting consists of an orientationindependent part given by the off-resonance decoupling term and by the zeroth-rank tensor part of the second-order cross term and an orientation-dependent part given by the fourth-rank tensor contribution to the second-order cross term.

It is, in principle, possible to average out both the second-rank and the fourthrank tensor components of Eq. [2.14] by using dynamic-angle spinning (DAS) (sequential averaging) or double rotation (DOR) (simultaneous averaging) techniques (7), (8), (9). In this case the resonance frequencies are given by 


$$
\bar{\omega}^{(3,4)}(\Omega)=\omega_{\mathrm{S}}^{\text {iso }} \pm\left(\frac{\pi \mathrm{J}_{\mathrm{SI}} \cdot \omega_{\mathrm{I}}^{\text {iso }}}{\omega_{1}}+\frac{1}{15 \omega_{1}} \cdot \rho_{2,0}^{\mathrm{SI}} \cdot \sum_{\mathrm{m} "=-2}^{2} \mathfrak{D}_{\mathrm{m} ", 0}^{2}\left(\alpha_{\mathrm{I}}, \beta_{\mathrm{I}}, 0\right) \cdot \rho_{2, \mathrm{~m}}^{\mathrm{I}}\right)
$$

and the resulting second-order splitting is fully isotropic and gives rise to a spectrum that consists of a sharp doublet centered about the isotropic chemical shift of the $S$ spin. The splitting of the line is determined by the off-resonance decoupling term and the isotropic part $\left(\mathrm{m}^{\prime \prime}=0\right)$ of the second-order cross term.

Equations [2.14] and [2.15] allow very efficient simulation of second-order spectra, which is important if extraction of parameters from experimental spectra is desired. Non-linear least-square fitting to obtain parameters (e.g., orientation of the two tensors) and their corresponding error ranges is, in principle, possible based on this analytical solution assuming that the two-spin model is a good approximation.

\subsubsection{Simultaneous Averaging of Space and Spin Parts}

We can also calculate the average Hamiltonian for an SI two-spin system by simultaneously time averaging over the MAS rotation and over the rotation due to interaction-frame transformation by the rf field. Here, we have to assume that $\omega_{1} / \omega_{\mathrm{r}}=\mathrm{p}$ is an integer, i.e., we assume that the two rotations are synchronized. If we avoid the rotary-resonance conditions, i.e., by assuming $p>2$, we obtain for the zeroth-order average Hamiltonian:

$$
\overline{\mathscr{H}}^{(0)}=\mathscr{H}_{\mathrm{S}}^{\mathrm{CS}}=\omega_{\mathrm{S}}^{\text {iso }} \cdot \mathrm{S}_{\mathrm{z}}
$$

and for the first-order average Hamiltonian:

$$
\begin{aligned}
\overline{\mathscr{H}}^{(1)}= & \frac{1}{\omega_{\mathrm{r}}}\left[\frac{\pi \mathrm{J}_{\mathrm{SI}} \omega_{\mathrm{I}}^{\text {iso }}}{\mathrm{p}}+\frac{-4 \mathrm{p}+\mathrm{p}^{3}}{4-5 \mathrm{p}^{2}+\mathrm{p}^{4}}\left(\omega_{\mathrm{SI}}^{+1} \omega_{\mathrm{I}}^{-1}+\omega_{\mathrm{SI}}^{-1} \omega_{\mathrm{I}}^{+1}\right)\right. \\
& \left.+\frac{-\mathrm{p}+\mathrm{p}^{3}}{4-5 \mathrm{p}^{2}+\mathrm{p}^{4}}\left(\omega_{\mathrm{SI}}^{+2} \omega_{\mathrm{I}}^{-2}+\omega_{\mathrm{SI}}^{-2} \omega_{\mathrm{I}}^{+2}\right)\right] \cdot 2 S_{\mathrm{z}} \mathrm{I}_{\mathrm{x}}
\end{aligned}
$$

For simplicity only terms involving S-spin operators are shown. The constants $\omega_{\mathrm{SI}}^{\mathrm{m}}$ and $\omega_{\mathrm{I}}^{\mathrm{m}}$ are the Fourier coefficients of the time-dependent heteronuclear dipolar 
coupling and the I-spin chemical-shielding tensor, respectively. They are defined in Appendix A. For large values of p, we can simplify Eq. [2.18] and obtain:

$$
\overline{\mathscr{H}}^{(1)}=\left[\frac{\pi \mathrm{J}_{\mathrm{SI}} \omega_{\mathrm{I}}^{\text {iso }}}{\mathrm{p} \omega_{\mathrm{r}}}+\sum_{\substack{\mathrm{m}=-2 \\ \mathrm{~m} \neq 0}}^{2} \frac{\omega_{\mathrm{SI}}^{-\mathrm{m}} \omega_{\mathrm{I}}^{+\mathrm{m}}}{\mathrm{p} \omega_{\mathrm{r}}}\right] 2 \mathrm{~S}_{\mathrm{z}} \mathrm{I}_{\mathrm{x}}=\left[\frac{\pi \mathrm{J}_{\mathrm{SI}} \omega_{\mathrm{I}}^{\text {iso }}}{\omega_{1}}+\sum_{\substack{\mathrm{m}=-2 \\ \mathrm{~m} \neq 0}}^{2} \frac{\omega_{\mathrm{SI}}^{-\mathrm{m}} \omega_{\mathrm{I}}^{+\mathrm{m}}}{\omega_{1}}\right] 2 \mathrm{~S}_{\mathrm{z}} \mathrm{I}_{\mathrm{x}} .
$$

The result obtained in Eq. [2.19] is fully equivalent to the result obtained from sequential averaging in Eq. [2.11]. This can easily be seen by expanding the timedependent chemical-shielding tensor and the dipolar-coupling tensor of Eq. [2.11] as Fourier series (see Eqs. [7.6] and [7.8] in Appendix A) and keeping only the timeindependent terms. Both approaches clearly show that the cross term between the chemical-shielding tensor and the heteronuclear dipolar-coupling tensor dominates the residual line width in high-power cw decoupled spectra of isolated spin pairs.

\subsection{Numerical Simulations}

Simulations to analyze the behavior of an isolated two-spin system were carried out using the NMR simulation environment GAMMA (10). Numerical values close to the values found for the model compound, [ $\left.\mathrm{d}_{9}\right]$-trimethyl- ${ }^{15} \mathrm{~N}$-ammonium chloride (see Fig. 1.5) were used in the numerical simulations. The anisotropy of the dipolar-coupling tensor was set to $\delta_{\mathrm{SI}} /(2 \pi)=20 \mathrm{kHz}$, and the chemical-shielding tensor was assumed to be axially symmetric $\left(\eta_{\mathrm{I}}=0\right)$ with an anisotropy of $\delta_{\mathrm{I}} /(2 \pi)=$ $6.8 \mathrm{kHz}$. The two tensors were assumed to be coaxial unless otherwise mentioned.

The accuracy of simulations based on the approximate analytical solution from second-order perturbation theory (Equation [2.15]) was tested by comparing exact numerical simulations calculated by small-step integration of the time-dependent Hamiltonian with simulations based on the analytical solution at different rf-field strengths. The numerical simulations were done for an MAS frequency of $\omega_{\mathrm{r}} /(2 \pi)=$ $5 \mathrm{kHz}$ and with 5000 time steps per rotor cycle leading to a $40 \mathrm{~ns}$ time resolution. Simulations for 300 different crystallite orientations was summed and the method of Cheng et al. (11) was used to obtain optimum coverage of the sphere. The dwell time 
was set to one third of the rotor cycle (full SW $=15 \mathrm{kHz}$ ), and 4096 data points were computed. The spectra were processed using an exponential line broadening of $100 \mathrm{~Hz}$. The spectra based on the approximate analytical solution of the timeaveraged Hamiltonian were calculated directly in the frequency domain. All parameters were the same as in the time-domain simulations except that 10000 different powder orientations were summed. The resulting frequency-domain spectra were convolved with a 100-Hz Lorentzian line. The spectra in Figure 2.2 are calculated for three different rf-field strengths $\omega_{1} /(2 \pi)=70 \mathrm{kHz}, 30 \mathrm{kHz}$, and

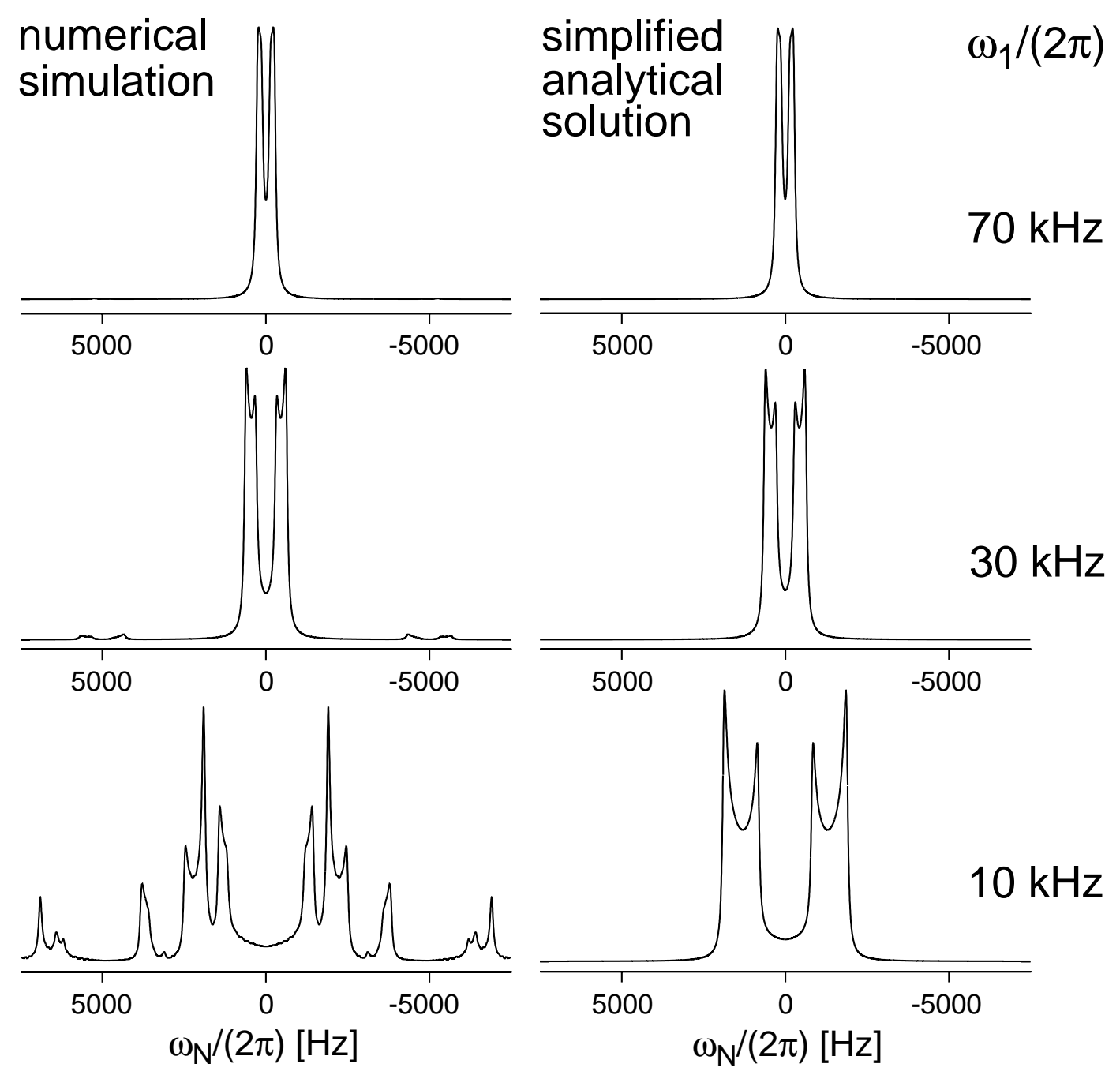

Figure 2.2: Comparison of simulations based on small-step numerical integration of the timedependent Hamiltonian and simulations based on the approximate analytical solution (Equation [2.15]) for three different decoupling field strengths. The two methods of simulations at $\omega_{1} /(2 \pi)=70$ and $30 \mathrm{kHz}$ show differences on the order of one percent. The simulations at $\omega_{1} /(2 \pi)=10 \mathrm{kHz}$ are very different from each other, which reflects the fact that the assumptions made in the second-order truncation (Equation [2.8]) are not valid here. 
$10 \mathrm{kHz}$. For the two higher rf-field strengths the two simulation methods agree very well with differences in the central line on the order of one percent. These small deviations are most likely due to the truncation terms higher than second order in the analytical solution or to the relatively low number of powder points used in the timedomain simulation. The spinning sidebands at $\pm 5000 \mathrm{~Hz}$ observed in the numerical simulations are not present in the analytical solution because it is based on the assumption of a stroboscopic observation. For $\omega_{1} /(2 \pi)=10 \mathrm{kHz}$ (Fig. 2.2c), the differences are large and the line shapes of the two spectra do not agree at all. This is due to the breakdown of the assumptions made in deriving the analytical solution in Equation [2.15]. At an rf-field strength of $10 \mathrm{kHz}$ the decoupling field strength is no longer greater than the magnitudes of the chemical-shielding and the dipolarcoupling tensors. In addition, the $n=2$ rotary-resonance condition (12),(13),(14),(15) is fulfilled, which cannot be described by a sequential averaging approach as was used in deriving the approximate analytical equation. The advantage of the frequencydomain simulation based on the analytical solution is its computation speed. The frequency-domain simulation is about 6000 times faster than the time-domain simulation using small step integration of the Liouville-von-Neumann equation.

Figure 2.3 shows simulations of the second-order recoupled spectra under static (a), magic-angle sample spinning (b), and double-rotation (c) conditions illustrating the averaging properties of the second-order recoupled Hamiltonian under spatial rotation. The simulations were performed as frequency-domain simulations based on the analytical solution shown in Equation [2.14]. The decoupling field strength was $\omega_{1} /(2 \pi)=71.5 \mathrm{kHz}$. Ten-thousand different crystallite orientations were summed, and the resulting spectrum was convolved with a Lorentzian line of width $50 \mathrm{~Hz}$. The static spectrum (Figure 2.3a) is very broad with a full width at the base of $1838 \mathrm{~Hz}$. It is the result of a superposition of the isotropic splitting with both the second-rank and the fourth-rank contributions to the secondorder Hamiltonian. The MAS spectrum (Figure 2.3b) shows a pure fourth-rank tensor powder pattern superimposed on the isotropic splitting with a full width of $528 \mathrm{~Hz}$ close to the bottom of the line. The DOR spectrum (Figure 2.3c) shows, as expected, two sharp lines with an isotropic splitting of $363 \mathrm{~Hz}$. 
The dependence of the second-order recoupled spectra on the relative orientation of the two tensors is illustrated in Figure 2.4. The simulations were done as frequency-domain simulations using the same parameters as for the spectra shown in Fig. 2.3. It can clearly be seen that there is a strong dependence of the line shape on the angle $\beta_{\text {I }}$ as expected from Eq. [2.15]. It is not straightforward to predict the line shape from Eq. [2.15], especially if the chemical-shielding tensor is not axially symmetric. The simulations of Fig. 2.4, however, show that the variations could be strong enough to allow the determination of the angle between the two tensors from this type of second-order spectrum, assuming that the investigated system can indeed be described by an isolated two-spin system.

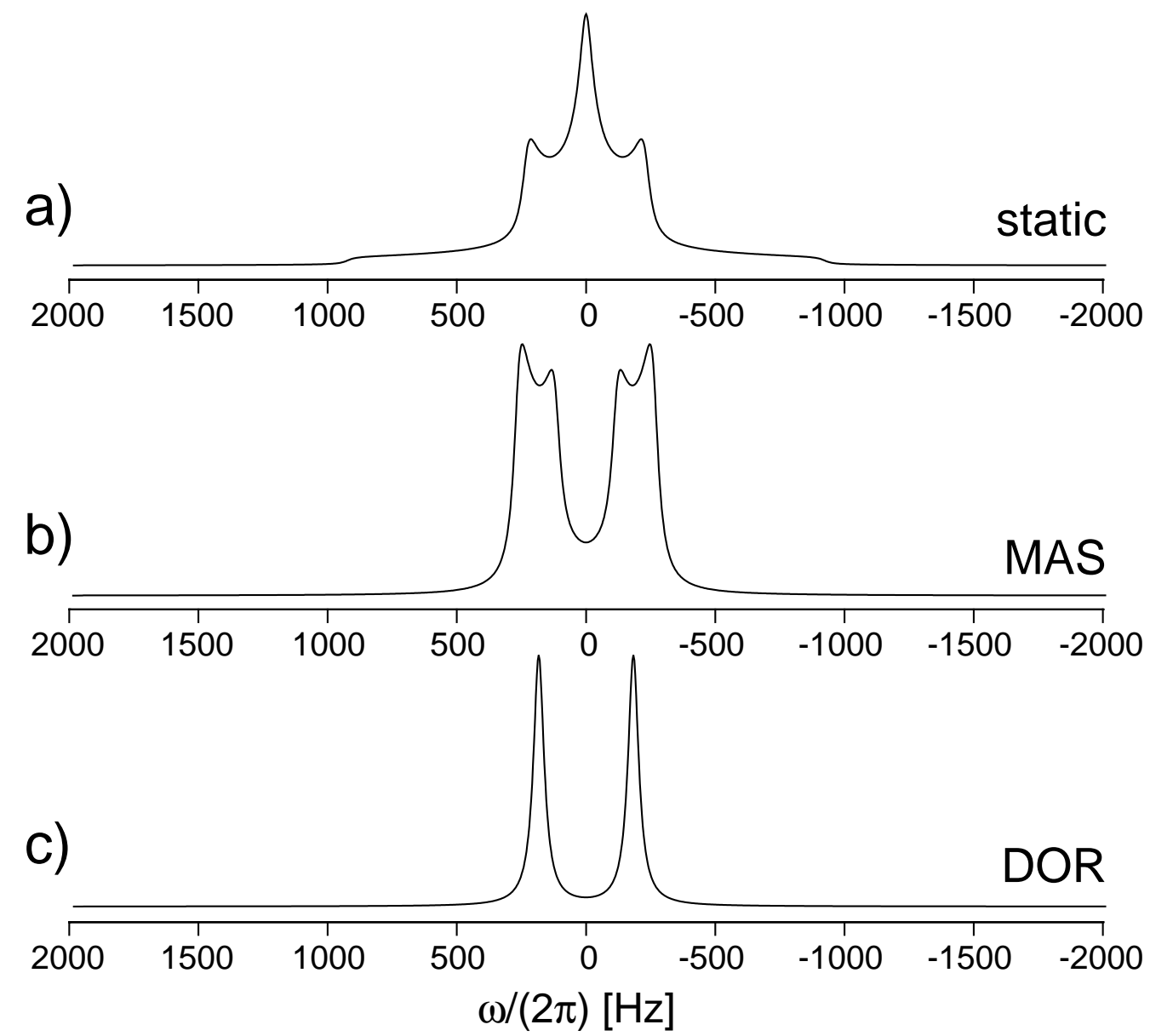

Figure 2.3: Simulated second-order line shape under static, magic-angle spinning, and double-rotation conditions. The static spectrum is very broad and shows a superposition of a zeroth-rank, a second-rank, and a fourth-rank tensor contribution to the Hamiltonian. The MAS spectrum is a pure fourth-rank tensor powder pattern superimposed on an isotropic splitting. The DOR spectrum shows only the isotropic splitting. 


\subsection{Experimental Results}

As a model substance for an isolated two-spin system, fully ${ }^{15} \mathrm{~N}$-labeled tri(trideuteromethyl)-ammonium chloride $\left({ }^{1} \mathrm{H}_{-}{ }^{15} \mathrm{~N}\right.$ spin system) was used. The synthesis of $\left[\mathrm{d}_{10}\right]$-trimethyl $-{ }^{15} \mathrm{~N}$-ammonium chloride is described in the literature (16). The shortest nitrogen-nitrogen distance calculated from the crystallographic data for trimethyl-ammonium chloride (17) is $5.7 \AA$ corresponding to a dipolar-coupling constant of $\delta_{\mathrm{NN}} /(2 \pi)=13.3 \mathrm{~Hz}$. The shortest proton-proton distance is of similar magnitude in the methyl-deuterated compound. The chemical-shielding tensor of ${ }^{15} \mathrm{~N}$ in this compound is very small. A static proton-decoupled spectrum (data not shown) using a symmetric $(2 \pi)_{+x},(2 \pi)_{-x}$ sequence resulted in a Gaussian line with a half width at half height of $290 \mathrm{~Hz}$. The chemical-shielding tensor of the proton was not measured directly. Its anisotropy was obtained from fitting the second-order MAS spectrum assuming that the PAS of the chemical-shielding tensor of the proton and the PAS of the dipolar-coupling tensor are coaxial. The value obtained from these fits was $\delta_{\mathrm{H}} /(2 \pi)=6800 \pm 200 \mathrm{~Hz}$. This value agrees with independent measurements of the chemical-shielding tensor (18). The anisotropy of the dipolar coupling of the ${ }^{15} \mathrm{~N}$ -
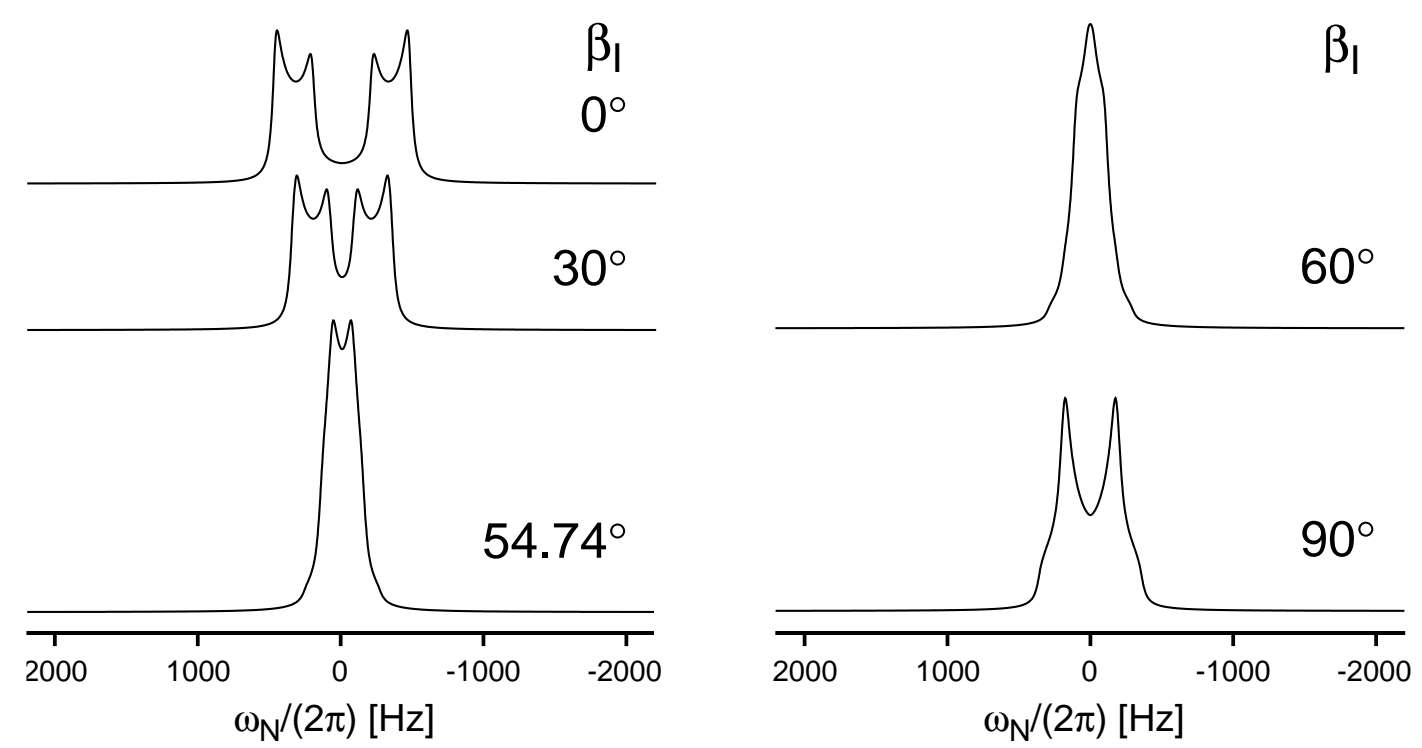

Figure 2.4: Dependence of the second-order recoupled spectrum on the orientation of the two tensors. The chemical shielding tensor was assumed to be axially symmetric so only one parameter $\left(\beta_{\mathrm{I}}\right)$ is needed to describe the orientation of the principal-axes systems of the two tensors. The line shape of the spectra depends very strongly on the relative orientation. 
${ }^{1} \mathrm{H}$ spin pair was measured from static and slow-spinning MAS spectra and was found to be $\delta_{\mathrm{NH}} /(2 \pi)=20110 \pm 210 \mathrm{~Hz}$ corresponding to a distance of $\mathrm{r}_{\mathrm{NH}}=$ $1.066 \pm 0.004 \AA$.

Figure 2.5 shows one-dimensional ${ }^{15} \mathrm{~N}$ spectra acquired without (a) and with (b and c) decoupling of the protons by cw irradiation. All three spectra were recorded at an MAS frequency of $30 \mathrm{kHz}$. The spectrum without decoupling (Figure 2.5a) shows a sharp doublet with a splitting equal to the known one-bond $\mathrm{J}_{\mathrm{NH}^{-}}$-coupling of about $100 \mathrm{~Hz}$. The appearance of the doublet indicates that the spin-diffusion rate

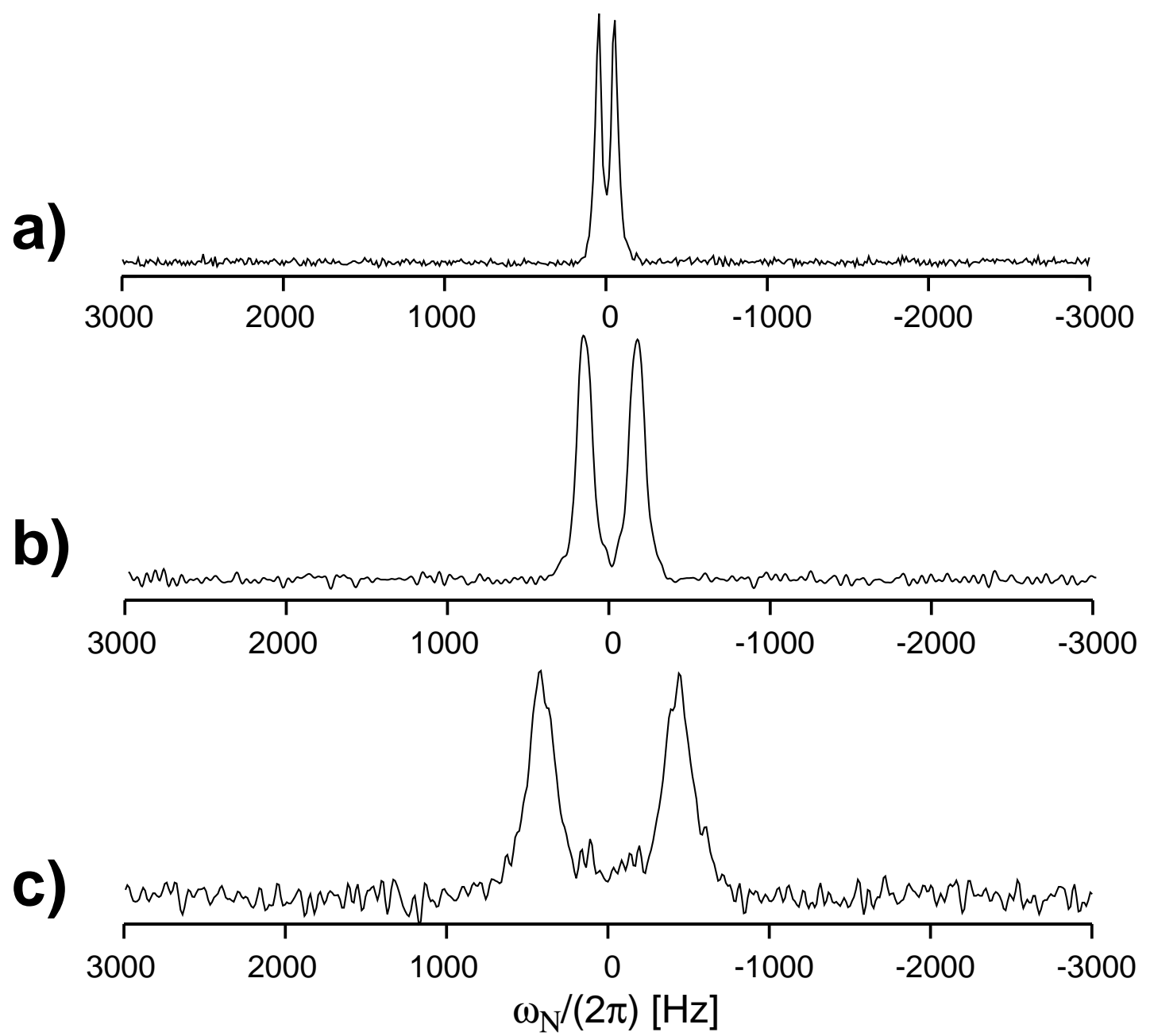

Figure 2.5: (a) ${ }^{15} \mathrm{~N}$ spectrum of $\left[\mathrm{d}_{9}\right]$-trimethyl- ${ }^{15} \mathrm{~N}$-ammonium chloride without proton decoupling recorded at an MAS spinning frequency of $30 \mathrm{kHz}$. The visible splitting is the one-

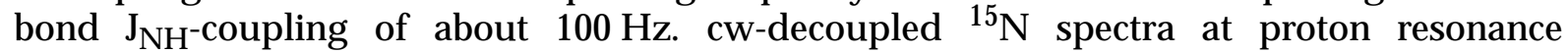
frequencies of (b) $300 \mathrm{MHz}$ and (c) $600 \mathrm{MHz}$ at an MAS spinning frequency of $30 \mathrm{kHz}$ and a decoupling field strength of $100 \mathrm{kHz}$. 
constant is below $100 \mathrm{~Hz}$ (19). Figures $2.5 \mathrm{~b}$ and $2.5 \mathrm{c}$ show the $\mathrm{cw}$-decoupled ${ }^{15} \mathrm{~N}$ spectra $\left(\omega_{1} /(2 \pi)=100 \mathrm{kHz}\right)$ of $\left[\mathrm{d}_{9}\right]$-trimethyl $-{ }^{15} \mathrm{~N}$-ammonium chloride at an MAS frequency of $30 \mathrm{kHz}$ and at proton resonance frequencies of $600 \mathrm{MHz}$ (Figure 2.5b) and $300 \mathrm{MHz}$ (Figure 2.5c). Due to the high spinning frequency and the perdeuteration of the methyl groups, the proton spin-diffusion rate constant is quite small, and the cross-term between the dipolar-coupling tensor and the CSA tensor dominates the spectrum. Therefore, we observe two well-separated lines with a splitting proportional to the proton resonance frequency. The fine structure visible in the numerical simulations (Figure 2.4) is not observed in the experimental ${ }^{15} \mathrm{~N}$ spectrum of trimethyl-ammonium chloride.

Figure 2.6 shows a series of ${ }^{15} \mathrm{~N}$ spectra where the proton decoupling field strengths was varied from $\omega_{1} /(2 \pi)=17$ to $72 \mathrm{kHz}$. The spectra were recorded at an MAS spinning frequency of $\omega_{\mathrm{r}} /(2 \pi)=5 \mathrm{kHz}$. The line widths near the base of the

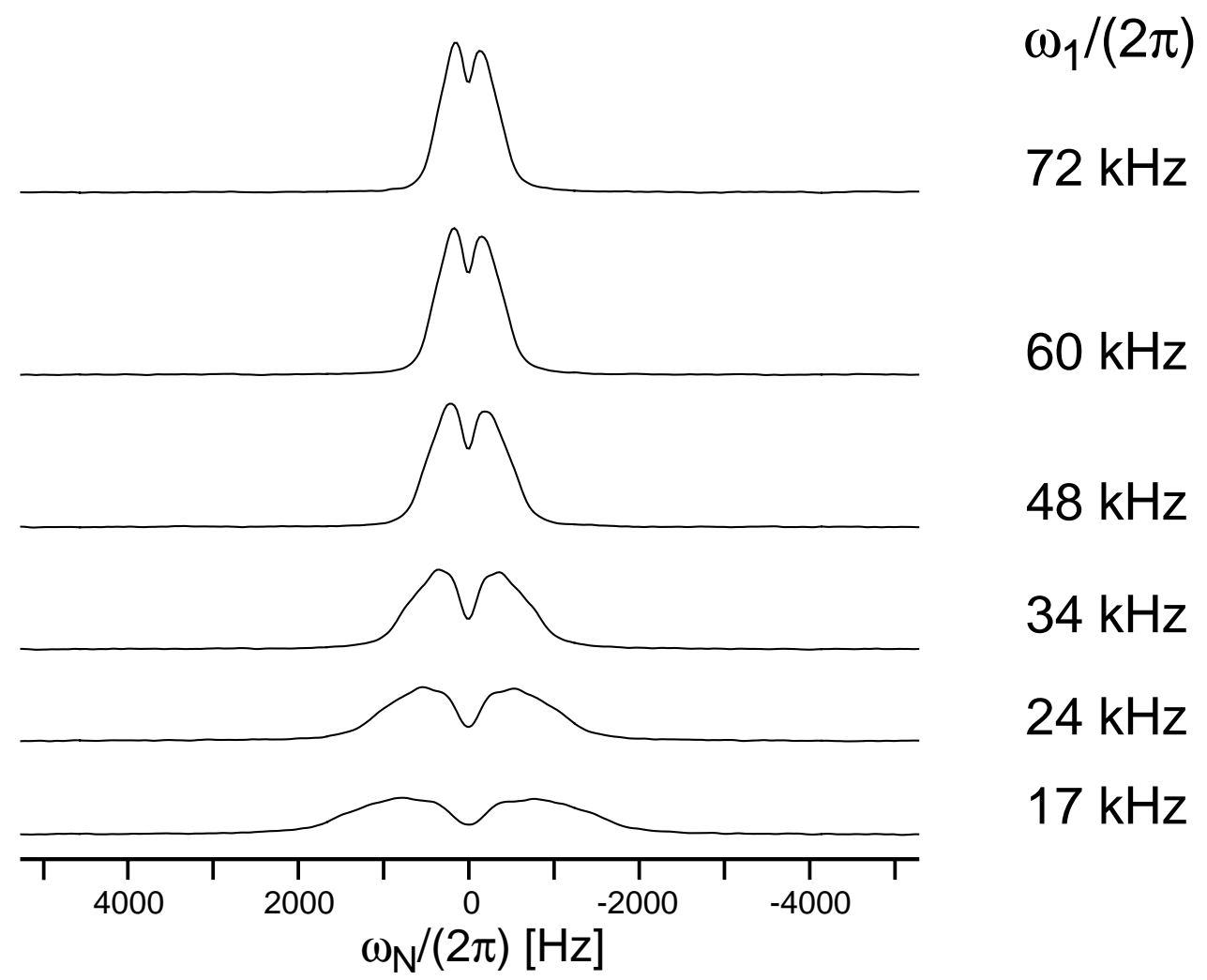

Figure 2.6: ${ }^{15} \mathrm{~N}$ spectra of ${ }^{15} \mathrm{~N}$-labeled tri-(trideuteromethyl)-ammonium chloride as a function of the proton decoupling power. The decoupling field strength was varied from $\omega_{1} /(2 \pi)=17$ to $72 \mathrm{kHz}$. The linewidth scales to a good approximation with $1 / \omega_{1}$. 
peaks at different decoupling powers correlate very well with the inverse of the proton decoupling field strength as expected from the theoretical calculations shown in Chapter 2.1. This suggests that neglecting all terms higher than second order in the perturbation expansion of Equation [2.11] was justified.

As mentioned already in Chapter 2.1, the second-order recoupling can be made isotropic by performing the experiment under DOR or DAS conditions. The pulse sequence used to record an isotropic second-order recoupled spectrum under DAS is shown in Figure 2.7. The experiment was implemented as a pure-phase experiment (20) in $t_{1}$ and uses States-type processing (21) to distinguish between positive and negative frequencies. Since the second-order recoupled Hamiltonian is quantized along the decoupling field of the I spins (Eq. [2.19]), additional storage pulses (22) are needed for the I spins before and after the change of the rotor axis in contrast to a standard pure-phase DAS experiment (20).

For the 2D-DAS experiment $50 \mathrm{t}_{1}$ times were recorded with 256 scans of 256 points in $t_{2}$ for each of the two complex data sets. During $t_{2}$, phase-alternating $2 \pi-$ pulse decoupling was employed to obtain a narrow line in $\omega_{2}$. The flipping time to

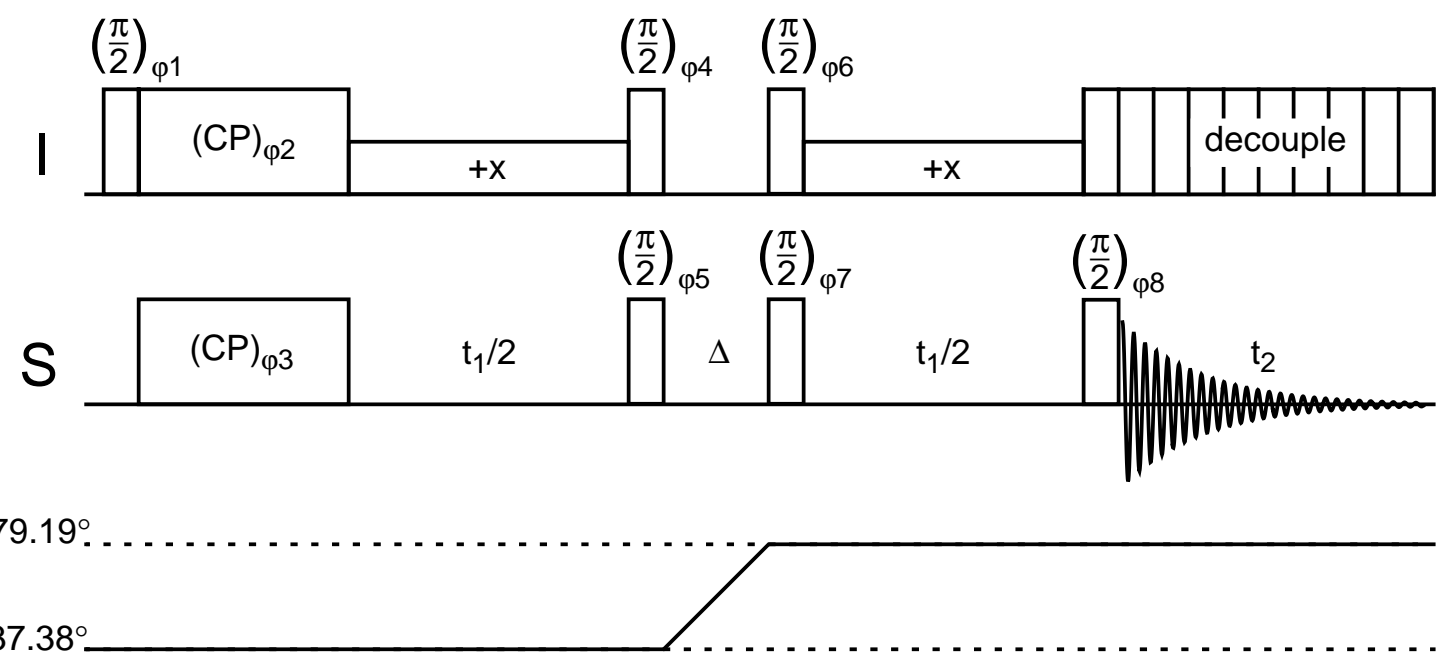

Figure 2.7: Pulse sequence used to measure the DAS spectrum of the second-order recoupling. The pulse sequence implements pure phase during $t_{1}$ by storing the appropriate orthogonal components during the change of the rotor axis. The two data sets, modulated as $\cos ^{2}\left(\omega t_{1} / 2\right)$ and $\sin ^{2}\left(\omega t_{1} / 2\right)$ are then summed. The rf-field strength during the two decoupling periods must be equal. 
change the angle of the rotor axis from $\theta_{1}=37.38^{\circ}$ to $\theta_{2}=79.19^{\circ}$ was set to $\Delta=$ $100 \mathrm{~ms}$. During this time no noticeable loss of magnetization was observed since the longitudinal relaxation times of both spins are considerably longer than the time $\Delta$. The rf-field strength during the cw decoupling in $\mathrm{t}_{1}$ was $\omega_{1} /(2 \pi) \approx 35.7 \mathrm{kHz}$. After hypercomplex Fourier transformation and phase correction, a slice through the highest point in $\omega_{2}$ along $\omega_{1}$ was taken and is shown in Figure 2.8. A comparison with an MAS spectrum recorded under similar conditions shows a significant narrowing of the line due to the averaging of both the second-rank and fourth-rank tensors. However, the DAS spectrum still has very broad lines, which may be due to inaccuracies in adjusting the two DAS angles or to differences in the cw-decoupling field strengths at the two different rotor orientations (23). The splitting obtained by a fit of the DAS spectrum to two Lorentzian lines is $\Delta \omega=650 \pm 100 \mathrm{~Hz}$. It would be advantageous to implement this experiment under DOR instead of DAS. Both problems, the adjustment of the two angles and the differences in the rf field strengths, would not be present under DOR.

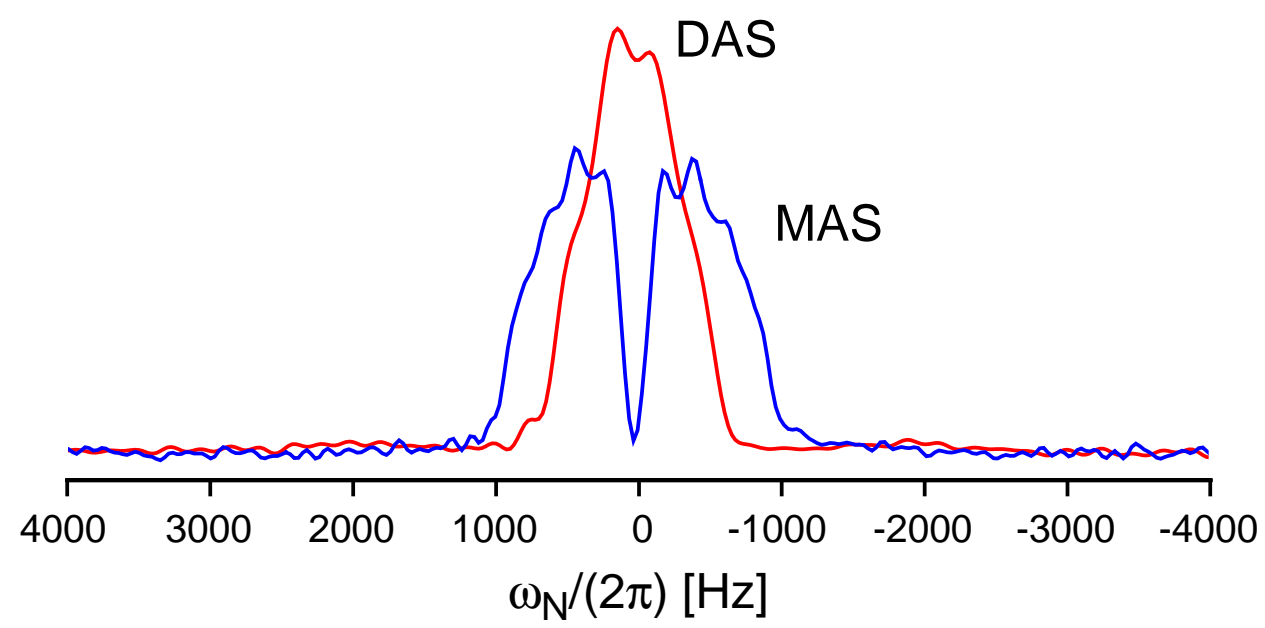

Figure 2.8: Nitrogen magic-angle spinning (MAS) and dynamic-angle spinning (DAS) spectra of $\left[d_{9}\right]$-trimethyl- ${ }^{15} \mathrm{~N}$-ammonium chloride for a decoupling field strength of $\omega_{1} /(2 \pi)=$ $35.7 \mathrm{kHz}$. 


\subsection{References}

1. R.R. Ernst, G. Bodenhausen, and A. Wokaun, Principles of Nuclear Magnetic Resonance in One and Two Dimensions. Clarendon Press, Oxford, 1987.

2. J.R. Sachleben, S. Caldarelli, and L. Emsley, J. Chem. Phys. 104, 2518 (1996).

3. H.J. Reich, M. Jautelat, M.T. Messe, F.J. Weigert, and J.D. Roberts, J. Am. Chem. Soc. 91, 7445 (1969).

4. B. Birdsall, N.J.M. Birdsall, and J Feeney, J. Chem. Soc. Chem. Commun. 1972, 316 (1972).

5. D.M. Brink and G.R. Satchler, Angular Momentum, third edition. Clarendon Press, 1993.

6. M.E. Rose, Elementary Theory of Angular Momentum. Dover, 1995.

7. K.T. Mueller, B.Q. Sun, G.C. Chingas, J.W. Zwanziger, T. Terao, and A. Pines, J. Magn. Reson. 86, 470 (1990).

8. A. Llor and J. Virlet, Chem.Phys.Lett. 152, 248-253 (1988).

9. A. Samoson, E. Lippmaa, and A. Pines, Mol.Phys. 65, 1013 (1988).

10. S. Smith, T. Levante, B.H. Meier, and R.R. Ernst, J. Magn. Reson. Ser. A 106, 75-105 (1994).

11. H.H. Suzukawa, V.B. Cheng, and M. Wolfsberg, J. Chem. Phys. 59, 3992 (1973).

12. D.P. Raleigh, A.C. Kolbert, T.G. Oas, M.H. Levitt, and R.G. Griffin, J.Chem.Soc.,Faraday Trans. 84, 3691 (1988).

13. T.G. Oas, R.G. Griffin, and M.H. Levitt, J. Chem. Phys. 89, 692 (1988).

14. M.H. Levitt, T.G. Oas, and R.G. Griffin, Isr. J. Chem. 28, 271-282 (1988).

15. A.C. Kolbert, D.P. Raleigh, R.G. Griffin, and M.H. Levitt, J. Magn. Reson. 89, 133 (1990).

16. M. Ernst, H. Zimmermann, and B.H. Meier, Chem. Phys. Lett. 317, 581-588 (2000).

17. J. Lindgren and I. Olovsson, Acta. Cryst. B 24, 554 (1968). 
18. M.H. Wann. Solid-State NMR of Liquid Crystals and Biological Molecules. PhD thesis, SUNY at Stony Brook, 1993.

19. M. Ernst, A Verhoeven, and B.H. Meier, J. Magn. Reson. 130, 176-185 (1998).

20. P.J. Grandinetti, J.H. Baltisberger, A. Llor, Y.K. Lee, U. Werner, M.A. Eastman, and A. Pines, J. Magn. Reson. Ser. A 103, 72 (1993).

21. D.J. States, R.A. Haberkorn, and D.J. Ruben, J. Magn. Reson. 48, 286 (1982).

22. M. Ernst, S. Bush, A.C. Kolbert, and A. Pines, J. Chem. Phys. 105, 3387-3397 (1996).

23. M.A. Eastman, P.J. Grandinetti, Y.K. Lee, and A. Pines, J. Magn. Reson. 98, 333 (1992). 


\section{High-Power CW Decoupling in Strongly-Coupled Spin Systems}

\subsection{Theory}

Experimentally, a typical organic solid with strong proton-proton couplings shows relatively sharp lines under $\mathrm{cw}$ decoupling and moderate MAS frequencies (see Fig. 1.4) and not the splitting and broadening predicted by the analysis of an isolated two-spin system (see Eq. [2.15] and Fig. 1.5). Thus, it is obvious that the description of Chapter 2 is not realistic for many spin systems. In rigid organic solids, one is usually faced with an $\mathrm{SI}_{\mathrm{N}}$-spin system where the I spin is not isolated but coupled to a large number of abundant I spins. The dense coupling network of the I spins was neglected in the isolated two-spin system model. It is not clear a priori how many I spins one would have to include before one obtains a realistic picture of a strongly coupled spin system. Hodgkinson et al. (1) estimate that one needs approximately 15 homonuclear spins to reasonably approximate a multi-spin system. An analytical description or a numerical simulation of such a spin system becomes very difficult because the size of the Hilbert space is large, e.g., for a 15-spin system the size of a matrix representation of the Hilbert space is 32768 by 32768 , and numerical simulations are only possible by using special methods. One, therefore, needs a model to include the effects of the strong homonuclear dipolar-coupling network in the simple coherent two-spin model without increasing the dimension of the matrix representation of the density operator.

For labeled samples, one should, in principle, consider an $S_{M} I_{N}$-spin system where the homonuclear couplings between the $S$ spins (homonuclear J and dipolar couplings) are included. Typically the gyromagnetic ratio of the $S$ spins is much lower than that of the I spins $\left(\gamma_{\mathrm{I}} / \gamma_{\mathrm{S}}=4\right.$ for $\mathrm{I}={ }^{1} \mathrm{H}$ and $\mathrm{S}={ }^{13} \mathrm{C}, \gamma_{\mathrm{I}} / \gamma_{\mathrm{S}}=10$ for $\mathrm{I}={ }^{1} \mathrm{H}$ and $\left.S={ }^{15} \mathrm{~N}\right)$. The strength of the homonuclear dipolar-coupling network among the $S$ spins is much weaker since the magnitude of the dipolar couplings depend on the product of the gyromagnetic ratios of the two coupled spins (see Appendix A). Even for moderate MAS frequencies we expect, therefore, that the influence of the 
homonuclear dipolar couplings among the $S$ spins will be averaged out and can usually be neglected. The homonuclear J couplings among the S spins are invariant under MAS and heteronuclear decoupling and will only lead to an additional multiplet structures of the $S$ spin resonances.

One possible model is the description of the strong coupling among the abundant I spins by a spin-diffusion type superoperator, which is isotropic in the Ispin space and leads to spin flips between the $\alpha$ and the $\beta$ states of the I spins (Figure 3.1). Such a superoperator has been used before in the context of transient oscillation in heteronuclear Hartmann-Hahn cross polarization (2). In order to include the spindiffusion superoperator in the description of the spin system, one has to use a Liouville-space representation (3) with the Liouvillian given by:

$$
\hat{\mathscr{L}}(\mathrm{t})=\mathrm{i}[\mathscr{H}(\mathrm{t}), \quad]+\hat{\Gamma}=\mathrm{i} \hat{\mathscr{H}}(\mathrm{t})+\hat{\Gamma}
$$

The Hamiltonian, $\mathscr{H}(\mathrm{t})$, describes the coherent two-spin system and is given by Equation [2.1] while the spin-diffusion superoperator is given by

$$
\hat{\Gamma}=\mathrm{k}_{\mathrm{I}} \cdot\left(\left[\mathrm{I}_{\mathrm{x}},\left[\mathrm{I}_{\mathrm{x}},\right]\right]+\left[\mathrm{I}_{\mathrm{y}},\left[\mathrm{I}_{\mathrm{y}},\right]\right]+\left[\mathrm{I}_{\mathrm{z}},\left[\mathrm{I}_{\mathrm{z}},\right]\right]\right) .
$$

The term spin diffusion in the present context characterizes a generalized polarization-transfer process among the I spins, which is propagated by flip-flop processes. The superoperator of Equation [3.2] is the most simple one since there is

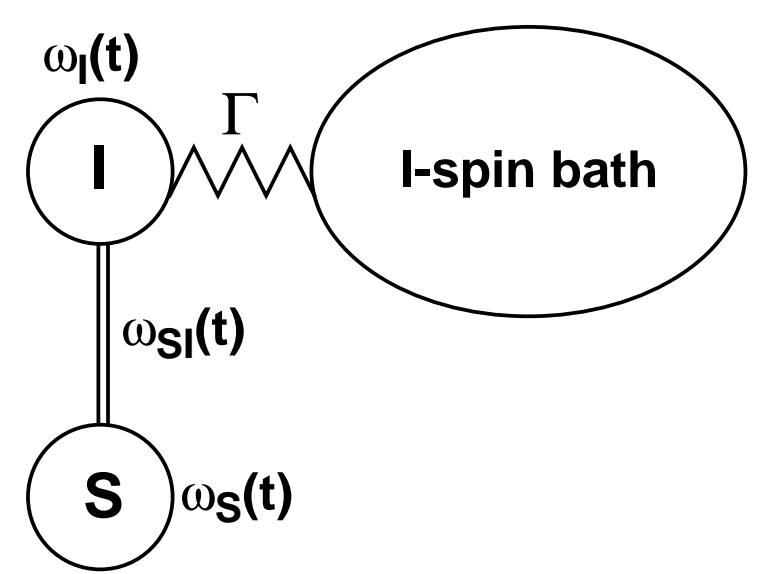

Figure 3.1: Schematic representation of the theoretical model used to simulate heteronuclear spin decoupling. One S spin and a single I spin are described fully quantum mechanically by a Hamiltonian, and the I spin is coupled by a spin-diffusion type superoperator to an I-spin bath. 
only a single rate constant, $\mathrm{k}_{\mathrm{I}}$, that characterizes the polarization-transfer process in the proton bath. One could also use an anisotropic version of $\hat{\Gamma}$ with three rate constants $\mathrm{k}_{\mathrm{x}}, \mathrm{k}_{\mathrm{y}}$, and $\mathrm{k}_{\mathrm{z}}$ but there is no evidence that supports or requires such a complication.

If we calculate the proton decay under the superoperator of Equation [3.2], we find

$$
\left\langle I_{z}\right\rangle(t)=\left\langle I_{z}\right\rangle(0) \cdot e^{-2 k_{I} t}
$$

This relation provides the possibility of experimentally determining the spindiffusion rate constant needed for the model of Equation [3.1].

In the context of the I-spin homonuclear polarization-transfer process, the homonuclear dipolar-coupling Hamiltonian plays the most important role. Under high-power cw irradiation and MAS, the effective homonuclear dipolar-coupling Hamiltonian of Eq. [1.1] is scaled by a factor of $1 / 4$ compared to the effective dipolarcoupling Hamiltonian under MAS alone (4) (see also Eq. [4.4]). The Hamiltonian is scaled by the rf irradiation by $-1 / 2$ due to the second-rank tensor properties of the spin part of the homonuclear dipolar coupling:

$$
\overline{\mathscr{H}}_{\mathrm{II}}^{\operatorname{dip}}(\mathrm{t})=-\frac{1}{2} \cdot \mathscr{H}_{\mathrm{II}}^{\operatorname{dip}}(\mathrm{t})
$$

Magic-angle spinning influences only the spatial part of the homonuclear dipolar coupling. Due to the second-rank tensor properties of the spatial part of the homonuclear dipolar interaction, the zeroth-order average Hamiltonian term vanishes over a full rotor period, $\tau_{\mathrm{r}}=2 \pi / \omega_{\mathrm{r}}$ :

$$
\overline{\mathscr{H}}_{\mathrm{II}}^{(0)}=\frac{1}{\tau_{\mathrm{r}}} \int_{0}^{\tau_{\mathrm{r}}} \overline{\mathscr{H}}_{\mathrm{II}}^{\operatorname{dip}}(\mathrm{t}) \mathrm{dt}=0
$$

The first-order average Hamiltonian term for the homonuclear dipolar interaction $\overline{\mathcal{H}}_{\mathrm{II}}^{\operatorname{dip}}(\mathrm{t})$ is given by 


$$
\begin{aligned}
\overline{\mathscr{H}}_{\mathrm{II}}^{(1)} & =\frac{-\mathrm{i}}{2 \tau_{\mathrm{r}}} \int_{0}^{\tau_{\mathrm{t}}} \mathrm{dt}_{2} \int_{0}^{\mathrm{t}_{2}}\left[\overline{\mathscr{H}}_{\mathrm{II}}^{\mathrm{dip}}\left(\mathrm{t}_{2}\right), \overline{\mathcal{H}}_{\mathrm{II}}^{\mathrm{dip}}\left(\mathrm{t}_{1}\right)\right] \mathrm{dt} \mathrm{t}_{1} \\
& =\frac{1}{4 \omega_{\mathrm{r}}} \sum_{\substack{\mathrm{n} \neq \mathrm{k}, \ell \\
\mathrm{k}<\ell}} \bar{\omega}_{\mathrm{nk} \ell} \cdot\left(4 \mathrm{I}_{\mathrm{nx}} \mathrm{I}_{\mathrm{ky}} \mathrm{I}_{\ell \mathrm{z}}-4 \mathrm{I}_{\mathrm{nx}} \mathrm{I}_{\mathrm{kz}} \mathrm{I}_{\ell \mathrm{y}}\right)
\end{aligned}
$$

where $\bar{\omega}_{\text {nk } \ell}$ depends on the geometry of the dipolar-coupling network (distances and orientation) but not on the MAS rotation frequency. Its full functional form is given in Appendix B. Except for the direction of the quantization axis ( $x$ in the rotating frame, $\mathrm{z}$ in the laboratory frame), we obtain the same functional form of the effective Hamiltonian as with MAS alone (see Eq. [4.4]). While the form of $\overline{\mathscr{H}}_{\text {II }}^{(1)}$ differs from the one of the static dipolar interaction, $\mathscr{H}_{\mathrm{II}}^{\mathrm{dip}}$, it still causes proton flip-flop processes. This type of Hamiltonian can also mediate the same type of polarization transfer as is found in static spin diffusion (4),(5),(6). We expect, therefore, that the spin-diffusion rate constant scales with the inverse of the MAS spinning frequency and that there is a factor of four difference between the laboratory-frame and the rotating-frame spindiffusion rate constants.

By diagonalizing the Liouvillian of Equation [3.1] one can obtain the resonance frequencies and the linewidths of the various transitions. For $\mathrm{k}_{\mathrm{I}}<\left[\left(\omega_{\mathrm{I}}^{\text {iso }}+\omega_{\mathrm{I}}(\mathrm{t})\right) \cdot\left(\pi \mathrm{J}_{\mathrm{SI}}+\omega_{\mathrm{SI}}(\mathrm{t})\right)\right] / \omega_{1}$ we obtain two one-quantum S-spin transitions with the resonance frequencies:

$$
\omega^{(1,2)}(\mathrm{t}) \approx\left(\omega_{\mathrm{S}}^{\text {iso }}+\omega_{\mathrm{S}}(\mathrm{t})\right) \pm \sqrt{\left(\frac{\left(\omega_{\mathrm{I}}^{\text {iso }}+\omega_{\mathrm{I}}(\mathrm{t})\right) \cdot\left(\pi \mathrm{J}_{\mathrm{SI}}+\omega_{\mathrm{SI}}(\mathrm{t})\right)}{\omega_{1}}\right)^{2}-\mathrm{k}_{\mathrm{I}}{ }^{2}}
$$

and the linewidth $\Delta_{1 / 2}^{(1,2)} \approx \mathrm{k}_{\mathrm{I}} / \pi$ (full width at half height). Both resonances have the same intensity. For $\mathrm{k}_{\mathrm{I}}>\left[\left(\omega_{\mathrm{I}}^{\text {iso }}+\omega_{\mathrm{I}}(\mathrm{t})\right) \cdot\left(\pi \mathrm{J}_{\mathrm{SI}}+\omega_{\mathrm{SI}}(\mathrm{t})\right)\right] / \omega_{1}$ we obtain two degenerate lines with the resonance frequency $\omega^{(1,2)} \approx\left(\omega_{S}^{\text {iso }}+\omega_{S}(t)\right)$ and the linewidths:

$$
\Delta_{1 / 2}^{(1,2)}(\mathrm{t}) \approx\left(\mathrm{k}_{\mathrm{I}} \pm \sqrt{\mathrm{k}^{2}-\left(\frac{\left(\omega_{\mathrm{I}}^{\text {iso }}+\omega_{\mathrm{I}}(\mathrm{t})\right) \cdot\left(\pi \mathrm{J}_{\mathrm{SI}}+\omega_{\mathrm{SI}}(\mathrm{t})\right)}{\omega_{1}}\right)^{2}}\right) / \pi
$$


However, the broader of the two resonances has negligible intensity. Equations [3.7] and [3.8] are based on an approximate solution of the eigenvalues of the Liouvillian of Equation [3.1].

Figure 3.2 shows the numerically calculated transition frequencies (Fig. 3.2a) and linewidths (Fig. 3.2b) for an instantaneous time point during MAS in a dipolarcoupled two-spin system as a function of the proton spin-diffusion rate constant, $\mathrm{k}_{\mathrm{I}}$. The data were obtained by numerically diagonalizing the Liouvillian of Equation [3.1] but are in excellent agreement with the approximate solutions of Equations [3.7] and [3.8]. The data shown in Fig. 3.2 were calculated using the parameters $\left(\omega_{\mathrm{I}}^{\text {iso }}+\omega_{\mathrm{I}}(\mathrm{t})\right) /(2 \pi)=2.5 \mathrm{kHz}, \omega_{1} /(2 \pi)=100 \mathrm{kHz}$, and $\left(\omega_{\mathrm{SI}}(\mathrm{t})+\pi \mathrm{J}_{\mathrm{SI}}\right) /(2 \pi)=10 \mathrm{kHz}$ (solid lines), and $20 \mathrm{kHz}$ (dashed lines), respectively. The heteronuclear J coupling and the chemical-shielding tensor of the $S$ spin were assumed to be zero.

a)

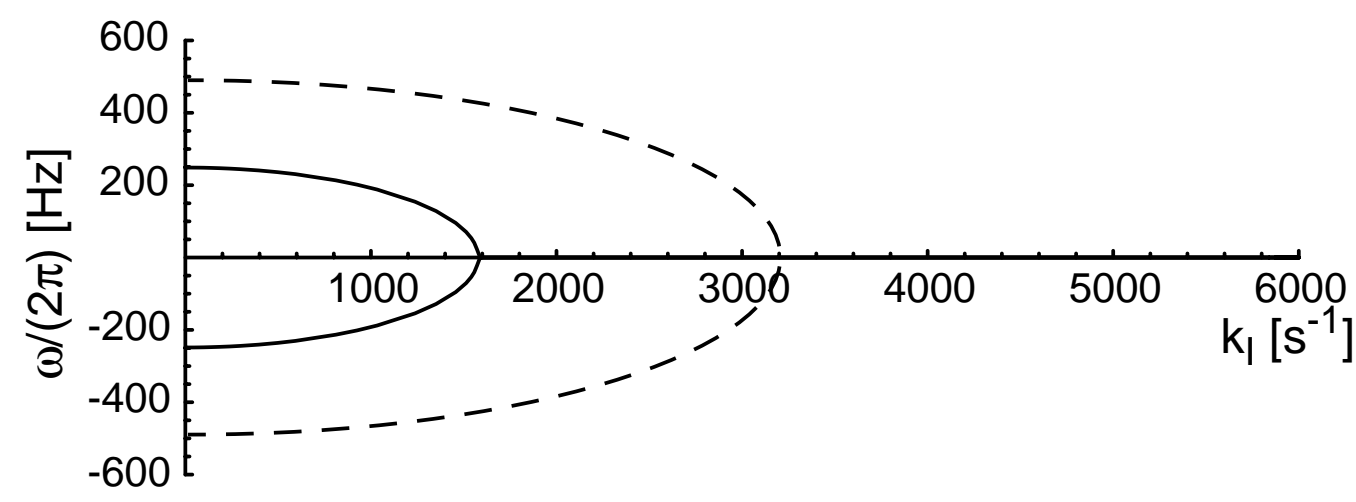

b)

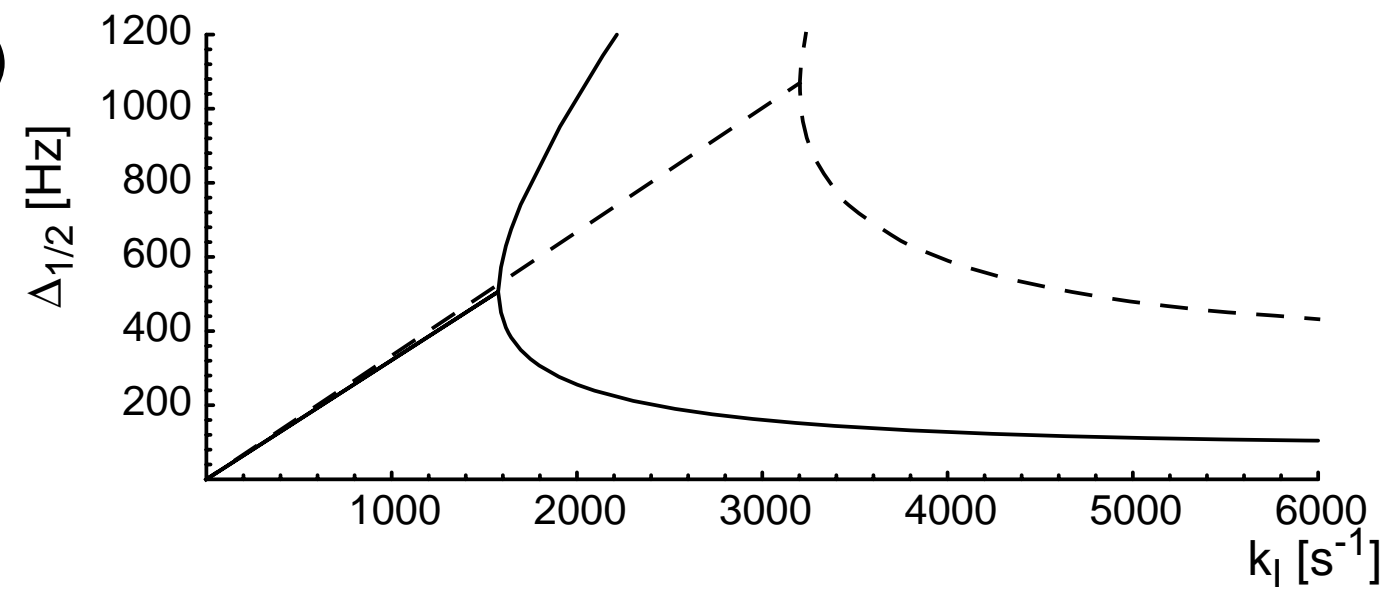

Figure 3.2: (a) Calculated resonance frequencies as a function of the spin-diffusion rate constant $\mathrm{k}_{\mathrm{I}}$. (b) Calculated linewidth $\Delta_{1 / 2}$ (full width at half height) under the same conditions. 
In an MAS experiment, the resonance frequencies, the line widths, and the line intensities are functions of the rotor position and are, therefore, time-dependent. The averaging of these variables over a rotor cycle has no simple analytical solution. In addition we have to perform a powder average over all possible crystallite orientations. In Chapter 3.2, we use numerical Liouville-space simulations to illustrate the line shape and the behavior of the spin system for different values of the spindiffusion rate constant, $\mathrm{k}_{\mathrm{I}}$.

\subsection{Numerical Simulations}

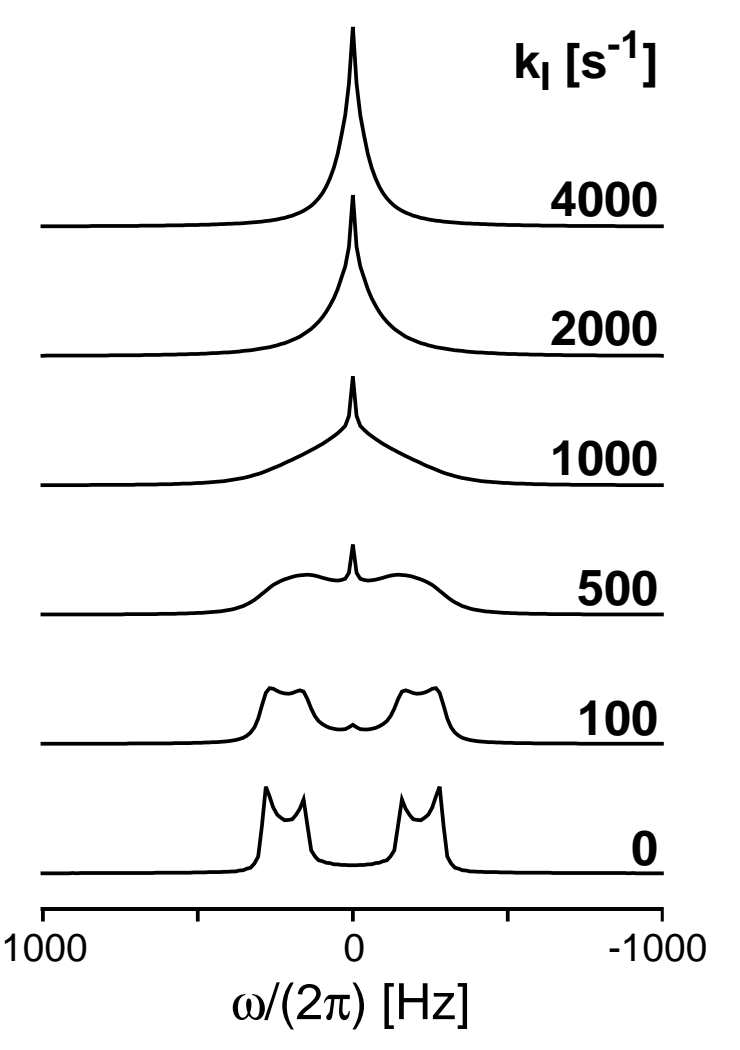

Figure 3.3: Simulated powder line shapes for a two-spin system as a function of the proton spin-diffusion rate constant $\mathrm{k}$
The simulations shown in Figure 3.3 illustrate the behavior of a two-spin system as a function of the I-spin, spin-diffusion rate constant, $\mathrm{k}_{\mathrm{I}}$. All simulations were carried out using the method of small-step, piecewise-constant integration of the Liouville-von-Neumann equation. Figure 3.3 shows the simulated powder patterns for a C-H two-spin system for values of $\mathrm{k}_{\mathrm{I}}$ ranging from $\mathrm{k}_{\mathrm{I}}=0 \mathrm{~s}^{-1}$ to $\mathrm{k}_{\mathrm{I}}=4000 \mathrm{~s}^{-1}$. The dipolar coupling used in the simulations corresponds to a one-bond C-H coupling $\left(\delta_{\mathrm{SI}} /(2 \pi)=46 \mathrm{kHz}\right)$ while the CSA tensor of the I spin was assumed to be parallel to the dipolar coupling with a value of $\Delta \sigma=6 \mathrm{ppm}$ at a proton resonance frequency of $600 \mathrm{MHz}$. The decoupling field strength was set to $\omega_{1} /(2 \pi)=100 \mathrm{kHz}$.

For $\mathrm{k}_{\mathrm{I}}=0 \mathrm{~s}^{-1}$ we obtain the expected two lines for each crystallite orientation in the powder, leading to a fourth-rank powder pattern. With increasing values of $\mathrm{k}$ we see a smoothening of the sharp features of the doublet and the emergence of a sharp line in the center. For $\mathrm{k}_{\mathrm{I}}=4000 \mathrm{~s}^{-1}$ we obtain a single, relatively sharp line at the S-spin 
isotropic shift position with a full width at half height of about $65 \mathrm{~Hz}$. The simulations of Figure 3.3 show that the spin-diffusion model qualitatively describes the expected behavior of a heteronuclear two-spin system. A more quantitative comparison between the model and experimental results will be given in Chapter 3.3.

\subsection{Comparison Between Experiments and Simulations}

To judge the accuracy of the theoretical model described in Chapter 3.1 we have to know the magnitude of the proton spin-diffusion rate constant in order to compare measured spectra with simulations using the spin diffusion model. The proton spin-diffusion rate constant in $8 \%$ labeled $2-{ }^{13} \mathrm{C}$-alanine has been measured following the scheme of local polarization injection (7). A schematic of the pulse sequence used for the measurements is shown in Figure 3.4. It generates localized polarization at protons directly bound to ${ }^{13} \mathrm{C}$ spins. The first cross-polarization $(\mathrm{CP})$ step is only used to enhance the signal intensity. The second and third CP steps are short $\left(\tau_{\mathrm{cp}}=75 \mu \mathrm{s}\right)$ to ensure that the only significant polarization transfer is via onebond proton-carbon dipolar couplings and to avoid proton spin diffusion during the cross-polarization time. During the mixing time, $\tau_{\mathrm{m}}$, the magnetization is either spinlocked (rotating-frame spin-diffusion measurement) or aligned along the z-axis by

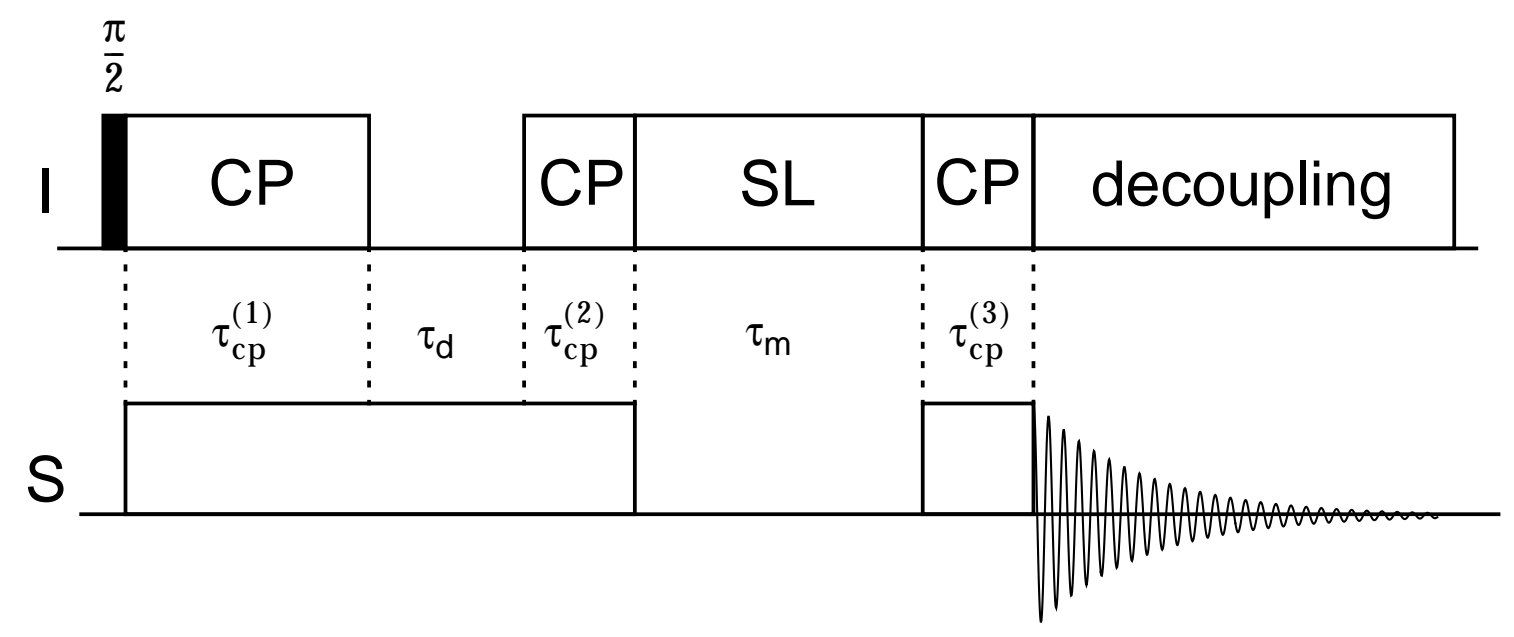

Figure 3.4: Pulse sequence to measure the proton rotating-frame, spin-diffusion rate constant. The second and third cross-polarization time was chosen short to obtain polarization transfer across the one-bond dipolar coupling only. To measure the laboratory-frame spin diffusion rate constant, the spin-lock period (SL) is replaced by a pair of $90^{\circ}$ pulses. 

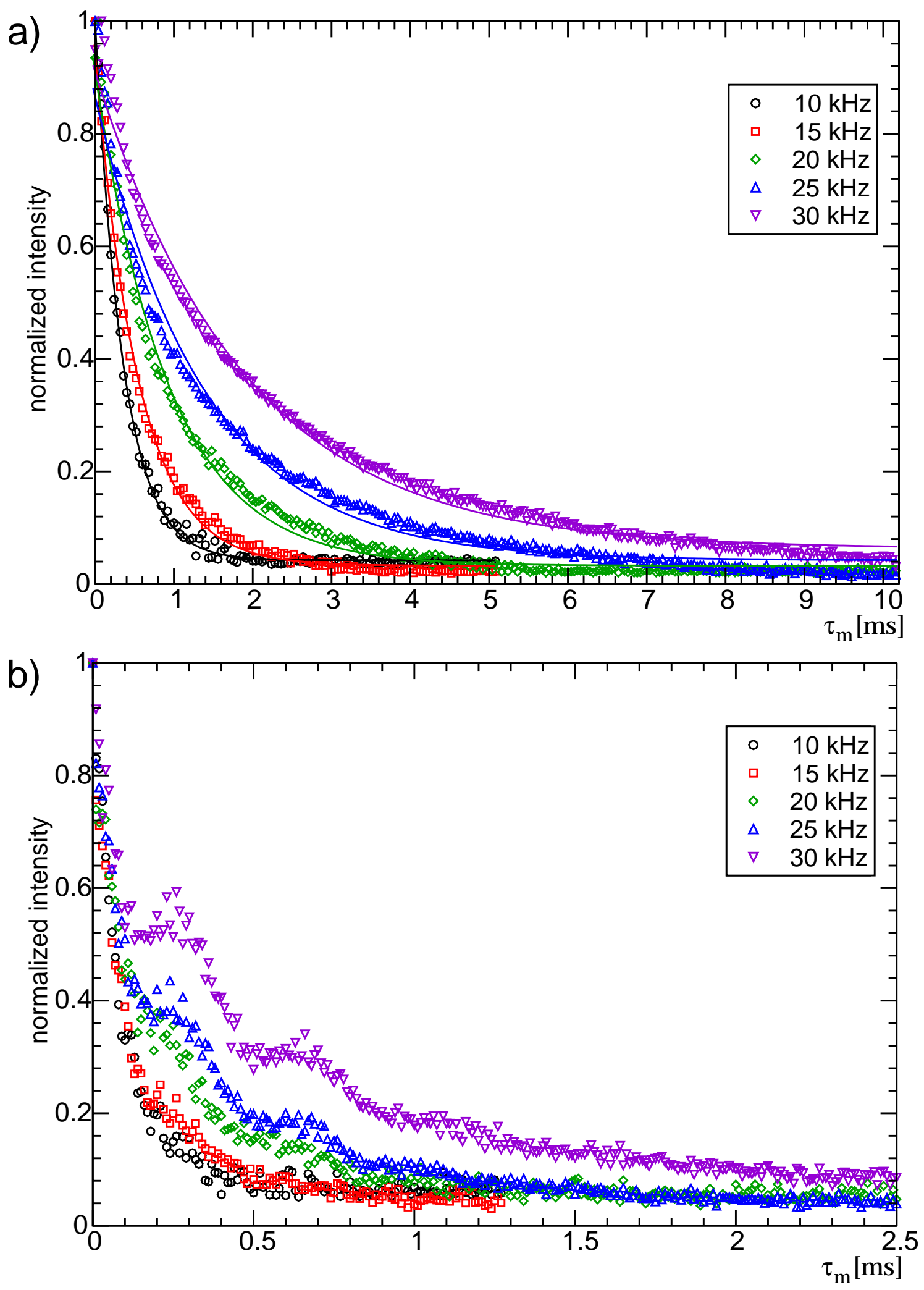

Figure 3.5: Decay of localized proton magnetization in $8 \%$ labeled $2-{ }^{13} \mathrm{C}$-alanine. (a) shows the decay in the rotating frame while (b) shows the decay in the laboratory frame for five different MAS spinning frequencies. 
two $90^{\circ}$ pulses (laboratory-frame spin-diffusion measurement). The intensity of the carbon magnetization is measured as a function of the mixing time, $\tau_{\mathrm{m}}$. Figure 3.5 shows the results of the rotating-frame (Fig. 3.5a) and the laboratory-frame (Fig. 3.5b) measurements. The solid lines in Figure 3.5a show the best mono-exponential fits to the experimental data. One can clearly see that the assumption of a mono-exponential decay was fulfilled to a good approximation. The laboratory-frame measurements of Figure $3.5 \mathrm{~b}$ also show a roughly exponential decay of the polarization. The time constants are about a factor of four larger than the ones obtained from the rotatingframe measurements, as expected from Eq. [3.6]. The source of the additional oscillations on the laboratory-frame measurements is not fully understood.

The measured rate constants, $k_{I}$, are $k_{I}=265 s^{-1}$ for $v_{r}=30 \mathrm{kHz}, k_{I}=360 s^{-1}$ for $v_{\mathrm{r}}=25 \mathrm{kHz}, \mathrm{k}_{\mathrm{I}}=540 \mathrm{~s}^{-1}$ for $v_{\mathrm{r}}=20 \mathrm{kHz}, \mathrm{k}_{\mathrm{I}}=920 \mathrm{~s}^{-1}$ for $v_{\mathrm{r}}=15 \mathrm{kHz}$, and $\mathrm{k}_{\mathrm{I}}=1400 \mathrm{~s}^{-1}$ for $v_{\mathrm{r}}=10 \mathrm{kHz}$. Figure 3.6 shows the measured spin-diffusion rate constants as a function of the spinning frequency. As expected, the rate constants corresponding to spinning frequencies in the range between 10 and $30 \mathrm{kHz}$ are approximately proportional to the inverse of the spinning frequency as predicted by Equations [3.6] and [4.4]. These

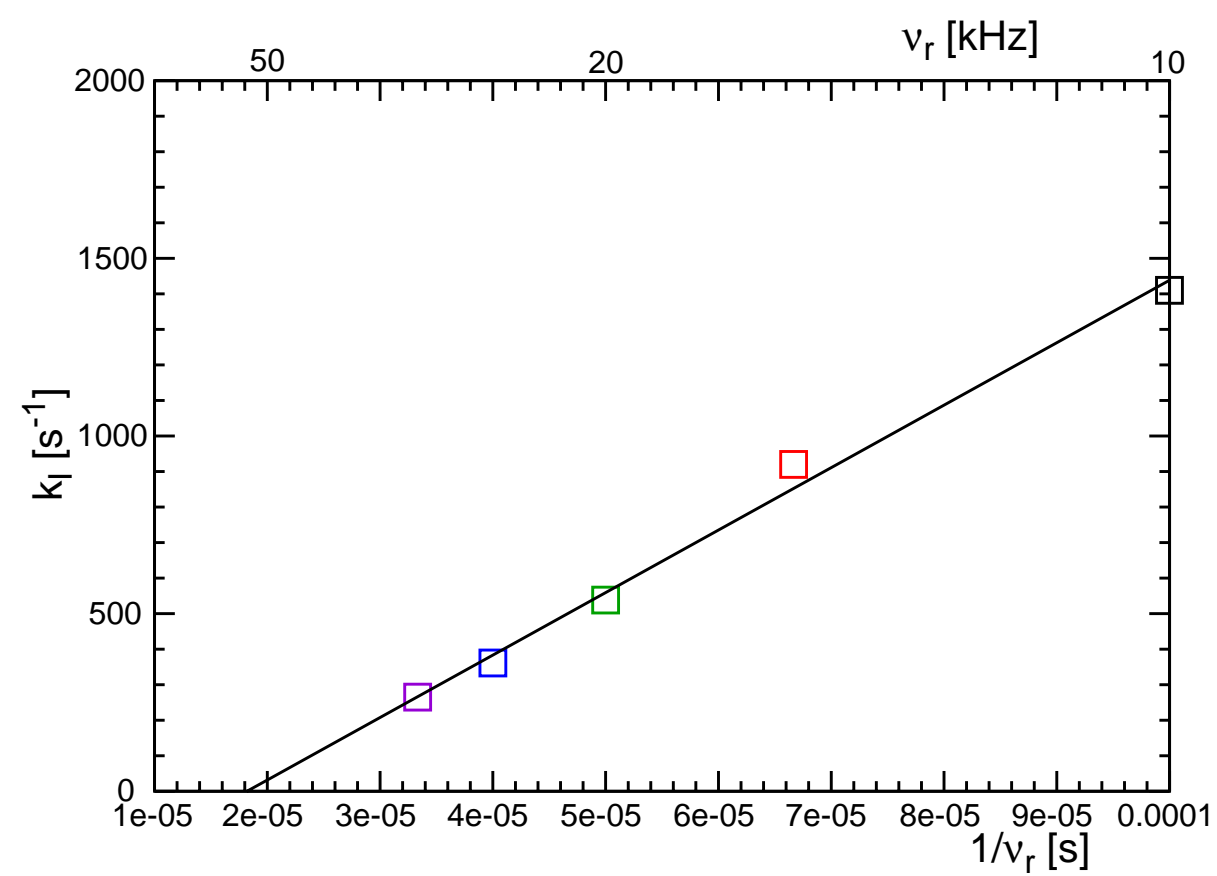

Figure 3.6: Measured rotating-frame, spin-diffusion rate constants in $8 \%$ labeled $2-{ }^{13} \mathrm{C}$-alanine as a function of the MAS frequency. 
rate constants can be used to simulate $\mathrm{cw}$ decoupled spectra of alanine and to compare the simulations with experimental data.

Figure 3.7a shows ${ }^{13} \mathrm{C}$ spectra of $2-{ }^{13} \mathrm{C}$-alanine at spinning frequencies ranging from $10 \mathrm{kHz}$ to $30 \mathrm{kHz}$ measured at a proton resonance frequency of $600 \mathrm{MHz}$ and with a cw decoupling field strength of $\omega_{1} /(2 \pi)=100 \mathrm{kHz}$. It can clearly be seen that the linewidth of the line increases with increasing spinning frequency. Such a spinning-frequency dependent line broadening has been observed experimentally before at lower spinning frequencies $(8),(9)$ in samples with partially averaged dipolar
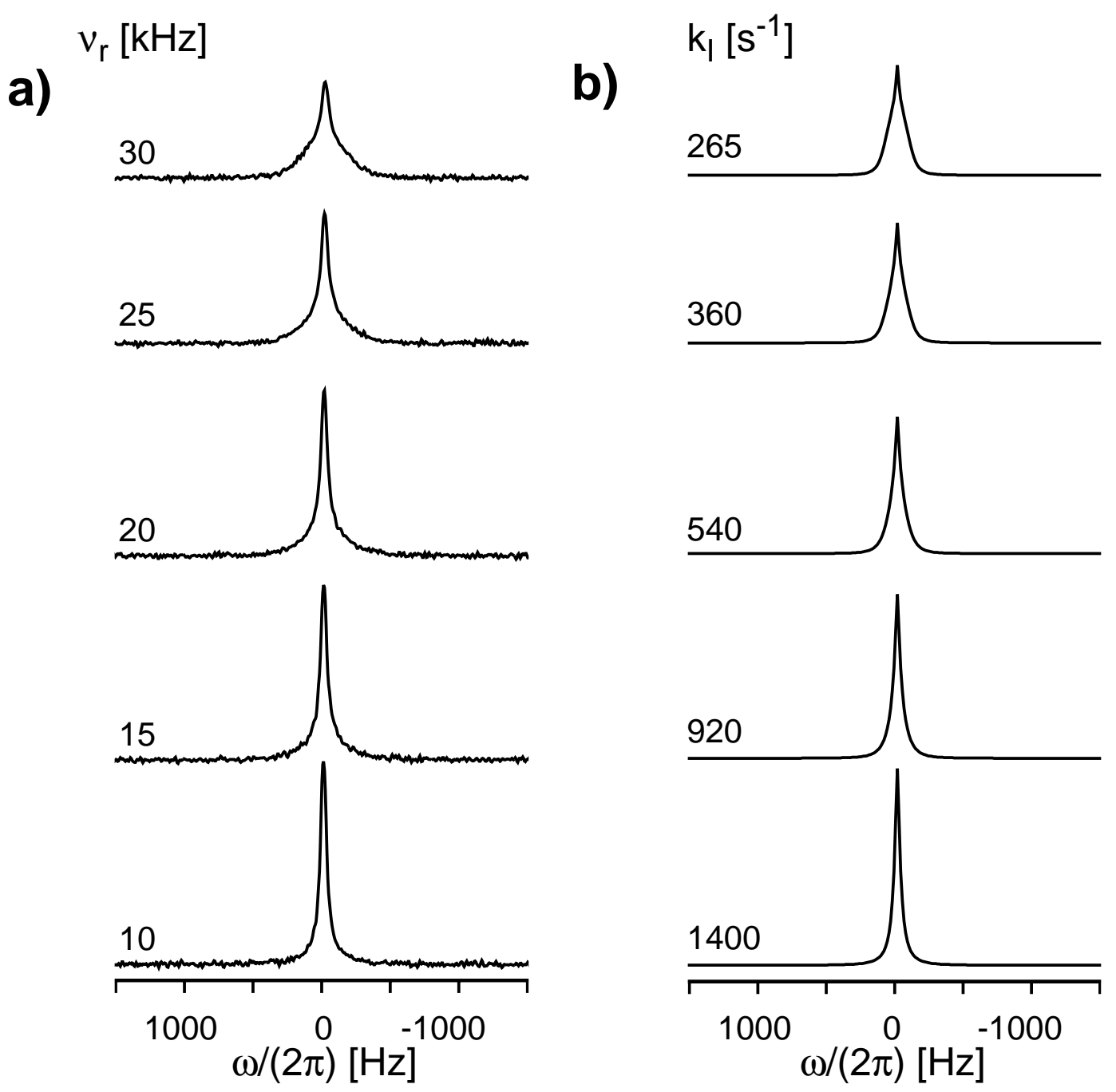

Figure 3.7: (a) Measured spectra of $2-{ }^{13} \mathrm{C}$-alanine using a $\mathrm{cw}$ decoupling field strength of $\omega_{1} /(2 \pi)=100 \mathrm{kHz}$ at different spinning frequencies between $v_{\mathrm{r}}=10 \mathrm{kHz}$ and $30 \mathrm{kHz}$ at a proton resonance frequency of $600 \mathrm{MHz}$. The simulated spectra in (b) use the measured spin-diffusion rate constants, $\mathrm{k}_{\mathrm{I}}$, and assume a one-bond $\mathrm{C}-\mathrm{H}$ dipolar coupling. 
couplings. At lower spinning frequencies the line shape appears to be described quite well by a mixed Gaussian/Lorentzian line while at higher spinning frequencies the line shape becomes more complicated and seems to have several components. The simulated spectra in Figure 3.7b were obtained using the measured proton spindiffusion rate constants and assuming a one-bond $\mathrm{C}-\mathrm{H}$ dipolar coupling and an axially symmetric proton chemical-shielding tensor of $\Delta \sigma=4 \mathrm{ppm}$. The agreement between the simulated and the measured spectra is quite good, keeping in mind that there was only a single adjustable parameter for the set of simulations, namely the chemical-shielding tensor of the protons. This indicates that the model of Chapter 3.1 provides a good theoretical framework for the description of strongly-coupled spin systems under high-power cw irradiation.

\subsection{References}

1. P. Hodgkinson, S. Dimitris, and L. Emsley, Chem. Phys. Lett. 326, 515-522 (2000).

2. L. Muller, A. Kumar, T. Baumann, and R.R. Ernst, Phys. Rev. Lett. 32, 1402 (1974).

3. R.R. Ernst, G. Bodenhausen, and A. Wokaun, Principles of Nuclear Magnetic Resonance in One and Two Dimensions. Clarendon Press, Oxford, 1987.

4. M. Ernst, A Verhoeven, and B.H. Meier, J. Magn. Reson. 130, 176-185 (1998).

5. C. Filip, X. Filip, D.E. Demco, and S. Hafner, Mol. Phys. 92, 757-771 (1997).

6. C. Filip, S. Hafner, I. Schnell, D.E. Demco, and H.W. Spiess, J. Chem. Phys. 110, 423-440 (1999).

7. S. Zhang, B.H. Meier, and R.R. Ernst, Solid State NMR 1, 313-320 (1992).

8. I.J. Shannon, K.D.M. Harris, and S. Arumugan, Chem.Phys.Lett. 196, 588-594 (1992).

9. P. Tekely, P. Palmas, and D. Canet, J. Magn. Reson. A 107, 129-133 (1994). 


\section{Low-Power CW Decoupling}

\subsection{Theory}

As already mentioned earlier, in the case of low-power cw decoupling (1) the order of the averaging processes is reversed compared to high-power cw decoupling under MAS. The rotation of the sample about the magic angle is now the faster of the two averaging processes (see Figure 1.3b). The MAS time-averaged Hamiltonian can be calculated from the time-dependent Hamiltonian of Eq. [1.1] using average Hamiltonian theory (2). If the spinning frequency approaches infinity, the zerothorder average Hamiltonian provides an exact description of the time evolution of the spin system. The Hamiltonian is then given by

$$
\begin{aligned}
\overline{\mathscr{H}}^{(0)} & =\frac{1}{\tau_{\mathrm{r}}} \cdot \int_{0}^{\tau_{\mathrm{r}}} \mathrm{d} \mathrm{t} \cdot \mathscr{H}(\mathrm{t}) \\
& =\mathscr{H}_{\mathrm{S}}^{\mathrm{CS}}+\mathscr{H}_{\mathrm{I}}^{\mathrm{CS}}+\mathscr{H}_{\mathrm{II}}^{\mathrm{J}}+\mathscr{H}_{\mathrm{SI}}^{\mathrm{J}} .
\end{aligned}
$$

This Hamiltonian contains only the first four terms of Eq. [1.1]. The Hamiltonian of Eq. [4.1] is the usual liquid-state NMR Hamiltonian.

For finite MAS frequencies, we have to consider higher-order terms in the average Hamiltonian expansion. The first-order average Hamiltonian is given by

$$
\overline{\mathscr{H}}^{(1)}=\frac{-\mathrm{i}}{2 \tau_{\mathrm{r}}} \cdot \int_{0}^{\tau_{\mathrm{r}}} \mathrm{dt}_{2} \cdot \int_{0}^{\mathrm{t}_{2}} \mathrm{dt}_{1} \cdot\left[\mathscr{H}\left(\mathrm{t}_{2}\right), \mathscr{H}\left(\mathrm{t}_{1}\right)\right] .
$$

Analyzing the commutators in Eq. [4.2] is straightforward and leads, in the absence of rf irradiation, to only three non-vanishing terms: (i) the cross term between the homonuclear dipolar coupling of the I spins with the CSA tensor of the I spins

$$
\overline{\mathscr{H}}_{\mathrm{a}}^{(1)}=\frac{1}{\omega_{\mathrm{r}}} \cdot \sum_{\mathrm{k}<\ell} \bar{\omega}_{\mathrm{k} \ell} \cdot\left(2 \mathrm{I}_{\mathrm{kx}} \mathrm{I}_{\ell \mathrm{y}}-2 \mathrm{I}_{\mathrm{ky}} \mathrm{I}_{\ell \mathrm{x}}\right) ;
$$

(ii) the cross term of the homonuclear dipolar coupling of the I spins with itself 


$$
\overline{\mathscr{H}}_{\mathrm{b}}^{(1)}=\frac{1}{\omega_{\mathrm{r}}} \cdot \sum_{\substack{\mathrm{n} \neq \mathrm{k}, \ell \\ \mathrm{k}<\ell}} \bar{\omega}_{\mathrm{nk} \ell} \cdot\left(4 \mathrm{I}_{\mathrm{nz}} \mathrm{I}_{\mathrm{kx}} \mathrm{I}_{\ell \mathrm{y}}-4 \mathrm{I}_{\mathrm{nz}} \mathrm{I}_{\mathrm{ky}} \mathrm{I}_{\ell \mathrm{x}}\right) ;
$$

and (iii) the cross term between the homonuclear dipolar coupling of the I spins and the heteronuclear dipolar coupling

$$
\overline{\mathscr{H}}_{\mathrm{c}}^{(1)}=\frac{1}{\omega_{\mathrm{r}}} \cdot \sum_{\mathrm{k}<\ell} \bar{\omega}_{\mathrm{Sk} \ell} \cdot\left(4 \mathrm{~S}_{\mathrm{z}} \mathrm{I}_{\mathrm{kx}} \mathrm{I}_{\ell \mathrm{y}}-4 \mathrm{~S}_{\mathrm{z}} \mathrm{I}_{\mathrm{ky}} \mathrm{I}_{\ell \mathrm{x}}\right) \text {. }
$$

This term leads to a direct broadening of the $S$ spin resonances since it has the form of a generalized coupling between the $S$ spin and the I spins. The effective coupling frequencies $\bar{\omega}_{\mathrm{Sk} \ell}$ can be calculated from the commutators of Eq. [4.2] and are given explicitly in Appendix B.

The first two contributions act only indirectly on the S-spin resonances by propagating spin diffusion among the I spins (3),(4). We assume that the MAS spinning frequency is high enough that we can neglect terms that depend on $\left(1 / \omega_{\mathrm{r}}\right)^{2}$ and higher powers in the average Hamiltonian expansion. This assumption is fulfilled to a good approximation for spinning frequencies larger than $10 \mathrm{kHz}$ as can be seen from the good fit of the expected $1 / \omega_{\mathrm{r}}$ dependence of the line width to the measured line widths in undecoupled spectra of a $\mathrm{CH}$ and $\mathrm{CH}_{2}$ group (see Figure 1.2).

Based on the three terms of Eqs. [4.3]-[4.5], which form the first-order average Hamiltonian, two potential sources for the residual linewidth under high-frequency MAS without proton irradiation become apparent: (i) The spin diffusion among the I spins mediated by the Hamiltonians of Eqs. [4.3] and [4.4] can lead to an "exchangetype" narrowing or broadening of the J-multiplet (3),(4). (ii) The generalized heteronuclear coupling terms of Eq. [4.5] can lead to a direct broadening of the S-spin resonances. 
We can estimate the maximum contribution to the residual linewidth generated by spin diffusion acting on a heteronuclear J-splitting. Figure 4.1 shows carbon spectra simulated for a J-coupled $\mathrm{CH}$ two-spin system where the spin diffusion among the protons is included in the simulation using the model presented in Chapter 3.1 (3). The J-coupling was set to $130 \mathrm{~Hz}$, a typical value for a $\mathrm{CH}$ group. The strength of the coupling in the I-spin bath was varied. The maximum linewidth obtained was about $90 \mathrm{~Hz}$ FWHH at a value of $\mathrm{k}_{\mathrm{I}}=500 \mathrm{~s}^{-1}$. This clearly indicates that at the spinning frequencies considered here a major source for the residual linewidth is the cross term between the homonuclear and the heteronuclear dipolar couplings (Eq. [4.5]) in the first-order average Hamiltonian.

Adding the rf-irradiation term

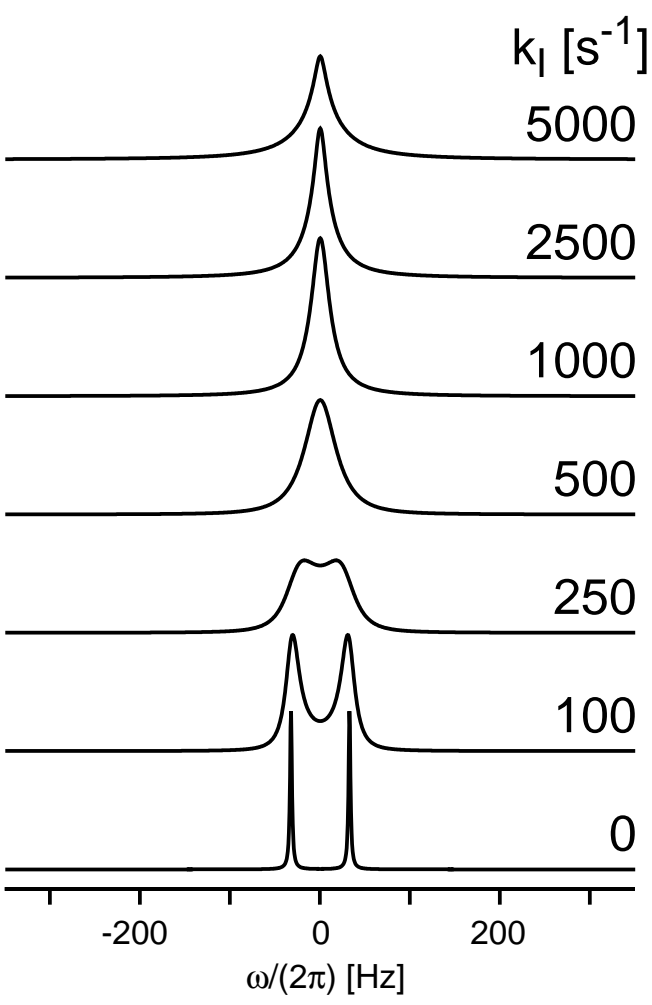

Figure 4.1: Simulation of an isolated dipolar-coupled $\mathrm{CH}$ two-spin system with a J-coupling of $130 \mathrm{~Hz}$ under MAS. The coupling of the two-spin system to the proton bath was described by a relaxation superoperator. The parameter $\mathrm{k}_{\mathrm{I}}$ which was varied in the simulations is a measure for the coupling strength in the proton bath.

$\left(\mathscr{H}^{\mathrm{rf}}=\sum_{\mathrm{k}} \omega_{1} \mathrm{I}_{\mathrm{kx}}\right)$ to the Hamiltonian leads to

three additional important terms in the first-order average Hamiltonian of Eq. [4.2]. These three terms are (i) the cross term between the rf irradiation and the chemicalshielding tensor of the I spins

$$
\overline{\mathscr{H}}_{\mathrm{d}}^{(1)}=\frac{\omega_{1}}{\omega_{\mathrm{r}}} \cdot \sum_{\mathrm{k}} \bar{\omega}_{\mathrm{k}}^{\mathrm{rf}} \cdot \mathrm{I}_{\mathrm{ky}}
$$

(ii) the cross term between the rf irradiation and the heteronuclear dipolar coupling tensor

$$
\overline{\mathcal{H}}_{\mathrm{e}}^{(1)}=\frac{\omega_{1}}{\omega_{\mathrm{r}}} \cdot \sum_{\mathrm{k}} \bar{\omega}_{\mathrm{Sk}}^{\mathrm{rf}} \cdot 2 \mathrm{~S}_{\mathrm{z}} \mathrm{I}_{\mathrm{ky}} ;
$$


and (iii) the cross term between the rf irradiation and the homonuclear dipolar coupling tensor

$$
\overline{\mathscr{H}}_{\mathrm{f}}^{(1)}=\frac{\omega_{1}}{\omega_{\mathrm{r}}} \cdot \sum_{\mathrm{k}<\ell} \bar{\omega}_{\mathrm{k} \ell}^{\mathrm{rf}} \cdot\left(2 \mathrm{I}_{\mathrm{ky}} \mathrm{I}_{\ell \mathrm{z}}+2 \mathrm{I}_{\mathrm{kz}} \mathrm{I}_{\ell \mathrm{y}}\right)
$$

The averaged constants $\bar{\omega}_{\mathrm{k}}^{\mathrm{rf}}, \bar{\omega}_{\mathrm{Sk}}^{\mathrm{rf}}$, and $\bar{\omega}_{\mathrm{k} \ell}^{\mathrm{rf}}$ can be calculated from the commutators of Eq. [4.2] and are given in Appendix B.

Assuming that the rf-irradiation field strength is much lower than the spinning frequency but considerably larger than the residual dipolar coupling after averaging by MAS we can now transform the MAS-averaged Hamiltonian into a interaction frame quantized along the rf field and perform a second averaging step. The total Hamiltonian before this second averaging step is given by

$$
\overline{\mathscr{H}}=\overline{\mathscr{H}}^{(0)}+\overline{\mathscr{H}}_{\mathrm{a}}^{(1)}+\overline{\mathscr{H}}_{\mathrm{b}}^{(1)}+\overline{\mathscr{H}}_{\mathrm{c}}^{(1)}+\overline{\mathscr{H}}_{\mathrm{d}}^{(1)}+\overline{\mathscr{H}}_{\mathrm{e}}^{(1)}+\overline{\mathscr{H}}_{\mathrm{f}}^{(1)} .
$$

If we only consider zeroth-order average Hamiltonian terms in this interaction frame by the cw rf field we obtain a Hamiltonian

$$
\overline{\overline{\mathscr{H}}}^{(0)}=\mathscr{H}_{\mathrm{S}}^{\mathrm{CS}}+\mathscr{H}_{\mathrm{II}}^{\mathrm{J}}+\frac{1}{2 \omega_{\mathrm{r}}} \cdot \sum_{\substack{\mathrm{n} \neq \mathrm{k}, \ell \\ \mathrm{k}<\ell}} \bar{\omega}_{\mathrm{nk} \ell}^{\prime} \cdot\left(4 \mathrm{I}_{\mathrm{nx}} \mathrm{I}_{\mathrm{ky}} \mathrm{I}_{\ell \mathrm{z}}-4 \mathrm{I}_{\mathrm{nx}} \mathrm{I}_{\mathrm{kz}} \mathrm{I}_{\ell \mathrm{y}}\right)
$$

which contains only the isotropic chemical shift of the S spins, the homonuclear J coupling of the I spins, and a term originating from the homonuclear dipolar couplings of the I spins. The last term of Eq. [4.10] promotes spin diffusion among the I spins and has a similar structure to the one found when employing high-power cw decoupling (4) (see also Eq. [3.6]). The constant $\bar{\omega}_{\text {nk } \ell}^{\prime}$ can be calculated from the interaction-frame transformation and is given in Appendix B.

The system is only described to a good approximation by the Hamiltonian of Eq. [4.10] if the rf field is considerably larger than the I-spin terms in $\overline{\mathscr{H}}$. Especially critical are the isotropic chemical-shift offsets of the I spins, the heteronuclear J coupling, and the first-order terms involving rf irradiation. On the other hand, the consecutive averaging approach is only valid if the rf field is considerably smaller than the spinning frequency. Therefore we expect the best results at high spinning 
frequencies since this will lead to smaller terms in $\overline{\mathscr{H}}^{(1)}$ and, at the same time, allows the use of higher "low-power" decoupling fields without interference effects from the sample spinning.

If we assume that $\omega_{\mathrm{r}}=\mathrm{p} \cdot \omega_{1}$, we obtain the following first-order average Hamiltonian where only terms linear in $1 / \omega_{\mathrm{r}}$ and involving the $\mathrm{S}$-spin operators are shown:

$$
\begin{aligned}
\overline{\overline{\mathscr{H}}}(1)= & \sum_{\mathrm{k}}\left(\frac{\mathrm{p} \omega_{\mathrm{k}} \pi \mathrm{J}_{\mathrm{Sk}}}{\omega_{\mathrm{r}}}+\frac{\bar{\omega}_{\mathrm{Sk}}^{\mathrm{rf}} \bar{\omega}_{\mathrm{k}}^{\mathrm{rf}}}{\mathrm{p} \omega_{\mathrm{r}}}\right) \cdot 2 \mathrm{~S}_{\mathrm{z}} \mathrm{I}_{\mathrm{kz}}+ \\
& \sum_{\mathrm{k}<\ell} \frac{\pi \mathrm{J}_{\mathrm{k} \ell} \cdot\left(\bar{\omega}_{\mathrm{S} \ell}^{\mathrm{rf}}-\bar{\omega}_{\mathrm{Sk}}^{\mathrm{rf}}\right)}{\omega_{\mathrm{r}}} \cdot\left(4 S_{\mathrm{z}} \mathrm{I}_{\mathrm{kx}} \mathrm{I}_{1 \mathrm{y}}-4 S_{\mathrm{z}} \mathrm{I}_{\mathrm{ky}} \mathrm{I}_{1 \mathrm{x}}\right)
\end{aligned}
$$

It is interesting to note that again the cross terms between the chemical-shielding tensor of the I spins and the heteronuclear dipolar-coupling tensor dominates the first-order average Hamiltonian as was found in the case of high-power decoupling.

We can also calculate the average Hamiltonian by simultaneously averaging over the rotation of the spin part and the space part of the Hamiltonian assuming that the ratio between the rf-irradiation field strength and the spinning frequency is an integer. The zeroth-order average Hamiltonian is given as

$$
\overline{\mathscr{H}}^{(0)}=\mathscr{H}_{\mathrm{S}}^{\mathrm{CS}}+\mathscr{H}_{\mathrm{II}}^{\mathrm{J}} \text {, }
$$

assuming that $\mathrm{p}>2$, i.e., no recoupling condition (e.g., HORROR condition at $\left.\omega_{1}=\omega_{\mathrm{r}} / 2\right)$ is matched. The first-order average Hamiltonian for a ratio $\omega_{\mathrm{r}}=\mathrm{p} \cdot \omega_{1}$, where $\mathrm{p}$ is an integer, is given by:

$$
\begin{aligned}
\overline{\mathscr{H}}^{(1)}= & \sum_{\mathrm{k}}\left[\frac{\mathrm{p} \omega_{\mathrm{k}} \pi \mathrm{J}_{\mathrm{Sk}}}{\omega_{\mathrm{r}}}+\frac{\mathrm{p}-4 \mathrm{p}^{3}}{1-5 \mathrm{p}^{2}+4 \mathrm{p}^{4}} \frac{\omega_{\mathrm{Sk}}^{+1} \omega_{\mathrm{k}}^{-1}+\omega_{\mathrm{Sk}}^{-1} \omega_{\mathrm{k}}^{+1}}{\omega_{\mathrm{r}}}\right. \\
& \left.+\frac{\mathrm{p}-\mathrm{p}^{3}}{1-5 \mathrm{p}^{2}+4 \mathrm{p}^{4}} \frac{\omega_{\mathrm{Sk}}^{+2} \omega_{\mathrm{k}}^{-2}+\omega_{\mathrm{Sk}}^{-2} \omega_{\mathrm{k}}^{+2}}{\omega_{\mathrm{r}}}\right] \cdot 2 \mathrm{~S}_{\mathrm{z}} \mathrm{I}_{\mathrm{kx}}
\end{aligned}
$$

Only terms involving S-spin operators are shown for simplicity. For large values of $\mathrm{p}$ we can simplify Eq. [4.13] and obtain: 


$$
\overline{\mathscr{H}}^{(1)}=\sum_{\mathrm{k}}\left[\frac{\mathrm{p} \omega_{\mathrm{k}} \pi \mathrm{J}_{\mathrm{Sk}}}{\omega_{\mathrm{r}}}-\sum_{\substack{\mathrm{m}=-2 \\ \mathrm{~m} \neq 0}}^{2} \frac{\omega_{\mathrm{Sk}}^{+\mathrm{m}} \omega_{\mathrm{k}}^{-\mathrm{m}}}{\mathrm{m}^{2} \mathrm{p} \omega_{\mathrm{r}}}\right] \cdot 2 \mathrm{~S}_{\mathrm{z}} \mathrm{I}_{\mathrm{kx}}
$$

which contains similar terms as one obtains from the sequential averaging approach in Eq. [4.11]. One has to keep in mind that for large values of $p$ the cycle time of the average Hamiltonian calculation becomes quite long and the question of the convergence of the average Hamiltonian approach has to be considered.

It is interesting to compare this average Hamiltonian to the case of high-power cw irradiation as shown in Eq. [2.19]. Exactly the same terms dominate the residual line width, and only the weighting prefactors, which depend on the ratio $\mathrm{p}$ between the spinning frequency and the rf-field strength, have a different functional form.

There are two possible ways to further decrease the linewidth using the lowpower rf-irradiation approach. One can further increase the MAS frequency and, thereby, reduce the size of the first-order terms in Eqs. [4.13] and [4.14]. Increasing the spinning frequency will also allow the use of higher rf-irradiation fields which will decrease the size of the first-order average Hamiltonian even more. The second approach would be the design of multiple-pulse sequences which reduce the size of the first-order terms. Here one has to avoid recoupling conditions which could, potentially, lead to an unwanted broadening of the lines.

\subsection{Numerical Simulations}

Numerical simulations of low-power cw decoupling suffer from the same problems as numerical simulation of high-power cw decoupling. Simulating a small spin system does not reflect the reality of a strongly coupled proton system and one has to find ways to incorporate the effect of strong proton couplings into a small coherently simulated spin system.

Nevertheless, numerical simulations in small spin systems can be used to illustrate the fact that the cross term between the chemical-shielding tensor and the heteronuclear dipolar coupling tensor is indeed the dominant contribution to the 
residual line width for low-power cw decoupling. Figure 4.2 shows the maximum line intensity for a three-spin simulation in a C-H-H system at an MAS spinning frequency of $50 \mathrm{kHz}$ as a function of the rf-field strength. The spin system consists of a dipolar coupled $\mathrm{CH}$ pair with $\delta_{\mathrm{CH}} /(2 \pi)=46 \mathrm{kHz}$ and an additional proton which is dipolar coupled to the first proton only $\left(\delta_{\mathrm{HH}} /(2 \pi)=40 \mathrm{kHz}\right)$. Without proton irradiation the line width is, as expected from Eq. [4.5], dominated by the presence of a large homonuclear dipolar coupling. Setting the homonuclear dipolar coupling to zero (and keeping the rf irradiation off) leads to an intensity of one independent of the size of the proton CSA tensor. For the whole range of rf-field strengths, the addition of a CSA tensor to the proton which is coupled to the carbon $\left(\delta_{\mathrm{H}} /(2 \pi)=3 \mathrm{kHz}, \eta_{\mathrm{H}}=0\right.$, coaxial with the dipolar coupling) leads to a significant reduction in the maximum line

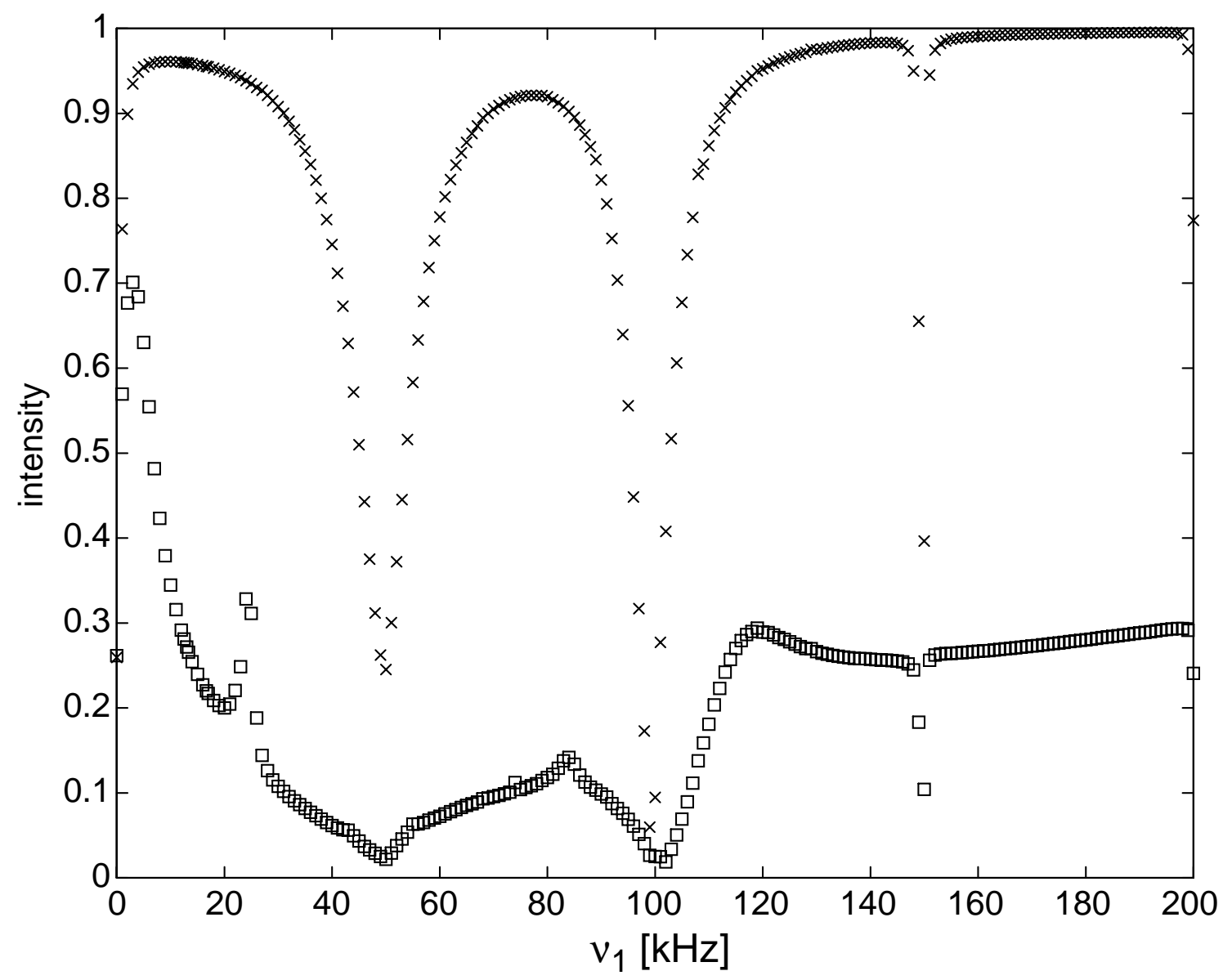

Figure 4.2: Maximum peak intensity of a simulated three-spin system under MAS rotation $\left(v_{\mathrm{r}}\right.$ $=50 \mathrm{kHz})$ as a function of the rf-field strength. The crosses $(x)$ indicate the values for simulations without a CSA tensor on the protons, the squares $(\square)$ indicate values for simulations including a CSA tensor on the proton coupled to the carbon $\left(\delta_{\mathrm{H}} /(2 \pi)=3 \mathrm{kHz}\right.$, $\eta_{\mathrm{H}}=0$, coaxial with the dipolar coupling). 
intensity. This is in good agreement with Eqs. [2.18] and [4.13] which predict that the cross term between the chemical-shielding tensor and the heteronuclear dipolar coupling tensor dominates the residual line width for both high-power and lowpower cw decoupling. The simulations without the CSA tensor show almost perfect decoupling, i.e., an intensity close to one, over the whole range of rf-field strengths except when the $n=1,2,3$, or 4 rotary-resonance conditions $\left(\omega_{1}=n \omega_{r}\right)(5),(6),(7),(8)$ are matched. One can also see that matching the HORROR condition $\left(\omega_{1}=\omega_{\mathrm{r}} / 2\right)$ in the case of a non-negligible CSA tensor leads to an increased line intensity due to a recoupling of the homonuclear dipolar coupling.

\subsection{Experimental Results and Discussion}

All experiments presented in this section were carried out on a Bruker DMX500 spectrometer equipped with a $1.8 \mathrm{~mm}$ o.d. double-resonance MAS probe capable of spinning up to $50 \mathrm{kHz}$ (9). In order to accurately calibrate the decoupler field strengths, the amplitude of the rf-pulses was measured by attenuating the output of the amplifier and feeding it into the receiver of the NMR system. The obtained voltage is a measure of the rf-field strength. The calibration of the voltage in terms of frequency units was then carried out by measuring the nutation frequency at a few selected power levels (Figure 4.3)

To evaluate the decoupling performance as a function of the rf-field strength at different spinning frequencies, experiments were performed on $8 \%$ labeled $2-{ }^{13} \mathrm{C}$ alanine (Figure 4.4) and on 5\% labeled $2-{ }^{13} \mathrm{C}$-glycine (Figure 4.6). Enriched samples were used to enhance the rate of data collection. The degree of labeling used in the experiments does not influence the results presented here. The alanine sample was used to characterize the behavior of a $\mathrm{CH}$ group while the glycine sample was used to characterize a $\mathrm{CH}_{2}$ group. The spinning frequency was varied between 20 and $50 \mathrm{kHz}$ and the rf-field strength was varied between 1 and $215 \mathrm{kHz}$ in steps of $0.1 \mathrm{~dB}$. For each spinning frequency and decoupling field strength a $1 \mathrm{D}{ }^{13} \mathrm{C}$ spectrum was recorded, and the line width and the line intensity were obtained by fitting the FID with a single mixed Lorentzian/Gaussian line. The assumption of such a line shape 
proved to be good. The value for the full width at half height (FWHH) was obtained by fitting the FID and extracting the numerical values for the FWHH from the best fit.

Figure 4.4 shows the line width of the ${ }^{13} \mathrm{C}$ line in alanine $(\mathrm{FWHH})$ in a $\mathrm{cW}$ decoupling experiment as a function of the ratio of the decoupler field strength and the spinning frequency for the four spinning frequencies $v_{\mathrm{r}}=20,30,40$, and $50 \mathrm{kHz}$. It can clearly be seen that the line becomes broad around the well-known rotaryresonance conditions $n \cdot v_{r}=v_{1}$ with $n=1,2(6),(7)$ where the heteronuclear dipolar coupling is recoupled due to the interference between the spinning frequency and the rf-field strength. As expected, weak rotary-resonance recoupling for $n>2$ is also observed. The HORROR recoupling condition (10) at $\frac{1}{2} \cdot v_{\mathrm{r}}=\mathrm{v}_{1}$ is not observed in these measurements because HORROR recouples only the homonuclear dipolar couplings, which do not significantly influence the line width of the ${ }^{13} \mathrm{C}$ line.

At the highest decoupling field strength $\left(v_{1}=215 \mathrm{kHz}\right.$, last data point in all measurements) we find that the line width increases significantly with increasing spinning frequency as expected (11). This increase in line width is due to the closer proximity to the $n=2$ rotary-resonance condition and to the reduction of the effective homonuclear dipolar coupling among the protons at higher spinning frequencies. The

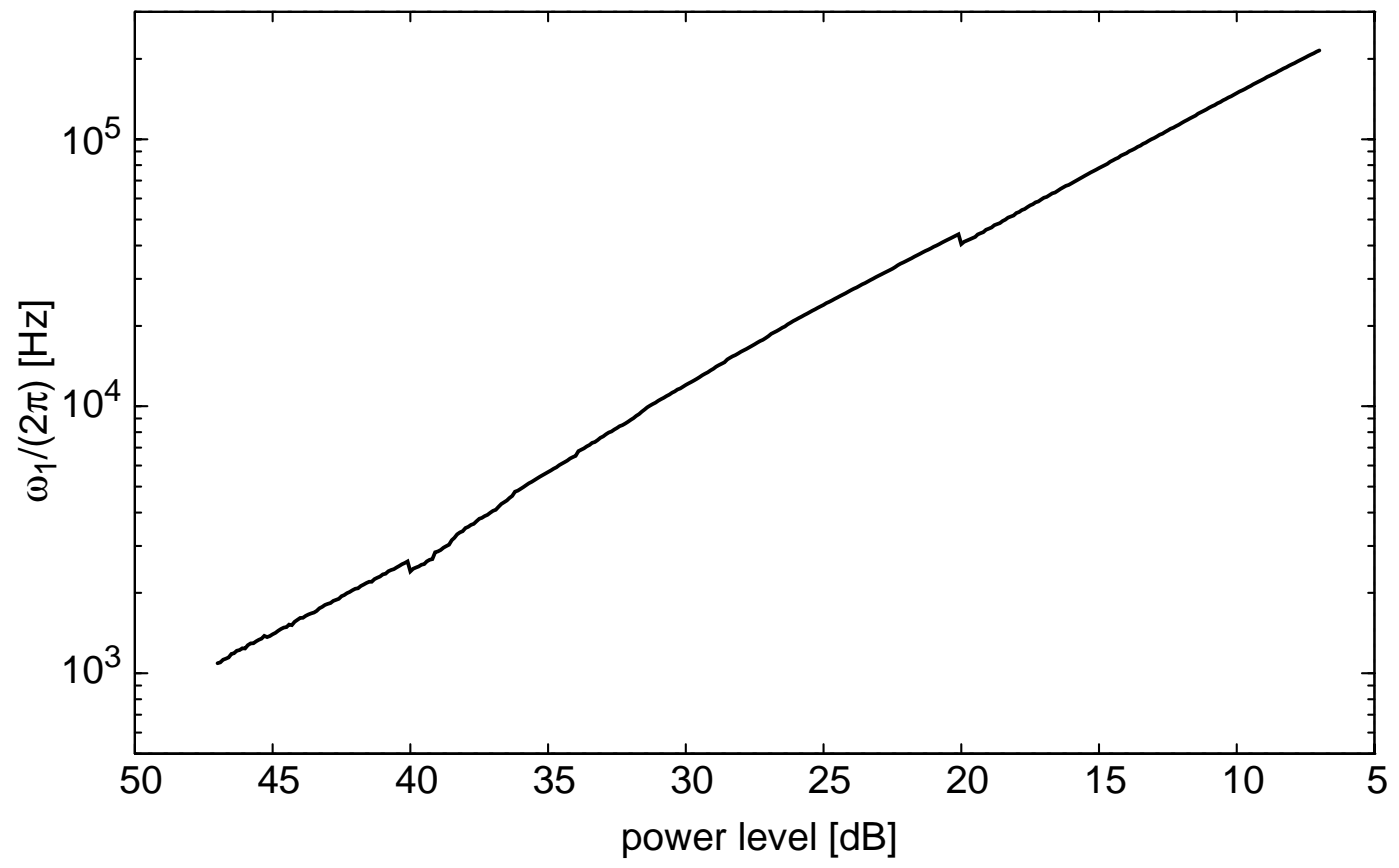

Figure 4.3: RF-field strength calculated from the measured voltage as a function of the powerlevel setting of the spectrometer. 
reduced proton-proton coupling strength leads to a quenching of the spin diffusion and thus to a reduced "self-decoupling" (3),(12),(13),(14) of the residual line broadening after cw decoupling (15). At $20 \mathrm{kHz}$ MAS spinning frequency the line width obtained at $215 \mathrm{kHz}$ decoupling field strength is about $40 \mathrm{~Hz}$ while at $50 \mathrm{kHz}$ MAS the residual line width is above $60 \mathrm{~Hz}$.

If we look at decoupling field strengths below the $n=1$ rotary-resonance condition, we find that at the higher spinning frequencies it is possible to achieve reasonably good decoupling. At $20 \mathrm{kHz}$ MAS the narrowest line width obtained is $300 \mathrm{~Hz}$ FWHH at a decoupling field strength of about $5 \mathrm{kHz}$. At $30 \mathrm{kHz}$ MAS the line width is reduced to $70 \mathrm{~Hz}$ at a decoupling field strength of $8.5 \mathrm{kHz}$, and at $40 \mathrm{kHz}$ the line width is only about $50 \mathrm{~Hz}$ at an rf-field strength of $10 \mathrm{kHz}$. Under these conditions, the line is already narrower than the one obtained with a cw decoupling

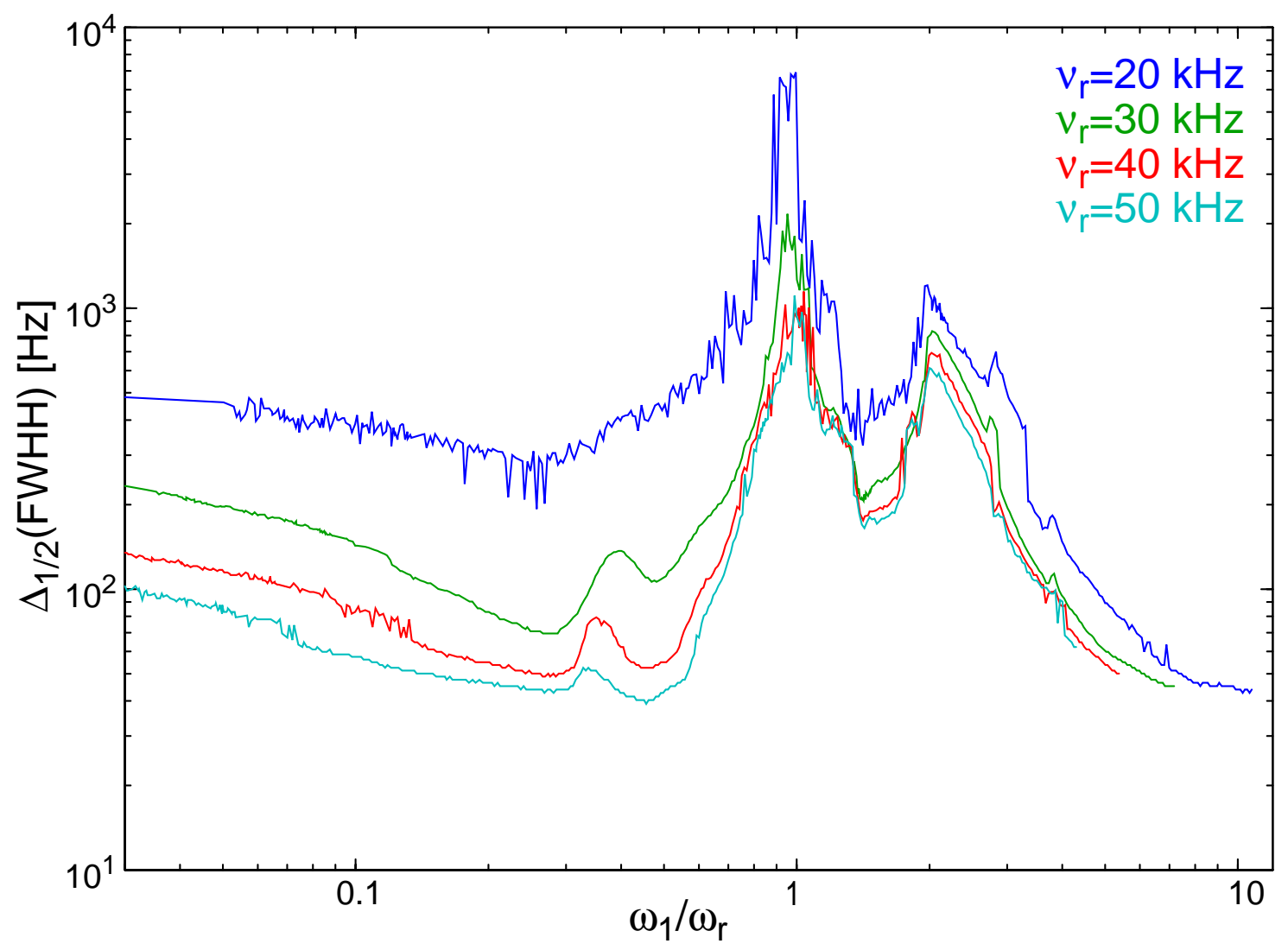

Figure 4.4: ${ }^{13} \mathrm{C}$ line width $(\mathrm{FWHH})$ in $8 \%$ labeled $2-{ }^{13} \mathrm{C}$-alanine at MAS spinning frequencies of $20,30,40$, and $50 \mathrm{kHz}$ as a function of the decoupler field strength scaled by the MAS frequency. 
field of $215 \mathrm{kHz}$ at the same spinning frequency. At $50 \mathrm{kHz}$ MAS the narrowest line width of about $40 \mathrm{~Hz}$ is obtained at a field strength of $21 \mathrm{kHz}$. This width is quite close to the minimum of $35 \mathrm{~Hz}$ obtained in this sample using TPPM decoupling at $45 \mathrm{kHz}$ MAS and an rf-field strength of $215 \mathrm{kHz}$ (Figure 4.5c).

To give an impression of the quality of the spectra, some low-power decoupled spectra for the four MAS spinning frequencies are shown in Figure 4.5b. The decoupling power was set to the value in the interval from 0 to half the spinning frequency that gives the lowest line width in Fig. 4.4. Figure 4.5a shows the spectra at the same MAS frequencies for high-power decoupling $\left(v_{1}=215 \mathrm{kHz}\right)$, and Figure 4.5c shows an optimized TPPM-decoupled spectrum at an MAS frequency of $45 \mathrm{kHz}$ and an rf-field strength of $215 \mathrm{kHz}$. All spectra are shown on the same scale to allow direct comparison of line intensity and line width.

Figures 4.6 shows the dependence of the line width on the rf-field strength in cw decoupled spectra for a $\mathrm{CH}_{2}$ group in $2-{ }^{13} \mathrm{C}$-glycine. In principle, the same

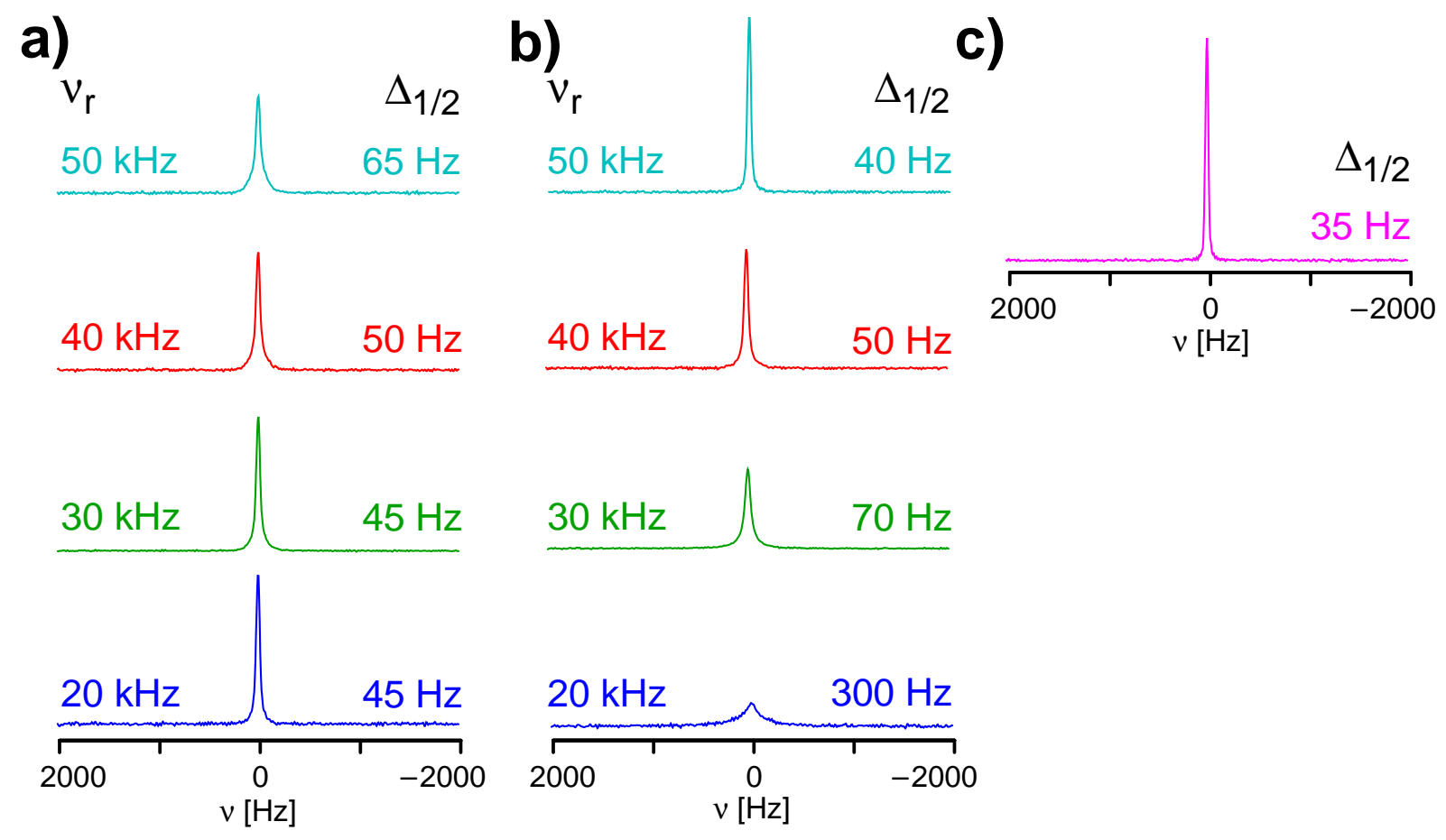

Figure 4.5: a) High-power decoupled spectra obtained with a cw-decoupling field strength of $215 \mathrm{kHz}$; b) optimized low-power $\mathrm{cw}$-decoupled spectra of $2-{ }^{13} \mathrm{C}$-alanine $(\mathrm{CH})$ at four spinning frequencies; c) optimized TPPM decoupled spectrum at $45 \mathrm{kHz}$ MAS spinning frequency. 
features as in the $\mathrm{CH}$ group are observed, but the overall line width is increased significantly. This illustrates the well-known fact that $\mathrm{CH}_{2}$ groups are generally more difficult to decouple than $\mathrm{CH}$ groups due to the strong homonuclear dipolar coupling of about $\delta_{\mathrm{HH}} /(2 \pi)=40 \mathrm{kHz}$. The higher-order rotary-resonance conditions are much more pronounced than in the measurement of the $\mathrm{CH}$ group (Figure 4.4). The narrowest lines for low-power decoupling are found to be about $60 \mathrm{~Hz}$ FWHH. They are obtained at $50 \mathrm{kHz}$ MAS frequency and a decoupling field strength of about $11 \mathrm{kHz}$. This width is about $20 \%$ larger than the $50 \mathrm{~Hz}$ obtained in this sample using TPPM decoupling at $215 \mathrm{kHz}$ with $45 \mathrm{kHz}$ MAS (Figure 4.7c). Figure 4.7 shows again the best low-power cw decoupled spectra (Figure 4.7b) in comparison with the best high-power cw decoupled spectra (Figure 4.7a) to allow direct comparison of the line intensities and the line widths obtained by the different methods. For higher MAS spinning frequencies, low-power cw decoupling is again superior to high-power cw

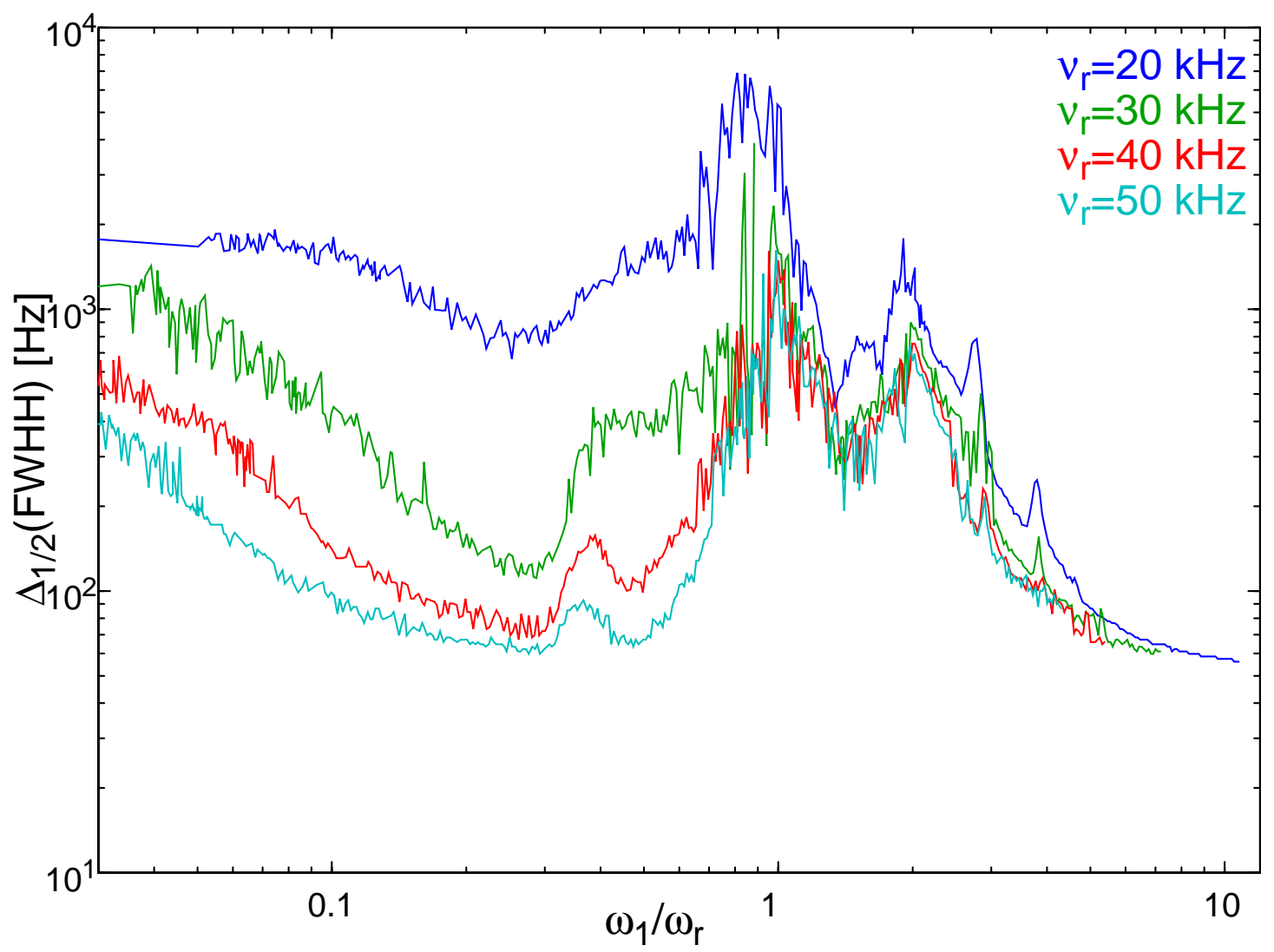

Figure 4.6: ${ }^{13} \mathrm{C}$ line width $(\mathrm{FWHH})$ in $5 \%$ labeled $2-{ }^{13} \mathrm{C}$-glycine at MAS spinning frequencies of $20,30,40$, and $50 \mathrm{kHz}$ as a function of the decoupler field strength scaled by the MAS spinning frequency. 
decoupling, but the narrowest lines are still obtained using optimized TPPM decoupling.

The bandwidth of cw decoupling at low rf-field strengths is, of course, limited and an accurate setting of the rf-irradiation frequency is important. Simple offsetcompensating composite pulse sequences such as WALTZ-4 (16),(17) gave only very small improvements in the bandwidth compared to $\mathrm{cw}$ irradiation. Since the dominant term for the residual line width under low-power decoupling (Eq. [4.11]) has the same structure as the one under high-power decoupling (Eq. [2.19]), one can expect that further improvements in low-power decoupling are possible by using pulse sequences that reduce the size of the first-order average Hamiltonian term.

To illustrate the performance of the decoupling with low-power $\mathrm{cw}$ irradiation in a larger molecule, tests were performed on a sample of a fully ${ }^{13} \mathrm{C}$ and ${ }^{15} \mathrm{~N}$ labeled cyclic decapeptide, antamanide, which can be crystallized to yield quite narrow lines (18). Figure 4.8 shows spectra of antamanide (a) at $50 \mathrm{kHz}$ and (b) at $30 \mathrm{kHz}$ MAS

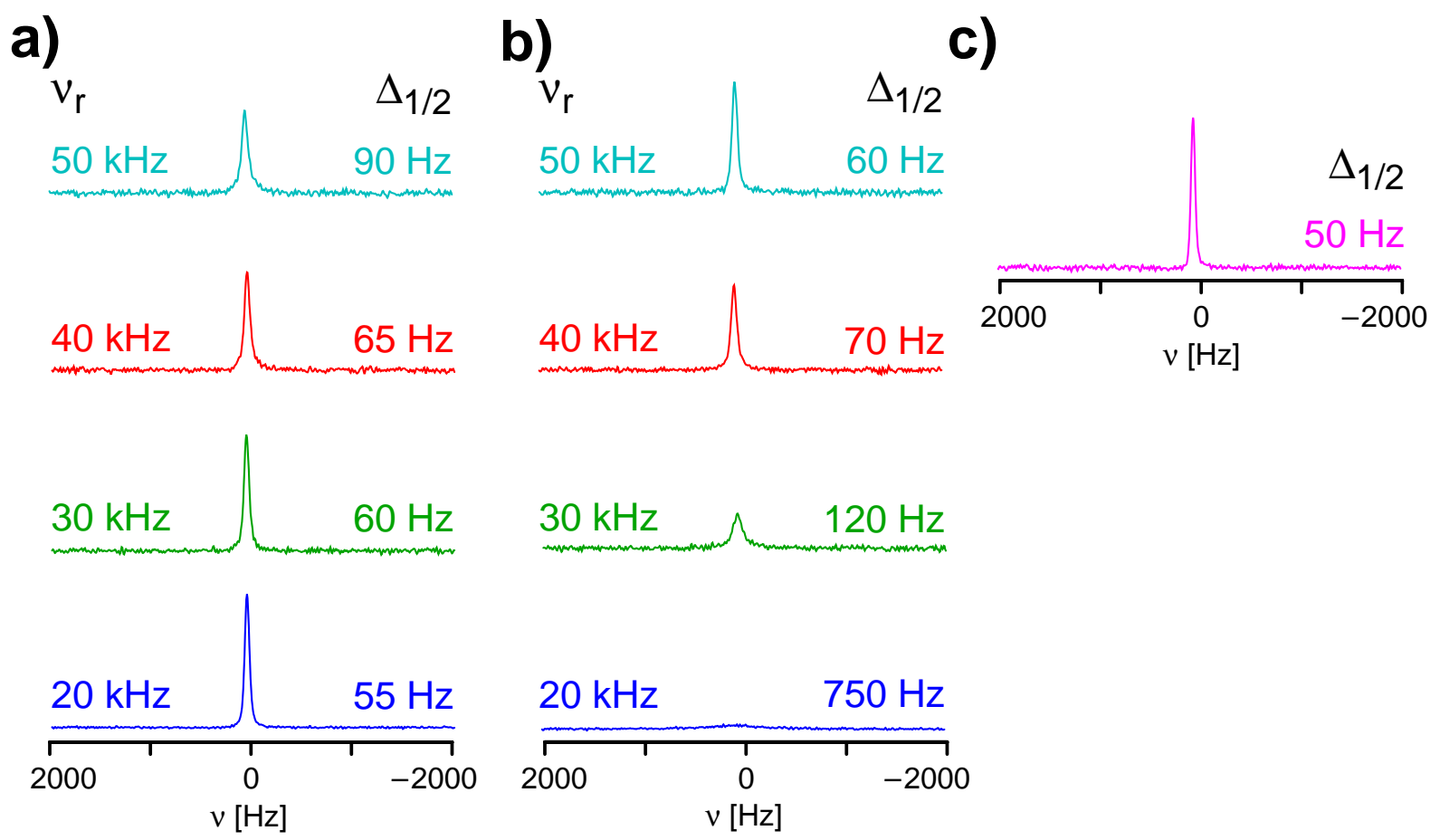

Figure 4.7: a) High-power decoupled spectra obtained with a cw-decoupling field strength of $215 \mathrm{kHz}$; b) optimized low-power cw-decoupled spectra of ${ }^{2-}{ }^{13} \mathrm{C}$-glycine $\left(\mathrm{CH}_{2}\right)$ at four spinning frequencies; c) optimized TPPM decoupled spectrum at $45 \mathrm{kHz}$ MAS spinning frequency. 
frequencies. At $50 \mathrm{kHz}$ MAS frequency (Fig. 4.8a) low-power cw decoupling shows a significant improvement over high-power cw decoupling using an rf-field strength of $185 \mathrm{kHz}$. It is, however, also obvious that optimized TPPM decoupling can reduce the line width even further. In the chemical-shift range of the $\alpha$-carbons (approximately 51-62 ppm) the differences between the $10 \mathrm{kHz} \mathrm{cw}$ decoupled spectrum and the TPPM decoupled spectra are relatively small, and similar line widths are obtained in

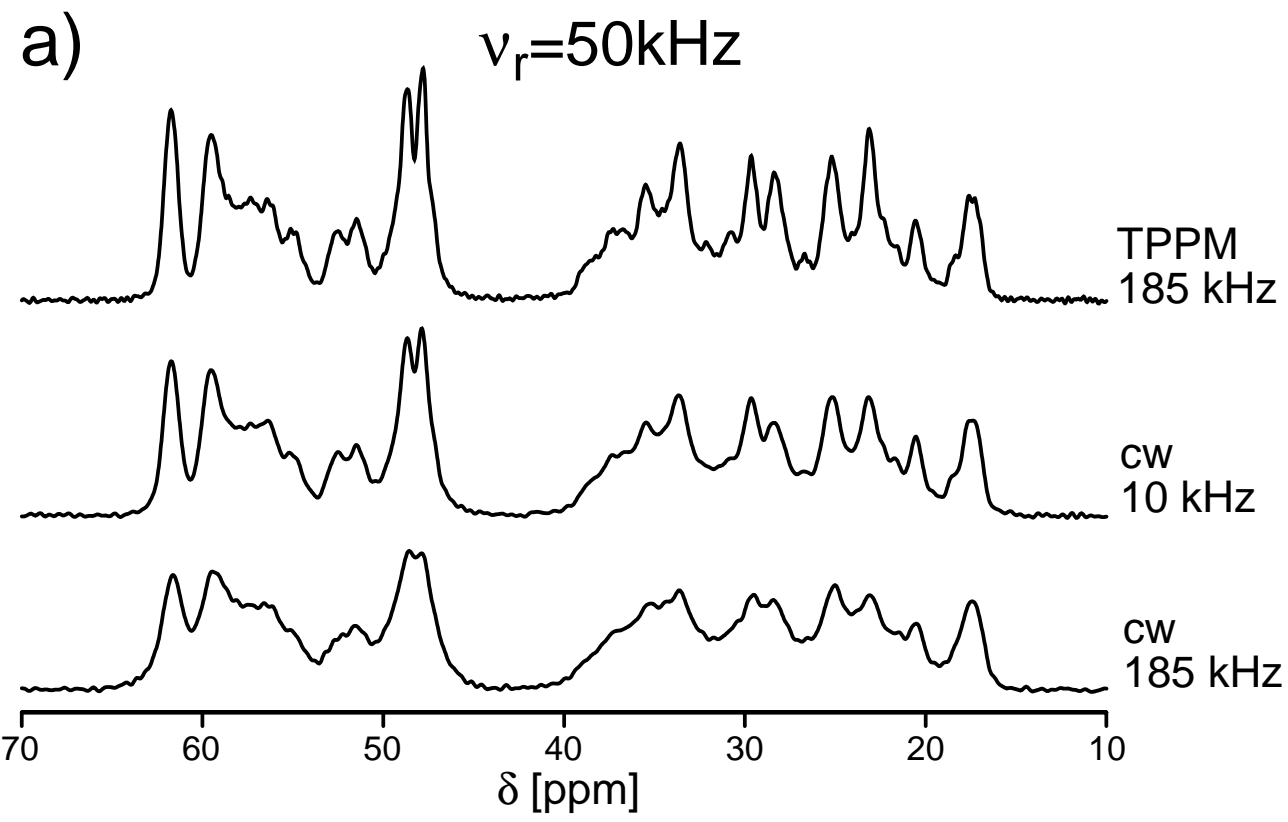

b)
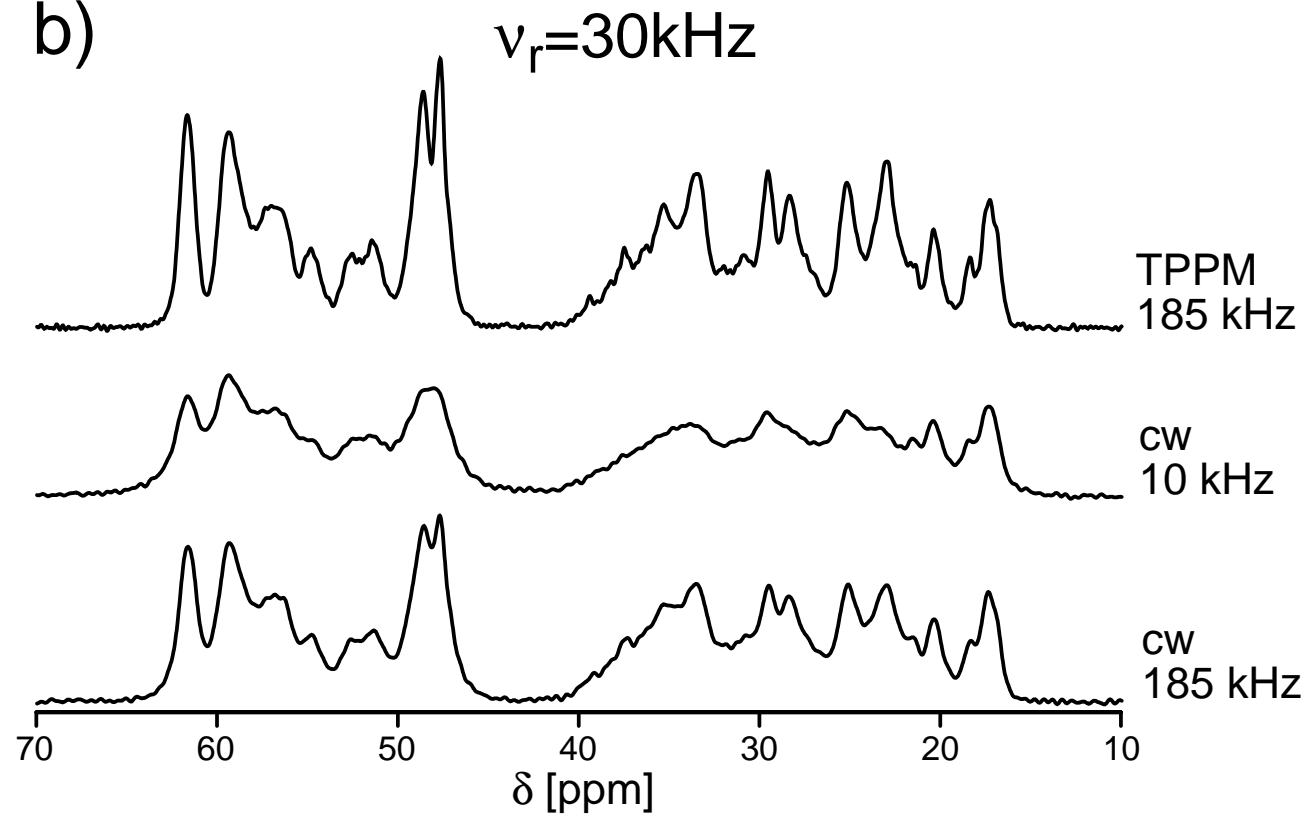

Figure 4.8: ${ }^{13} \mathrm{C}$ spectra of the cyclic decapeptide, antamanide, obtained at MAS frequencies of a) $50 \mathrm{kHz}$ and b) $30 \mathrm{kHz}$ using various decoupling schemes. 
both spectra. The same is true for the proline- $\delta$ region (ca. $46-48 \mathrm{ppm}$ ). This agrees well with the results obtained for the $\mathrm{CH}$ group in alanine (Fig. 4.5) where low-power cW decoupling reached almost the line width obtained by TPPM decoupling. In the area of the $\mathrm{CH}_{3}$-groups (ca. 17-20 ppm) there are only small differences. However, we find considerably narrower lines in the TPPM decoupled spectrum in the area of the $\mathrm{CH}_{2}$-groups (ca. 20-40 ppm), which agrees with what was in the measurements on the $\mathrm{CH}_{2}$-group in glycine (Fig. 4.7).

At $30 \mathrm{kHz}$ MAS spinning frequency (Figure 4.8b) low-power cw decoupling performs significantly worse over the whole range of chemical-shift values than either cw decoupling with an rf field strength of $185 \mathrm{kHz}$ or TPPM decoupling. This also agrees with what one would expect based on the results on alanine and glycine.

As a rule of thumb, low-power decoupling becomes an interesting alternative to high-power decoupling for spinning frequencies larger than $40 \mathrm{kHz}$. The rf-field strength should be about $1 / 4$ of the spinning frequency. At spinning frequencies larger than $50 \mathrm{kHz}$ the rf-field strength can also be set to about 2/5 of the spinning frequency (see Figure 4.4).

\subsection{References}

1. M. Ernst, A. Samoson, and B.H. Meier, Chem. Phys. Lett. 348, 293-302 (2001).

2. U. Haeberlen, High Resolution NMR in Solids: Selective Averaging. Academic Press New York, 1976.

3. M. Ernst, H. Zimmermann, and B.H. Meier, Chem. Phys. Lett. 317, 581-588 (2000).

4. M. Ernst, A Verhoeven, and B.H. Meier, J. Magn. Reson. 130, 176-185 (1998).

5. D.P. Raleigh, A.C. Kolbert, T.G. Oas, M.H. Levitt, and R.G. Griffin, J.Chem.Soc.,Faraday Trans. 84, 3691 (1988).

6. T.G. Oas, R.G. Griffin, and M.H. Levitt, J. Chem. Phys. 89, 692 (1988).

7. M.H. Levitt, T.G. Oas, and R.G. Griffin, Isr. J. Chem. 28, 271-282 (1988).

8. A.C. Kolbert, D.P. Raleigh, R.G. Griffin, and M.H. Levitt, J. Magn. Reson. 89, 133 
(1990).

9. A. Samoson, T. Tuherm, and J. Past, J. Magn. Reson. 149, 264-267 (2001).

10. N.C. Nielsen, H. Bildsoe, H.J. Jakobsen, and M.H. Levitt, J. Chem. Phys. 101, 1805 (1994).

11. I.J. Shannon, K.D.M. Harris, and S. Arumugan, Chem.Phys.Lett. 196, 588-594 (1992).

12. M. Mehring, G. Sinning, and A. Pines, Z. Phys. B 24, 73 (1976).

13. G. Sinning, M. Mehring, and A. Pines, Chem. Phys. Lett. 43, 382-386 (1976).

14. M. Mehring and G. Sinning, Phys. Rev. B: Condens. Matter 15, 2519-2532 (1977).

15. M. Ernst, S. Bush, A.C. Kolbert, and A. Pines, J. Chem. Phys. 105, 3387-3397 (1996).

16. A.J. Shaka, J. Keeler, T. Frenkiel, and R. Freeman, J. Magn. Reson. 52, 335-338 (1983).

17. A.J. Shaka, J. Keeler, and R. Freeman, J. Magn. Reson. 53, 313 (1983).

18. A. Detken, E. H. Hardy, M. Ernst, M. Kainosho, T. Kawakami, S. Aimoto, and B. H. Meier, J. Biomol. NMR 20, 203-221 (2001). 


\section{Multiple-Pulse Decoupling Under MAS}

\subsection{General Considerations}

The residual line broadening in cw-decoupled spectra is dominated by the first-order average Hamiltonian term, which consists of a cross term between the chemical-shielding tensor of the protons and the heteronuclear dipolar-coupling tensor as can be seen from Eqs. [2.11], [2.18], and [4.13] for the cases of low and high rf-field amplitudes. In principle, one can use a symmetric pulse sequence (1) to ensure that all odd orders of the average Hamiltonian are zero. A symmetric pulse sequence in this context means that in the toggling frame, the condition

$$
\tilde{\mathscr{H}}(\mathrm{t})=\tilde{\mathscr{H}}\left(\tau_{\mathrm{c}}-\mathrm{t}\right)
$$

is fulfilled. In static samples, this condition can easily be fulfilled by a simple phasealternating pulse sequence of the form $\left(360^{\circ}{ }_{+x}, 360^{\circ}{ }_{-x}\right)$. There are many more pulse sequences in liquid state NMR which are symmetric and remove the first-order average Hamiltonian (1),(2).

In rotating samples it is more complicated to fulfill this condition since one has not only the time dependence due to the toggling frame but also the time dependence due to the MAS sample rotation. One way to fulfill the condition of Eq. [5.1] is by reversing both the rotation of the sample and the phase of the rf irradiation in the center of the pulse sequence. Reversing the sample rotation is technically possible (3) but requires too much time to be practical in this context.

Introducing a multiple-pulse sequence on top of magic-angle sample spinning creates additional possibilities for recoupling conditions. Now we can have interference not only between the sample spinning and the rf-field amplitude but also with the frequencies contained in the multiple-pulse sequence. So far there are only three multiple-pulse decoupling sequences under MAS which give improved results over a large range of parameters, namely TPPM decoupling (4), $\mathrm{C} 12_{2}^{-1}$ decoupling (5), and $\mathrm{XiX}(6)$ decoupling. 


\subsection{TPPM Decoupling}

Two-pulse phase-modulated decoupling (4) consists of pulses with a flip angle $\beta_{p}$ and alternating phases $\pm \varphi / 2$. The optimum value for the flip angle is typically near $180^{\circ}$. The optimum value of the phase, $\varphi$, can vary over quite a large range and is usually between $10^{\circ}$ and $50^{\circ}$. Both the flip angle and the phase vary as a function of the spinning speed, the spectrometer, the probe, and the sample. There are some variations of the TPPM sequence (see Chapter 1.4) but none of them offers significant improvements in rigid solids (7),(8),(9),(10).

Keeping in mind the remarks about possible interference effects between a multiple-pulse sequence and the sample rotation, we can try to understand the effects of TPPM decoupling by first calculating the effects on a static sample. Using the following idealized TPPM pulse sequence $\left(180^{\circ}{ }_{+\varphi / 2}, 180^{\circ}{ }_{-\varphi / 2}\right)_{N}$ with $\varphi=\pi / \mathrm{N}$, we obtain a pulse sequence which is cyclic in the average Hamiltonian sense, and we can calculate the first two orders of the average Hamiltonian. The zeroth-order average Hamiltonian is the same as for cw decoupling (Eq. [2.17]) while the first-order average Hamiltonians for $\mathrm{N}>1$ is given by (11)

$$
\begin{aligned}
\bar{H}^{(1)}=\frac{2 \tan (\varphi / 2)}{\pi}\left[\frac{\left(\omega_{\mathrm{SI}}(\mathrm{t})+\pi \mathrm{J}_{\mathrm{SI}}\right)^{2}+\left(\omega_{\mathrm{I}}+\omega_{\mathrm{I}}(\mathrm{t})\right)^{2}}{2 \omega_{1}} \cdot \mathrm{I}_{\mathrm{x}}+\right. \\
\left.\frac{\left(\omega_{\mathrm{SI}}(\mathrm{t})+\pi \mathrm{J}_{\mathrm{SI}}\right)\left(\omega_{\mathrm{I}}+\omega_{\mathrm{I}}(\mathrm{t})\right)}{\omega_{1}} \cdot 2 S_{\mathrm{z}} \mathrm{I}_{\mathrm{x}}\right]
\end{aligned}
$$

Comparing the result of Eq. [5.2] with the equivalent result for cw decoupling (Eqs. [2.11] and [2.19]) shows that the residual second-order term obtained under the TPPM sequence is by a factor of $2 \tan (\varphi / 2) / \pi \approx \varphi / \pi$ smaller than for cw decoupling. For $\varphi$ $=40^{\circ}$ this is about 0.23 or more than a factor of four in reduction of the second-order recoupling contribution to the residual line broadening. However, one has to be careful in interpreting this result. For smaller phase angles, $\varphi$, the residual coupling term becomes smaller, but at the same time the cycle time of the sequence gets longer, necessitating consideration of the convergence of the average Hamiltonian treatment. Equation [5.2] does not reflect the experimental experience (vide infra) that one 
usually obtains distinct points in the two-dimensional parameter space where TPPM decoupling performs particularly well.

TPPM decoupling can also be explained in the context of the symmetry considerations of the so-called class of helical R sequences (12), where a simultaneous averaging over the MAS rotation and the spin rotations is considered. However, the symmetry considerations give only qualitative answers about the size of the different terms in zeroth-order and first-order average Hamiltonian theory.
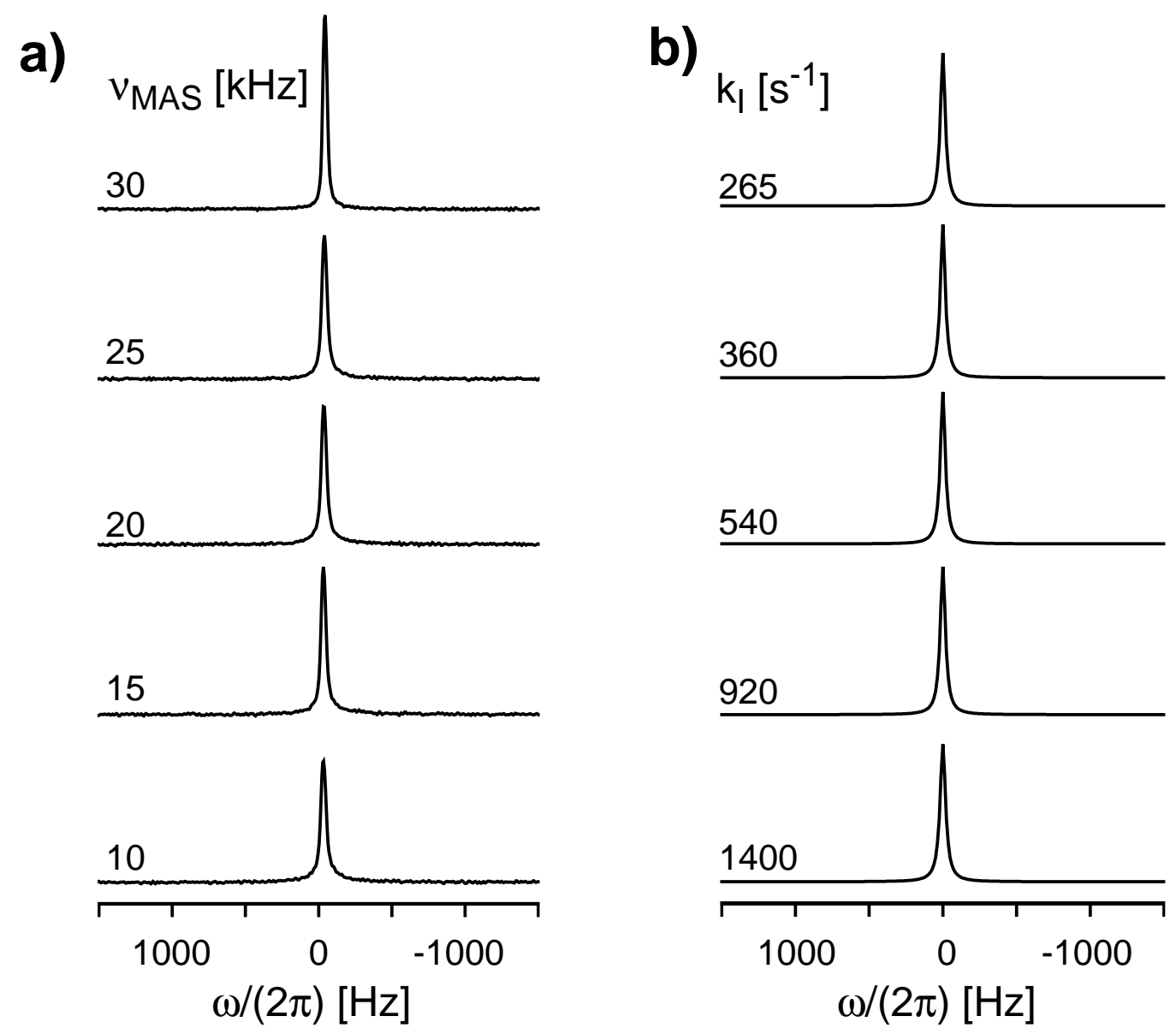

Figure 5.1: a) Measured spectra of $2-{ }^{13} \mathrm{C}$-alanine using a TPPM decoupling field strength of $\omega_{1} /(2 \pi)=100 \mathrm{kHz}$ at different spinning frequencies between $v_{\mathrm{r}}=10 \mathrm{kHz}$ and $30 \mathrm{kHz}$ at a proton resonance frequency of $600 \mathrm{MHz}$. The phase angle, $\varphi$, was set to $30^{\circ}$, and the pulse length was optimized. The simulated spectra in (b) use the measured spin-diffusion rate constants, $\mathrm{k}_{\mathrm{I}}$, under $\mathrm{cW}$ irradiation at the five spinning frequencies and assume a one-bond C$\mathrm{H}$ dipolar coupling. At all spinning frequencies, measurements and simulations result in a relatively sharp line. 
It is not clear how the proton spin-diffusion rate constant under TPPM decoupling and MAS compares to the one under cw decoupling and MAS as measured in Chapter 3.3. There is no simple way of measuring the proton spindiffusion rate constant under TPPM irradiation. It has been claimed that some of the improvements of TPPM decoupling are due to a recoupling of the proton homonuclear couplings and, therefore, an increase in the proton spin-diffusion rate constant (12). Despite this uncertainty in the size of the proton spin-diffusion rate constant, one can try to simulate TPPM decoupling in the framework of the spindiffusion model (see Chapter 3.1) assuming that the spin diffusion rate constant, $\mathrm{k}_{\mathrm{I}}$, is of the same order of magnitude as the one measured under $\mathrm{cw}$ decoupling. A comparison between measurements and simulations (see Fig. 5.1) shows quite good agreement. However, this result does not allow us to draw any conclusions about the size of the proton spin-diffusion rate constant because the simulations result in a relatively narrow line over a large range of values for the parameter $k_{I}$ (13).

The influence of the parameter $\mathrm{k}_{\mathrm{I}}$ on the performance of TPPM decoupling can be better judged from looking at full two-dimensional TPPM decoupling surfaces. Figure 5.2 shows a contour plot of the maximum line intensity for the $\mathrm{CH}$ group in 2-

a) Experiment

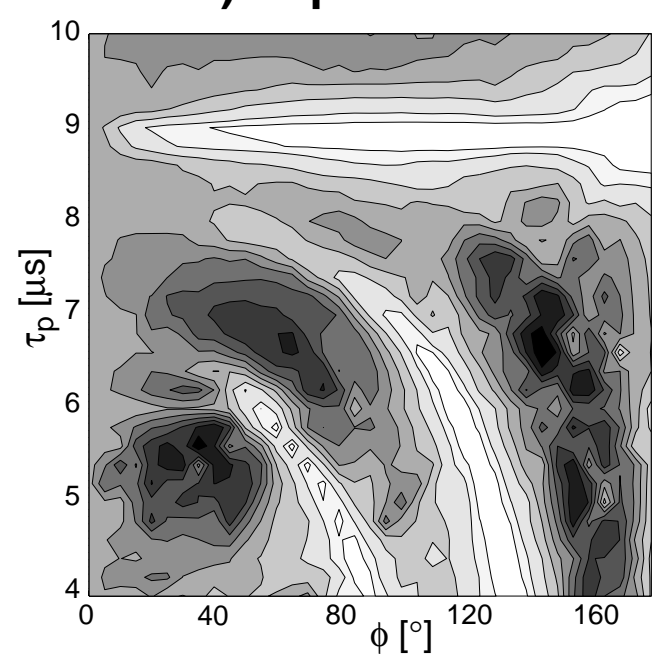

b) Simulation

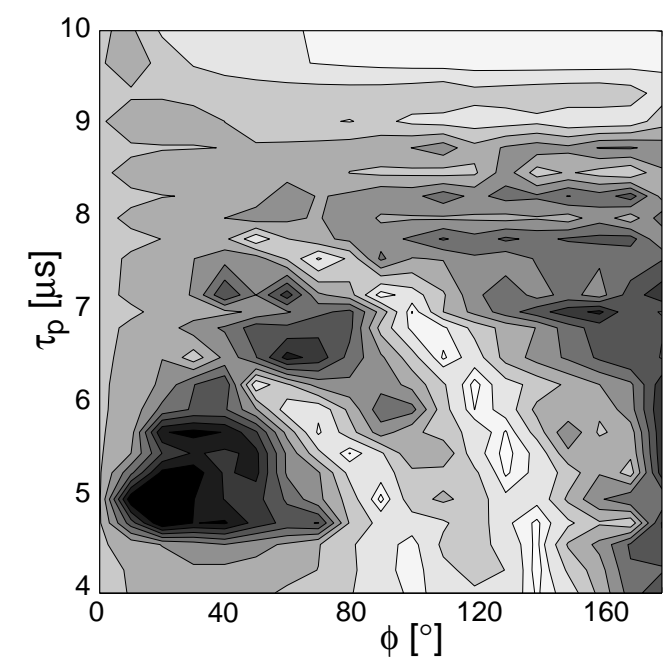

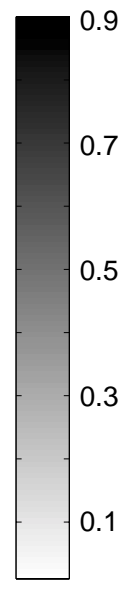

Figure 5.2: (a) Two-dimensional contour plot showing the line intensity of the $\mathrm{C}-\mathrm{H}$ group of alanine as a function of the pulse length $\left(\tau_{p}\right)$ and the phase angle $(\varphi)$ for a TPPM decoupling sequence $\tau_{-\varphi / 2} \tau_{+\varphi / 2}$. Higher intensity indicates narrower lines and therefore better decoupling. (b) Simulated contour plot assuming a proton spin-diffusion rate constant of $\mathrm{k}_{\mathrm{I}}=500 \mathrm{~s}^{-}$ 
${ }^{13} \mathrm{C}$-alanine as a function of the pulse length $\left(\tau_{p}\right)$ and the phase angle $(\varphi)$ for the TPPM decoupling sequence $\tau_{-\varphi / 2} \tau_{+\varphi / 2}$. Higher peak intensity (darker areas in Figure 5.2) indicates better decoupling. The measurements (Figure 5.2a) were done at a proton resonance frequency of $300 \mathrm{MHz}$ and at a spinning frequency of $v_{\mathrm{r}}=28 \mathrm{kHz}$. The contour plot shows a relatively broad maximum for $\tau_{\mathrm{p}} \approx 5 \mu$ s (corresponding to a $180^{\circ}$ pulse at a field strength of $\omega_{1} /(2 \pi)=100 \mathrm{kHz}$ ) and $\varphi \approx 40^{\circ}$. Considering the simplicity of the model, the general features of the experimentally measured contour plot are well represented in the simulation (Figure 5.2b), which shows that the model is able to describe the spin system in a realistic way over a large range of parameters. The simulations also reflect the various resonance conditions, which are indicated by bands of low intensity in both the simulated and the experimental data.

A similar comparison is shown in Fig. 5.3 for the N-H group in [ $\left.\mathrm{d}_{9}\right]$-trimethyl${ }^{15} \mathrm{~N}$-ammonium chloride where the rate constant $\mathrm{k}_{\mathrm{I}}=0 \mathrm{~s}^{-1}$ was assumed, i.e., a fully isolated two-spin system. Both the simulated and the measured two-dimensional intensity plots are much less smooth than in the case of the strongly coupled proton system. The areas of good decoupling are much narrower and occur for well-defined

a) Experiment

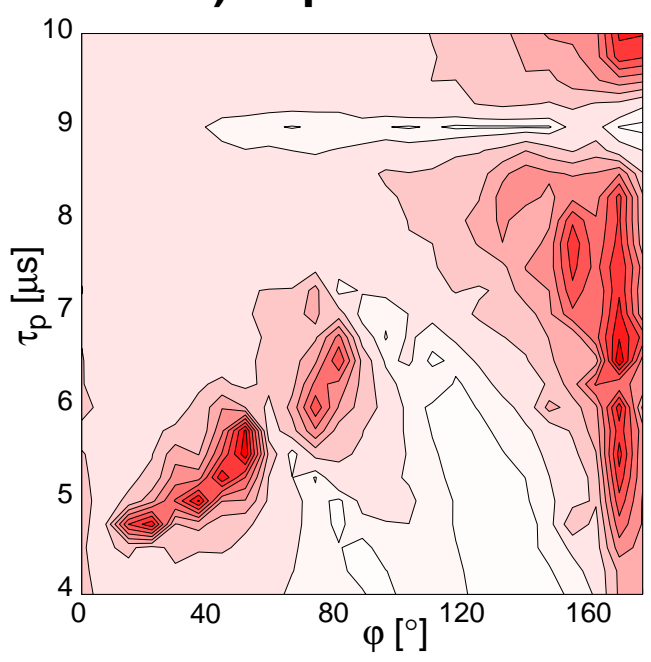

b) Simulation

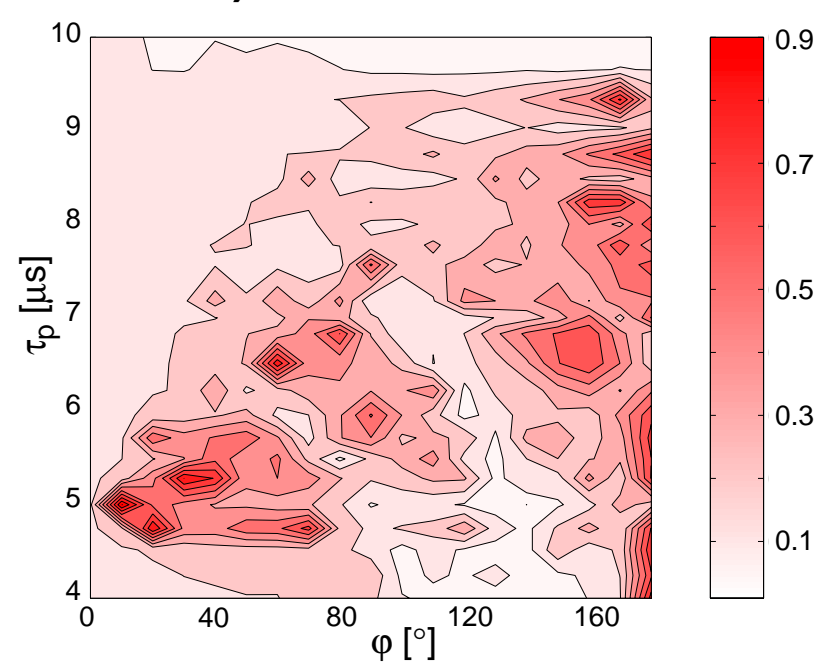

Figure 5.3: a) Two-dimensional contour plot showing the line intensity of the N-H group of $\left[\mathrm{d}_{9}\right]$-trimethyl- ${ }^{15} \mathrm{~N}$-ammonium chloride as a function of the pulse length $\left(\tau_{\mathrm{pm}}\right)$ and the phase angle $(\varphi)$ for a TPPM decoupling sequence $\tau_{-\varphi / 2} \tau_{+\varphi / 2}$. Higher intensity indicates narrower lines and therefore better decoupling. (b) Simulated contour plot assuming a proton spindiffusion rate constant of $\mathrm{k}_{\mathrm{I}}=0 \mathrm{~s}^{-1}$ and $\delta_{\mathrm{NH}} /(2 \pi)=22 \mathrm{kHz}$. 
combinations of pulse lengths and phase angles. Again, the agreement between simulation and experiment is quite good considering the simplicity of the model.

The quality of TPPM decoupling depends on two parameters: (i) the phase angle, $\varphi$, and (ii) the pulse length, $\tau_{p}$, or more precisely the flip angle, $\beta_{p}=-\omega_{1} \tau_{p}$. The optimum values for the two parameters are not easy to predict. It is, therefore, required that one optimize both parameters under the desired experimental conditions in order to obtain the best decoupling results. The dependence of the efficiency of TPPM decoupling on the flip angle, $\beta_{p}$, leads, especially at fast MAS spinning frequencies, to a high sensitivity to rf-field inhomogeneities (vide infra). This can also be seen from Fig. 5.3, which shows a very narrow optimum condition in the flip-angle dimension.

\subsection{XiX Decoupling}

The main disadvantage of TPPM decoupling is the fact that the parameters for optimum decoupling cannot be predicted easily and have to be determined experimentally each time. Such an experimental two-dimensional parameter optimization is time consuming and only possible for samples with a high signal-tonoise ratio. A decoupling sequence with only a single variable parameter would, therefore, have advantages especially if the position of the performance optimum could be predicted theoretically and would be independent of as many experimental parameters as possible. The simplest such sequence would be a phase-alternating sequence $\left(\theta_{+x}, \theta_{-x}\right)_{N}$ which has only one parameter, namely the pulse length $\tau_{p}$.

\subsubsection{Theory and Numerical Simulations}

We assume a general pulse sequence of the form $\left(\theta_{+\mathrm{x}}, \theta_{-\mathrm{x}}\right)_{\mathrm{N}}$ that spans $\mathrm{n}$ rotor cycles. The length of a single pulse is then given by $\tau_{p}=n \tau_{r} /(2 N)=\pi n /\left(N \omega_{r}\right)$, and the rf-field strength is assumed to be $\omega_{1}=p \cdot \omega_{\mathrm{r}}$. Such a sequence is cyclic in the average Hamiltonian sense (1), permitting us to calculate the zeroth-order average 
Hamiltonian for the general sequence. Assuming that the pulse length is not a multiple of a quarter of a rotor period, we obtain:

$$
\begin{aligned}
\overline{\mathscr{H}}^{(0)}= & \omega_{\mathrm{S}} \cdot \mathrm{S}_{\mathrm{z}}+\sum_{\mathrm{k}} \frac{\mathrm{N} \omega_{\mathrm{k}}}{\mathrm{np} \pi} \cdot\left(\sin \left(\frac{\mathrm{np} \pi}{\mathrm{N}}\right) \cdot \mathrm{I}_{\mathrm{kz}}+\left[\cos \left(\frac{\mathrm{np} \pi}{\mathrm{N}}\right)-1\right] \cdot \mathrm{I}_{\mathrm{ky}}\right) \\
& +\sum_{\mathrm{k}} \frac{\mathrm{N} \pi \mathrm{J}_{\mathrm{Sk}}}{\mathrm{np} \pi} \cdot\left(\sin \left(\frac{\mathrm{np} \pi}{\mathrm{N}}\right) \cdot 2 \mathrm{~S}_{\mathrm{z}} \mathrm{I}_{\mathrm{kz}}+\left[\cos \left(\frac{\mathrm{np} \pi}{\mathrm{N}}\right)-1\right] \cdot 2 \mathrm{~S}_{\mathrm{z}} \mathrm{I}_{\mathrm{ky}}\right) .
\end{aligned}
$$

This shows that to zeroth-order average Hamiltonian theory the heteronuclear dipolar coupling and the I-spin chemical-shielding tensors are fully averaged out while the isotropic heteronuclear J coupling and the isotropic chemical shift of the I spins are scaled. If the pulse length equals an integer multiple of a quarter of the rotor period, we obtain additional recoupled dipolar (and CSA) terms which have the general form

$$
\frac{\mathrm{pN}}{\mathrm{n} \pi} \cdot\left(\frac{\omega_{\mathrm{k}}^{+\mathrm{m}}+\omega_{\mathrm{k}}^{-\mathrm{m}}}{\mathrm{p}^{2}-\mathrm{m}^{2}}\right) \cdot \sin \left(\frac{\mathrm{np} \pi}{\mathrm{N}}\right) \cdot 2 \mathrm{~S}_{\mathrm{z}} \mathrm{I}_{\mathrm{kz}} .
$$

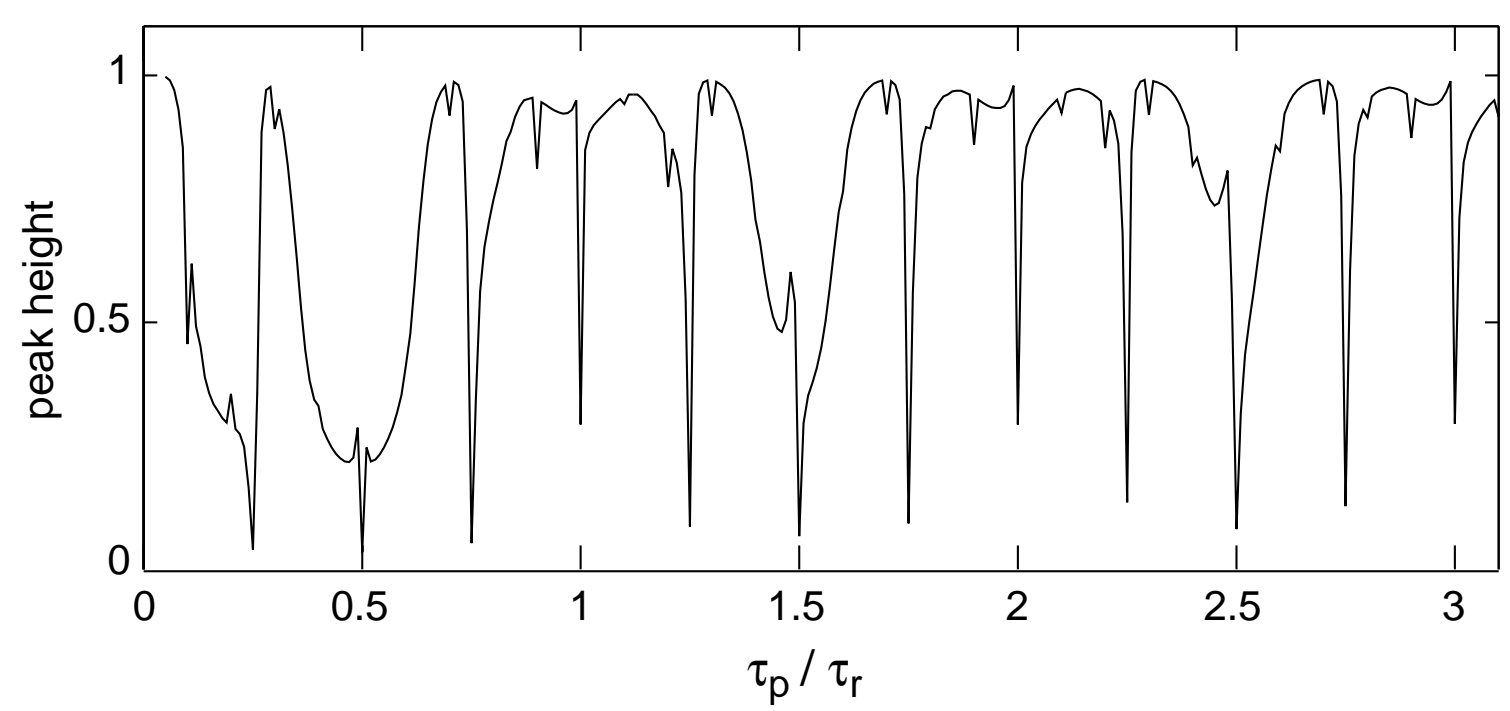

Figure 5.4: Dependence of the peak height on $\tau_{\mathrm{p}} / \tau_{\mathrm{r}}$ simulated for an isolated two-spin system. The main recoupling conditions are at the predicted locations where the pulse length is a multiple of a quarter of the rotor period. 
The precise form of the coefficients depends on the exact recoupling condition. The position of the recoupling conditions is, to zeroth order, independent of the rf-field strength.

The occurrence of the recoupling conditions at multiples of a quarter of the rotor period can also be understood by regarding the $\mathrm{XiX}$ sequence as an example of a so-called $\mathrm{CN}_{\mathrm{n}}^{v}$ sequence (5), (14), (15). For this we identify a $\mathrm{C}$ element with the basic repeat unit of the $\mathrm{XiX}$ sequence, i.e., two phase-inverted pulses of length $\tau_{\mathrm{p}}$ each. At the positions of the minima $\left(\tau_{\mathrm{p}}=\mathrm{n} \cdot \tau_{\mathrm{r}} / 4\right)$, the $\mathrm{XiX}$ sequence can then be identified with a sequence of the type $C 2_{n}^{0}$. For these sequences, symmetry considerations predict that heteronuclear dipolar interactions will be reintroduced by interference of the pulse sequence with the MAS rotation.

Numerical simulations of the S-spin intensity in an isolated two-spin system $\left(\delta_{\mathrm{SI}} /(2 \pi)=46 \mathrm{kHz}, \delta_{\mathrm{I}} /(2 \pi)=1.5 \mathrm{kHz}, v_{\mathrm{r}}=30 \mathrm{kHz}\right.$, and $\left.v_{1}=147 \mathrm{kHz}\right)$ as a function of the pulse length, $\tau_{\mathrm{p}}$, (Figure 5.4) confirm the theoretical calculations. However, they also show the limitations of lower-order average Hamiltonian calculations. The resonance conditions in the numerical simulations are quite broad, and there are many additional weaker resonances that are not predicted by the zeroth-order Hamiltonian of Eq. [5.3]. These observations reflect the fact that the zeroth-order average Hamiltonian calculation is not sufficient for a realistic description of the spin system, especially if we have to average over several rotor cycles. Calculating higherorder average Hamiltonians analytically for a general ratio $\omega_{1}=p \omega_{r}$ for $X i X$ decoupling is very time consuming.

\subsubsection{Experimental Data and Discussion}

The dependence of the experimental peak height on the pulse length in XiX decoupling is shown in Fig. 5.5a-c for two different rf-field amplitudes, $v_{1}$, and two MAS frequencies, $v_{r}$, in a sample of $2-{ }^{13} \mathrm{C}$-alanine. The peak height of 1 is calibrated with respect to experimentally optimized TPPM decoupling at the same spinning frequency and rf-field strength. The agreement between the experimental data at $30 \mathrm{kHz}$ MAS and $150 \mathrm{kHz}$ rf-field strength (Fig. 5.5a) with the numerical simulations 
in an isolated two-spin system (Fig. 5.4) is quite good. The position of the main minima agree very well, and the minima at an odd integer multiple of half a rotor cycle are considerably broader than the ones at integer multiples of a rotor cycle, which in turn are broader than those at odd integer multiples of a quarter rotor cycle. At lower rf-field amplitudes there are additional minima that are not predicted by the simulations. They are most likely due to multiple-spin effects. These weak

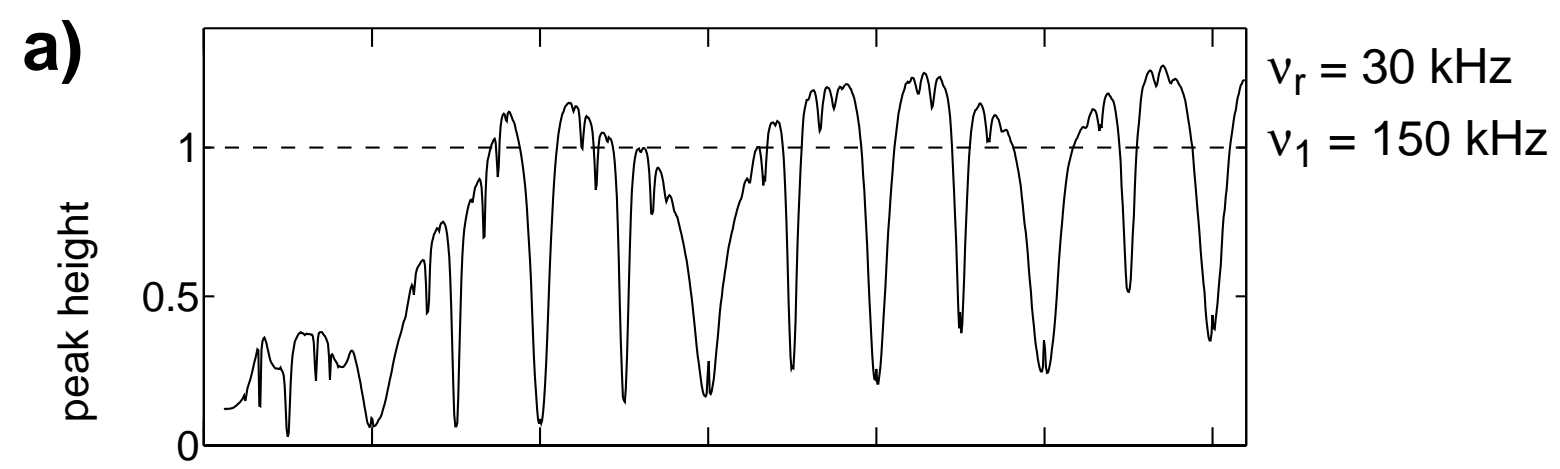

b)

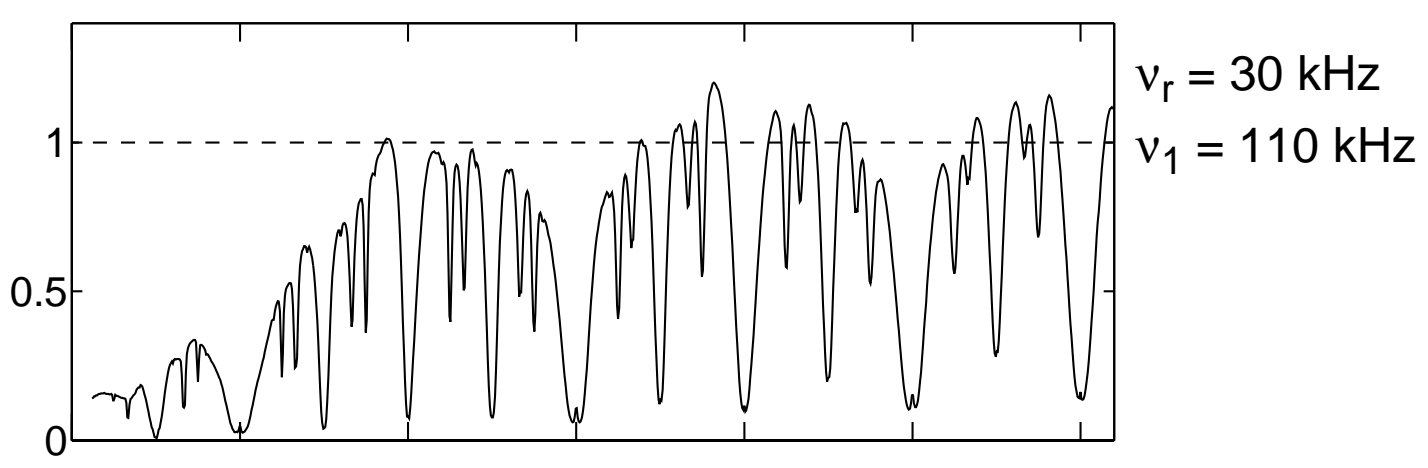

c)

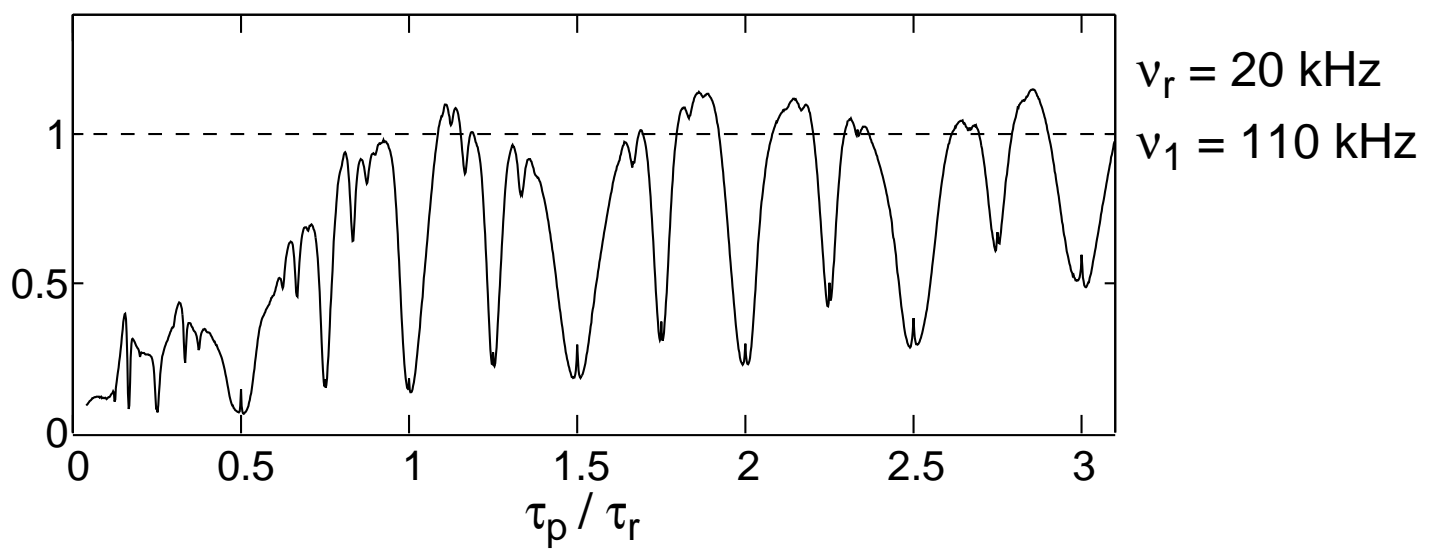

Figure 5.5: Experimental peak height under XiX decoupling as a function of the pulse length for two different spinning frequencies and two different rf-field strengths. The peak height of 1 is calibrated with respect to experimentally optimized TPPM decoupling at the same spinning frequency and rf-field strength. 
"resonances" are found whenever the pulse length approximately matches certain multiples of $1 / 24$ th of the rotor period. In the experimental data of Fig. 5.5 we find such minima for $\tau_{p}=(3 n \pm 1) \cdot \tau_{r} / 3, \tau_{p}=(6 n \pm 1) \cdot \tau_{r} / 6, \tau_{p}=(8 n \pm 1) \cdot \tau_{r} / 8$, and $\tau_{\mathrm{p}}=(8 \mathrm{n} \pm 3) \cdot \tau_{\mathrm{r}} / 8$, where $\mathrm{n}$ is an integer.

Some of these additional "resonances" get more pronounced at lower rf field amplitudes, which can be seen by comparing the measurements at $30 \mathrm{kHz}$ MAS and $150 \mathrm{kHz}$ field strength (Fig. 5.5a) with the ones at $30 \mathrm{kHz}$ MAS and $110 \mathrm{kHz}$ field strength (Fig. 5.5b). It is interesting to note that for the lower MAS frequency of $20 \mathrm{kHz}$ and an rf-field strength of $110 \mathrm{kHz}$ (Fig. 5.5c), these additional resonances are again smaller and about the same size as for the measurements in Fig. 5.5a. With increasing ratio of $\tau_{p} / \tau_{r}$, the "resonances" become weaker, but the peak height at the local performance maxima between the "resonances" decreases. Ultimately, the performance of the $\mathrm{XiX}$ sequence approaches, of course, that of $\mathrm{cw}$ decoupling. This is illustrated in Figure 5.6, which shows the same measurement as Fig. 5.5a extended to pulse lengths of up to 150 rotor periods.

In $\mathrm{XiX}$ decoupling good performance is achieved whenever the pulse length is longer than approximately one rotor period and unwanted resonance conditions, as discussed earlier, are avoided. It can be seen from Fig. 5.5, where the peak height was normalized for experimentally optimized TPPM decoupling (dashed lines), that for a broad range of pulse lengths $\tau_{p}$, the peak height under XiX decoupling is up to $20 \%$ higher than the optimized TPPM value. Even though there is a large number of undesired minima with bad decoupling performance, their positions in units of the rotor period, $\tau_{r}$, is largely independent of the rf-field amplitude and the MAS frequency. Therefore, values where a good decoupling performance is achievable can easily be predicted.

The XiX decoupling sequence is simple to set up and adjust since it depends only on a single parameter, i.e., the pulse length, $\tau_{p}$. As a good starting value, the pulse length can be adjusted to $\tau_{\mathrm{p}}=2.85 \cdot \tau_{\mathrm{r}}$ and then optimized experimentally in a relatively narrow range $\left( \pm 0.1 \cdot \tau_{r}\right)$. As the performance depends primarily only on the timing of the sequence relative to the sample rotation, transmitter instability, probe 
detuning or rf-field inhomogeneity do not critically affect the performance of the sequence.

\subsection{Comparison between TPPM and XiX Decoupling}

A more detailed comparison between the decoupling qualities of TPPM and $\mathrm{XiX}$ for different samples and under different experimental conditions is given in Fig. 5.7. Experimental spectra are shown for a $\mathrm{CH}$ group (Fig. 5.7a), using $2-{ }^{13} \mathrm{C}$-alanine as a model substance, and for a $\mathrm{CH}_{2}$ group (Fig. 5.7b), using ${ }^{2-}{ }^{13} \mathrm{C}$-glycine. The parameters of both TPPM and XiX decoupling were optimized experimentally for the two samples, the two MAS frequencies, and the three rf-field amplitudes. For each pair of spectra recorded with the two decoupling sequences, it was carefully checked

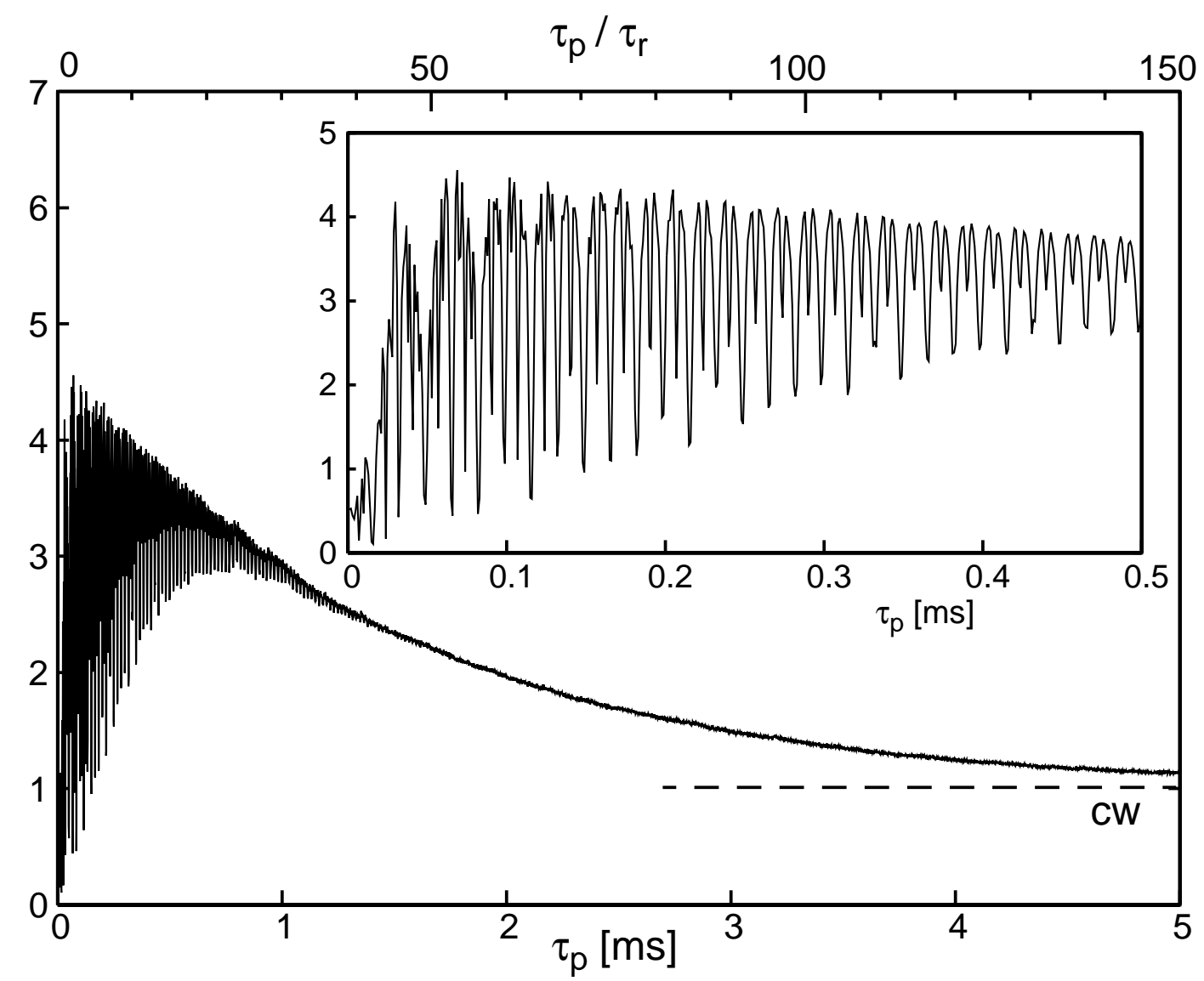

Figure 5.6: Experimental peak height under $\mathrm{XiX}$ decoupling as a function of the pulse length $\tau_{\mathrm{p}}$. For large values of $\tau_{\mathrm{p}}$ the peak height approaches the one achieved by cw decoupling. 
a)
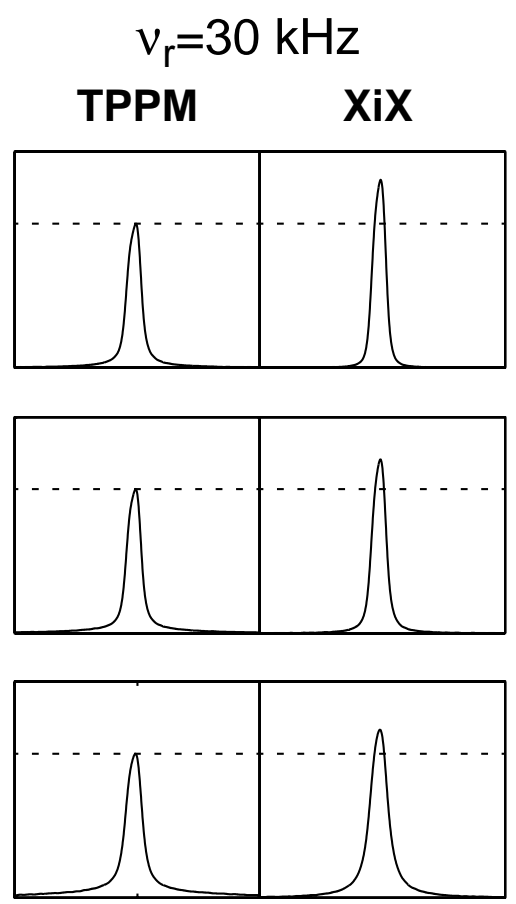

b)
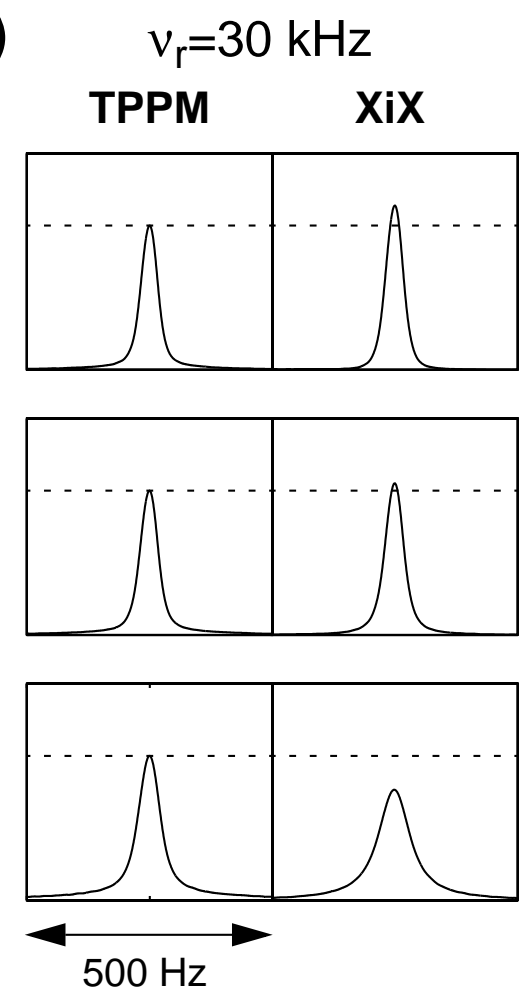

$v_{\mathrm{r}}=20 \mathrm{kHz}$

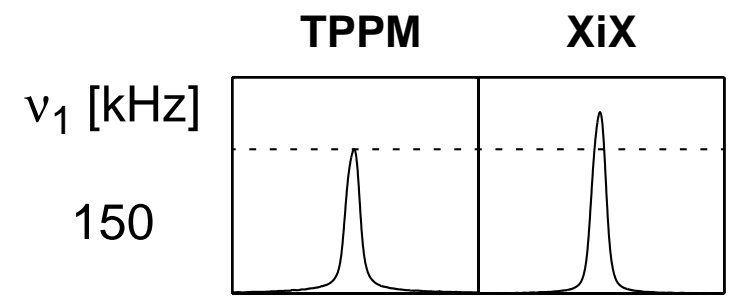

110

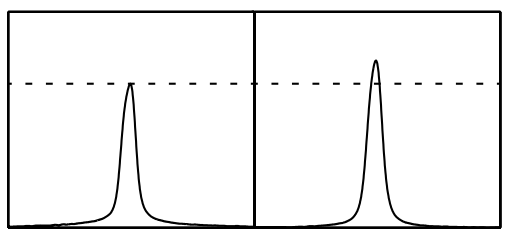

80

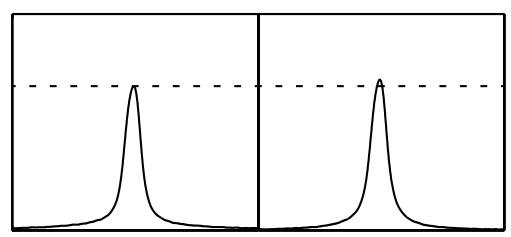

110

$$
v_{r}=20 \mathrm{kHz}
$$
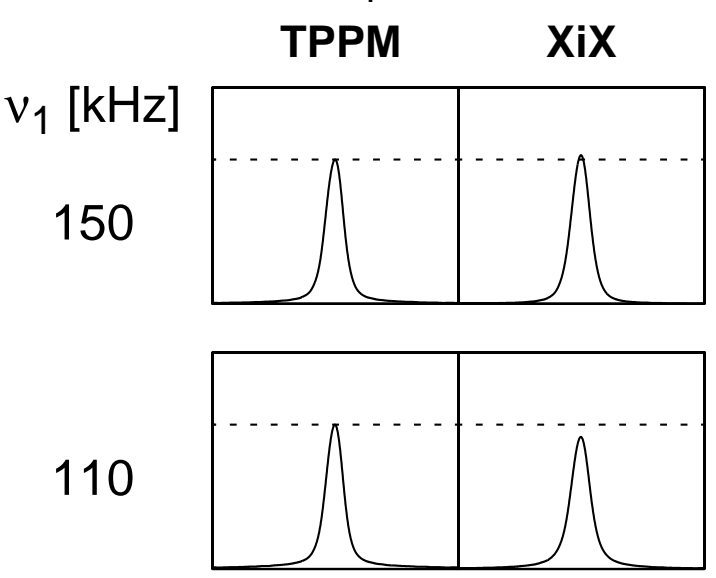

80

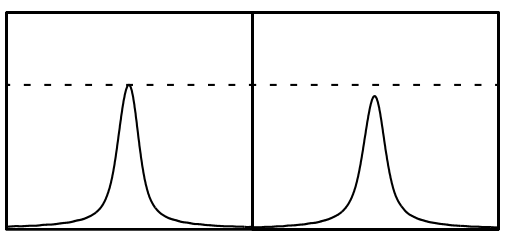

Figure 5.7: Line shape of the $C^{\alpha}$ resonances of (a) $2-{ }^{13} \mathrm{C}$-alanine and (b) $2-{ }^{13} \mathrm{C}$-glycine for TPPM and XiX decoupling at two different MAS frequencies and at three different rf-field amplitudes. For each spectrum, the parameters of the two decoupling sequences were adjusted to give maximum peak height. 
that the integrals of the resonance lines were identical within experimental uncertainty.

For the CH group (Fig. 5.7a), the height of the resonance line under optimized $\mathrm{XiX}$ decoupling is increased over the optimum height found for TPPM. As mentioned already, the line widths (FWHH) are very similar for both sequences, and the gain is primarily obtained by a sharpening of the broad 'foot' in the TPPM spectra. The largest improvement of ca. $29 \%$ in peak height is achieved at the highest rf-field amplitude of $150 \mathrm{kHz}$ and at an MAS spinning frequency of $30 \mathrm{kHz}$. The advantages of XiX over TPPM decoupling decrease with decreasing rf-field strength and decreasing MAS frequency. At a relatively low spinning frequency of $10 \mathrm{kHz}, \mathrm{XiX}$ decoupling only outperforms TPPM decoupling at an rf-field strength of $150 \mathrm{kHz}$ (data not shown) (6).

The general trends observed for the $\mathrm{CH}$ group become more pronounced for the $\mathrm{CH}_{2}$ group of glycine, as shown in Fig. 5.7b. Again, both TPPM and XiX were optimized for each spinning frequency and rf-field amplitude. While under high MAS frequencies and high rf-fields strength (e.g., $v_{\mathrm{r}}=30 \mathrm{kHz}$ and $v_{1}=150 \mathrm{kHz}$ ) gains in peak height are achieved by using XiX decoupling, TPPM decoupling offers advantages at lower rf-field strengths and slower MAS rotation.

Figure 5.8 shows the dependence of XiX (Fig. 5.8a) and TPPM (Fig. 5.8b) decoupling on the rf-field amplitude for one particular set of parameters. Only the rffield strength was changed while all other parameters were kept fixed. This corresponds to the situation that one encounters when rf-field inhomogeneities are present in a sample. These spectra were obtained from measurements on the $\mathrm{CH}$ group of labeled $2-{ }^{13} \mathrm{C}$-alanine packed in a rotor in which the sample volume was restricted to the central $1.5 \mathrm{~mm}$ along the rotor axis. The MAS frequency in all measurements was set to $30 \mathrm{kHz}$. The performance of TPPM decoupling had been optimized at a nominal rf-field amplitude of $v_{1}=130 \mathrm{kHz}$, yielding parameters $\tau_{\mathrm{p}}=$ $3.7 \mu$ s and $\varphi=35^{\circ}$. The performance of TPPM decoupling degrades significantly at lower and a higher rf-field strength. Optimization of the $\mathrm{XiX}$ sequence at $v_{1}=$ 
$130 \mathrm{kHz}$ yielded an optimum pulse length of $\tau_{\mathrm{p}}=93.7 \mu \mathrm{s}=2.81 \tau_{\mathrm{r}}$. The overall dependence on the rf-field amplitude is much weaker than for TPPM decoupling.

As mentioned above, for obtaining optimum decoupling using TPPM it is mandatory to optimize both the pulse length and the phase difference of the sequence. Predictions for the optimum values are difficult to make and, especially at
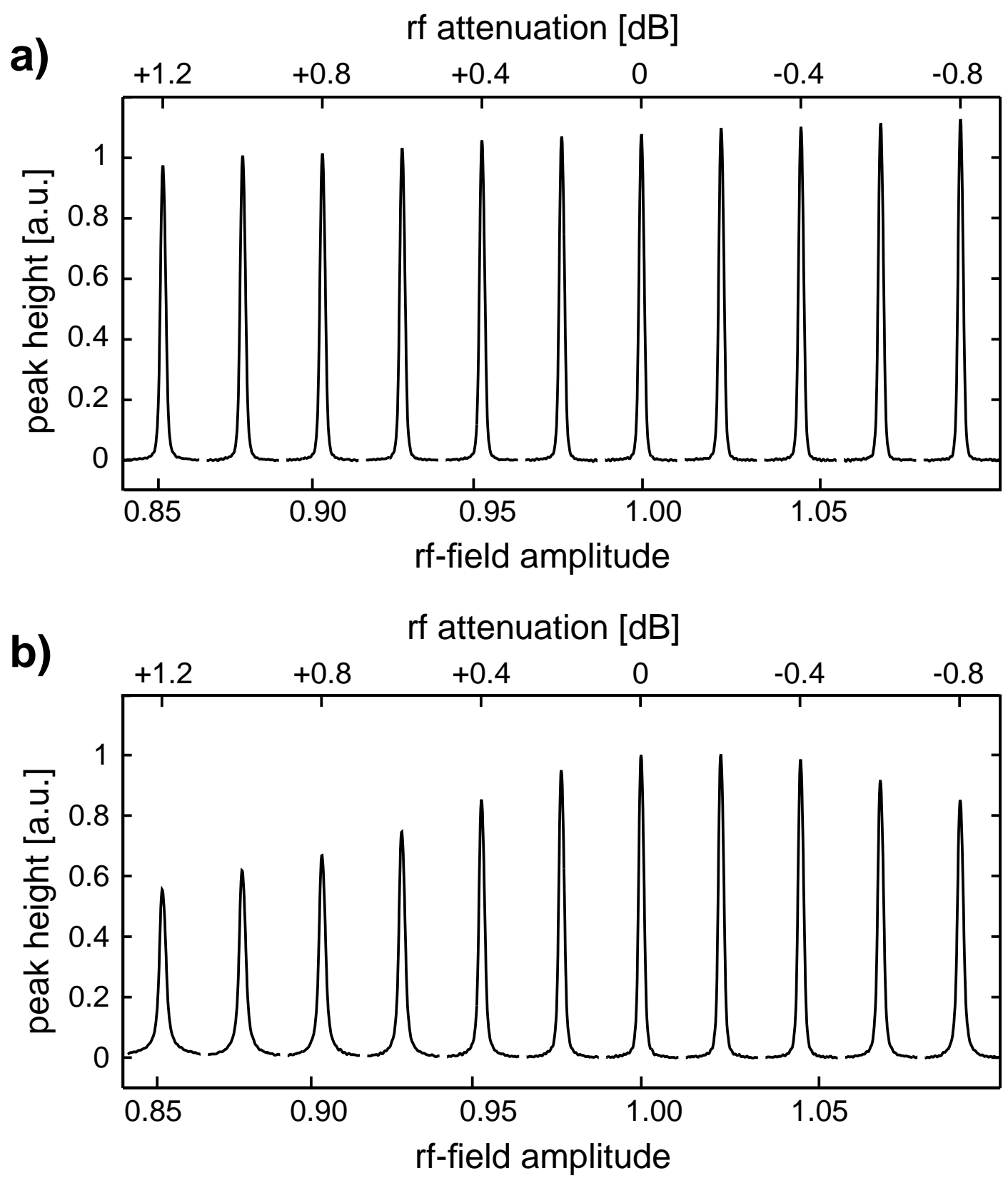

Figure 5.8: Dependence of the $\mathrm{C}^{\alpha}$ resonance of ${ }^{2-}{ }^{13} \mathrm{C}$-alanine $(\mathrm{CH}$ group) on the rf-field strength for (a) XiX and (b) TPPM decoupling. An amplitude of $v_{1}=130 \mathrm{kHz}$ corresponds to 1.00 and $0 \mathrm{~dB}$ attenuation. TPPM decoupling shows a much stronger dependence on the rffield strength than $\mathrm{XiX}$ decoupling. 
higher MAS frequencies, TPPM becomes very sensitive to the precise setting of the parameters as can be seen from Figures 5.2 and 5.3. The XiX sequence, on the other hand, only has a single parameter, and based on the MAS frequency, one can predict the locations of the performance maxima quite well. As a rule of thumb, good starting values for the pulse length when using XiX decoupling are $\tau_{\mathrm{p}}=1.85 \cdot \tau_{\mathrm{r}}$ and $\tau_{\mathrm{p}}=2.85 \cdot \tau_{\mathrm{r}}$. From these starting values a local optimization of the pulse length should be sufficient for obtaining a close-to-optimum performance.

Gains in peak height of up to $29 \%$ over TPPM can be achieved by using the XiX decoupling sequence. The gain in peak height is, in the examples shown here, mainly due to a sharpening of the broad "foot" that is observed when using TPPM or cw decoupling. In addition to a gain in resolution, application of the new sequence can lead to significant gains in sensitivity. When applied to typical organic or biological solids, the XiX sequence is best suited for high MAS frequencies (above ca. $20 \mathrm{kHz}$ ) and high rf fields (above ca. $100 \mathrm{kHz}$ ), even though gains over previously developed decoupling sequences have been observed in certain cases already at an MAS frequency of $10 \mathrm{kHz}$.

\subsection{References}

1. R.R. Ernst, G. Bodenhausen, and A. Wokaun, Principles of Nuclear Magnetic Resonance in One and Two Dimensions. Clarendon Press, Oxford, 1987.

2. U. Haeberlen, High Resolution NMR in Solids: Selective Averaging. Academic Press New York, 1976.

3. A. Samoson, Talk presented at the 2001 Gordon Research Conference on Magnetic Resonance, Bristol, Rhode Island, USA, author (2001).

4. A.E. Bennett, C.M. Rienstra, M. Auger, K.V. Lakshmi, and R.G. Griffin, J. Chem. Phys. 103, 6951-6958 (1995).

5. M. Eden and M. H. Levitt, J. Chem. Phys. 111, 1511-1519 (1999).

6. A. Detken, E. Hardy, M. Ernst, and B.H. Meier, Chem. Phys. Lett. submitted, (2002). 
7. Z.H. Gan and R.R. Ernst, Solid State NMR 8, 153-159 (1997).

8. Youlu Yu and B. M. Fung, J. Magn. Reson. 130, 317-320 (1998).

9. B.M. Fung, A.K. Khitrin, and K. Ermolaev, J. Magn. Reson. 142, 97-101 (2000).

10. K. Takegoshi, J. Mizokami, and T. Terao, Chem. Phys. Lett. 341, 540-544 (2001).

11. M. Ernst, S. Bush, A.C. Kolbert, and A. Pines, J. Chem. Phys. 105, 3387-3397 (1996).

12. M. Carravetta, M. Eden, X. Zhao, A. Brinkmann, and M. H. Levitt, Chem. Phys. Lett. 321, 205-215 (2000).

13. M. Ernst, H. Zimmermann, and B.H. Meier, Chem. Phys. Lett. 317, 581-588 (2000).

14. Y.K. Lee, N.D. Kurur, M. Helmle, O.G. Johannessen, N.C. Nielsen, and M.H. Levitt, Chem. Phys. Lett. 242, 304 (1995).

15. A. Brinkmann and M. H. Levitt, J. Chem. Phys. 115, 357-384 (2001). 


\section{Conclusions and Outlook}

The understanding of the processes which determine the residual line width under high-power cw decoupling has improved significantly over the past ten years. It is now known that the contribution from a cross term between the proton chemicalshielding tensor and the heteronuclear dipolar-coupling tensor dominates the residual linewidth. These residual coupling terms are then partially averaged out by the proton spin-diffusion process, which leads to a exchange-type process between the two transitions of the $S$ spins. Depending on the rate constant of the proton spindiffusion process, this can lead to a significant narrowing of the line compared to an isolated two-spin situation. This process has sometimes been called "self decoupling." A simple analytical model using an isotropic spin-diffusion-type superoperator allows the efficient and realistic description and simulation of $\mathrm{cw}$ decoupling in strongly-coupled spin systems. The proton spin-diffusion rate constant can be measured experimentally, allowing direct comparison of experimental results with numerical simulations.

With increasing spinning frequencies, a new method of decoupling using lowpower rf-field irradiation becomes feasible. Here, the rf-field strength has to be significantly lower than the MAS frequency while at the same time be large compared to residual coupling terms. This allows efficient decoupling at fast MAS frequencies without the need for high rf-fields that can lead to unwanted heating of the sample. The same terms dominate the residual line width in both low-power and high-power cw decoupling. It seems, therefore, not unreasonable to expect that further improvements in low-power decoupling can be achieved by using multiple-pulse sequences.

The best decoupling results are currently still achieved by high-power decoupling using multiple-pulse sequences. The progress achieved in heteronuclear spin decoupling under MAS can be seen quite well from Figure 6.1. It shows the $C^{\alpha}$ resonance of $2-{ }^{13} \mathrm{C}$-alanine under $\mathrm{cw}$ (Fig. 6.1a), optimized TPPM (Fig 6.1b), and optimized XiX (Fig. 6.1c) decoupling at the same rf-field strength of $150 \mathrm{kHz}$ and at the same MAS frequency of $30 \mathrm{kHz}$. Both TPPM and XiX decoupling show obvious 
improvements in line width and line height compared to cw decoupling. The peak height for XiX decoupling is increased by an additional 29\% over TPPM, while the line width (FWHH) is only slightly reduced, namely from $33 \mathrm{~Hz}$ (TPPM) to $31 \mathrm{~Hz}$ $(\mathrm{X} \mathrm{X})$. The reason for this apparent contradiction is that the broad 'foot' at the base of the TPPM-decoupled spectrum is reduced under $\mathrm{XiX}$ decoupling and now contributes to the central, narrow part of the line (1). Such an increase in peak height by $29 \%$ is equivalent to a decrease of the measurement time by $40 \%$ in order to obtain the same signal-to-noise ratio.

The achievable line width in a sample is, of course, not only determined by the quality of the decoupling but also by the structural homogeneity of the sample. This fact is illustrated in Figure 6.2 which shows three ${ }^{13} \mathrm{C}$ and ${ }^{15} \mathrm{~N}$ spectra recorded on a
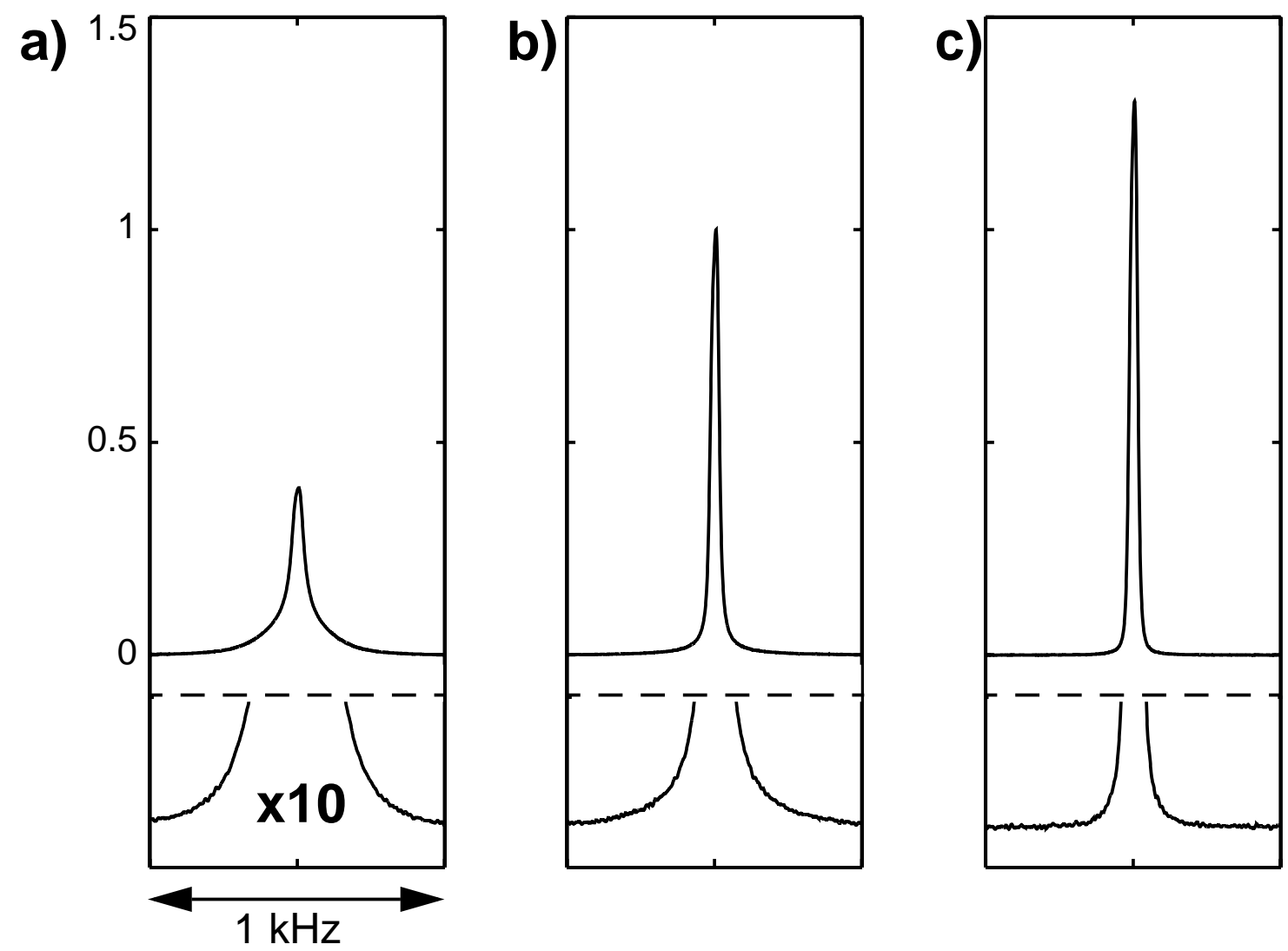

Figure 6.1: Line shape of the $C^{\alpha}$ resonance of alanine under different decoupling schemes at an rf-field amplitude of $v_{1}=150 \mathrm{kHz}$ and an MAS frequency of $30 \mathrm{kHz}$. (a) cw decoupling; (b) optimized TPPM decoupling $\left(\tau_{\mathrm{p}}=3.1 \mu \mathrm{s}\right.$ and $\left.\varphi=27^{\circ}\right)$; (c) optimized XiX decoupling $\left(\tau_{\mathrm{p}}=\right.$ $94.9 \mu \mathrm{s})$. 
sample of antamanide under different sample-preparation techniques (2). The spectra of the lyophilized sample (Figure 6.2a) show very broad lines and improving the decoupling quality will have no visible influence on the one-dimensional spectra. The spectra of the slowly crystallized sample (Figure 6.2c) show narrow and well-resolved lines and improvements in the decoupling quality might reduce the line width even further. This example clearly illustrates that heteronuclear decoupling is only one of several aspect which are important for obtaining well resolved spectra.

However, even for samples with a large heterogeneous broadening like the ones shown in Figure 6.2a, reducing the homogeneous linewidth can be important for certain experiments. In the solid-state implementation of the INADEQUATE-CR experiment (3) only the homogeneous part of the line width determines the sensitivity of the experiments, while the heterogeneous part of the line width is not important.

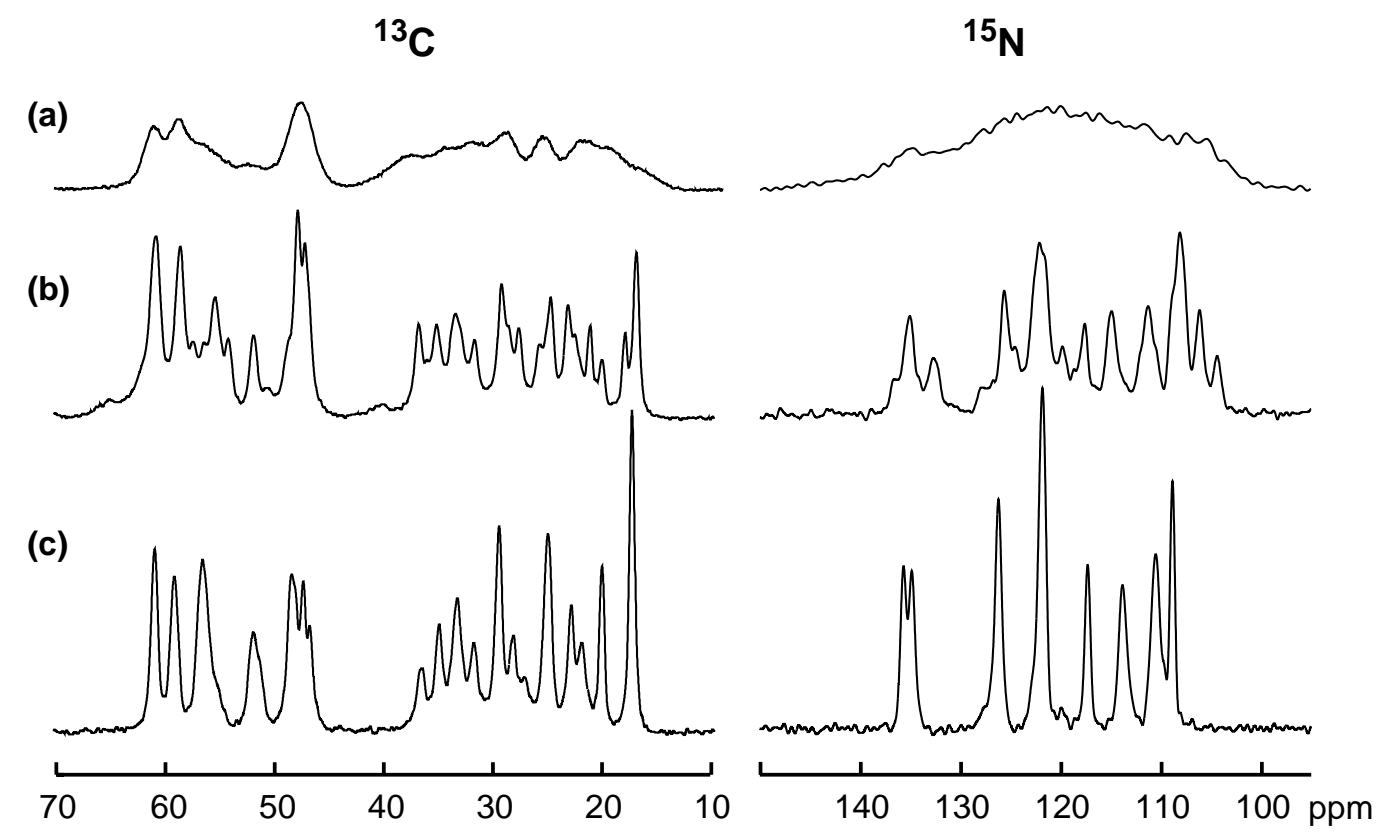

Figure 6.2: Selection of ${ }^{13} \mathrm{C}$ and ${ }^{15} \mathrm{~N}$ MAS spectra of antamanide to illustrate the influence of sample preparation on the achievable line width. (a) Lyophilized powder. (b, c) Microcrystalline powders obtained by evaporation of the solvent from a solution of antamanide in a 7:3 methanol/water mixture. The sample leading to spectra (b) was obtained by fast evaporation of the solvent at room temperature in the presence of dry silica gel as a drying agent. The sample leading to spectra (c) was obtained by slow evaporation during several days in a controlled humidity of $76 \%$. 
Therefore, in this experiment it is important to use the best possible decoupling even in the presence of heterogeneous broadening due to structural inhomogeneities in the sample. In the context of two-dimensional correlation spectroscopy the distinction between the heterogeneous and the homogeneous line width is also important since they will lead to differences in the line shape.

It is not clear whether the line width achievable under $\mathrm{XiX}_{\mathrm{X}}$ decoupling is still dominated by residual coupling terms or by the isotropic chemical-shift distribution of the samples. The first contribution to the line width could be reduced by further improvements in the decoupling sequences, while the latter would not be influenced. Most likely both contributions to the line width are still present and their relative contributions are sample dependent.

\subsection{References}

1. A. Detken, E. Hardy, M. Ernst, and B.H. Meier, Chem. Phys. Lett. submitted, (2002).

2. A. Detken, E. H. Hardy, M. Ernst, M. Kainosho, T. Kawakami, S. Aimoto, and B. H. Meier, J. Biomol. NMR 20, 203-221 (2001).

3. R. Verel, J.D. van Beek, and B.H Meier, J. Magn. Reson. 140, 300-303 (1999). 


\section{$7 \quad$ Appendices}

\subsection{Appendix A}

The explicit expressions for all the Hamiltonians under magic-angle spinning used in Eq. [1.1] are given here. They can also found in the literature (1), (2), (3) but the notation might be different. The index $\mathrm{k}$ for the I spins was replaced by an index I in all equations where only a heteronuclear two-spin system was considered.

Isotropic chemical shifts of spins S and I:

$$
\begin{gathered}
\mathscr{H}_{\mathrm{S}}^{\mathrm{CS}}=\omega_{\mathrm{S}}^{\text {iso }} \cdot \mathrm{S}_{\mathrm{z}} \\
\mathscr{H}_{\mathrm{I}}^{\mathrm{CS}}=\sum_{\mathrm{k}} \omega_{\mathrm{k}}^{\text {iso }} \cdot \mathrm{I}_{\mathrm{kz}}
\end{gathered}
$$

where $\omega_{S}^{\text {iso }}$ and $\omega_{k}^{\text {iso }}$ are the isotropic chemical shifts of spins $S$ and $I_{k}$, respectively. Homonuclear isotropic J coupling:

$$
\mathscr{H}_{\mathrm{II}}^{\mathrm{J}}=\sum_{\mathrm{k}<\ell} \pi \mathrm{J}_{\mathrm{k} \ell} \cdot 2 \overrightarrow{\mathrm{I}}_{\mathrm{k}} \overrightarrow{\mathrm{I}}_{\ell}
$$

where $\mathrm{J}_{\mathrm{k} \ell}$ is the isotropic J-coupling constant between spins $\mathrm{I}_{\mathrm{k}}$ and $\mathrm{I}_{\ell}$.

Heteronuclear isotropic J coupling:

$$
\mathscr{H}_{\mathrm{SI}}^{\mathrm{J}}=\sum_{\mathrm{k}} \pi \mathrm{J}_{\mathrm{Sk}} \cdot \mathrm{S}_{\mathrm{z}} \mathrm{I}_{\mathrm{kz}}
$$

where $\mathrm{J}_{\mathrm{Sk}}$ is the isotropic J-coupling constant between spins $\mathrm{S}$ and $\mathrm{I}_{\mathrm{k}}$.

Anisotropic chemical-shielding tensors of spins S and I:

$$
\begin{gathered}
\mathscr{H}_{\mathrm{S}}^{\mathrm{CSA}}(\mathrm{t})=\omega_{\mathrm{S}}(\mathrm{t}) \cdot \mathrm{S}_{\mathrm{z}}=\sum_{\substack{\mathrm{m}=-2 \\
\mathrm{~m} \neq 0}}^{2} \omega_{\mathrm{S}}^{+\mathrm{m}} \cdot \mathrm{e}^{-\mathrm{im} \omega_{\mathrm{r}} \mathrm{t}} \cdot \mathrm{S}_{\mathrm{z}} \\
\mathcal{H}_{\mathrm{I}}^{\mathrm{CSA}}(\mathrm{t})=\sum_{\mathrm{k}} \omega_{\mathrm{k}}(\mathrm{t}) \cdot \mathrm{I}_{\mathrm{kz}}=\sum_{\substack{\mathrm{m} \\
\mathrm{m} \neq 0}}^{2} \sum_{\mathrm{k}}^{2} \omega_{\mathrm{k}}^{+\mathrm{m}} \cdot \mathrm{e}^{-\mathrm{im} \omega_{\mathrm{r}} \mathrm{t}} \cdot \mathrm{I}_{\mathrm{kz}}
\end{gathered}
$$


where $\omega_{S}(t)$ and $\omega_{k}(t)$ are the time-dependent chemical-shielding tensors of spins $S$ and $I_{k}$, respectively. The constants $\omega_{S}^{m}$ and $\omega_{k}^{m}$ are the corresponding Fourier coefficients defined below.

Homonuclear dipolar coupling tensor of spins I:

$$
\begin{aligned}
\mathcal{H}_{\mathrm{II}}^{\mathrm{dip}}(\mathrm{t}) & =\sum_{\mathrm{k}<\ell} \omega_{\mathrm{k} \ell}(\mathrm{t}) \cdot\left[2 \mathrm{I}_{\mathrm{kz}} \mathrm{I}_{\ell \mathrm{z}}-\left(\mathrm{I}_{\mathrm{kx}} \mathrm{I}_{\ell \mathrm{x}}+\mathrm{I}_{\mathrm{ky}} \mathrm{I}_{\ell \mathrm{y}}\right)\right] \\
& =\sum_{\substack{\mathrm{k}<\ell_{\mathrm{m}}=-2 \\
\mathrm{~m} \neq 0}}^{2} \omega_{\mathrm{k} \ell}^{+\mathrm{m}} \cdot \mathrm{e}^{-\mathrm{im} \omega_{\mathrm{r}} \mathrm{t}} \cdot\left[2 \mathrm{I}_{\mathrm{kz}} \mathrm{I}_{\ell \mathrm{z}}-\left(\mathrm{I}_{\mathrm{kx}} \mathrm{I}_{\ell \mathrm{x}}+\mathrm{I}_{\mathrm{ky}} \mathrm{I}_{\ell \mathrm{y}}\right)\right]
\end{aligned}
$$

where $\omega_{\mathrm{k} \ell}(\mathrm{t})$ are the time-dependent, dipolar-coupling tensors between spins $\mathrm{I}_{\mathrm{k}}$ and $\mathrm{I}_{\ell}$ with the Fourier coefficients $\omega_{\mathrm{k} \ell}^{\mathrm{m}}$.

Heteronuclear dipolar coupling tensor between spins $S$ and I:

$$
\mathcal{H}_{\mathrm{SI}}^{\mathrm{dip}}(\mathrm{t})=\sum_{\mathrm{k}} \omega_{\mathrm{Sk}}(\mathrm{t}) \cdot 2 \mathrm{~S}_{\mathrm{z}} \mathrm{I}_{\mathrm{kz}}=\sum_{\substack{\mathrm{k} \\ \mathrm{m}=-2 \\ \mathrm{~m} \neq 0}}^{2} \omega_{\mathrm{Sk}}^{+\mathrm{m}} \cdot \mathrm{e}^{-\mathrm{im} \omega_{\mathrm{r}} \mathrm{t}} \cdot 2 \mathrm{~S}_{\mathrm{z}} \mathrm{I}_{\mathrm{kz}}
$$

where $\omega_{\mathrm{Sk}}(\mathrm{t})$ are the time-dependent dipolar-coupling tensors between spins $\mathrm{S}$ and $\mathrm{I}_{\mathrm{k}}$ with the Fourier coefficients $\omega_{\mathrm{Sk}}^{\mathrm{m}}$.

Rf irradiation on the I spins:

$$
\mathscr{H}_{\mathrm{I}}^{\mathrm{rf}}(\mathrm{t})=\omega_{1}(\mathrm{t}) \cdot \sum_{\mathrm{k}} \mathrm{I}_{\mathrm{kx}}
$$

where $\omega_{1}(t)$ is the (possibly) time-dependent rf-field amplitude. This time dependence is not due to the MAS rotation but due to the rf-irradiation scheme.

The Fourier coefficients of Eqs. [7.5] and [7.6] are defined as

$$
\begin{aligned}
& \omega_{\mathrm{S}}^{\mathrm{m}}=\frac{2}{\sqrt{6}} \cdot \mathrm{d}_{\mathrm{m}, 0}^{2}\left(\theta_{\mathrm{r}}\right) \cdot \mathrm{e}^{-\mathrm{im} \gamma_{\mathrm{s}}} \cdot \sum_{\mathrm{n}=-2}^{2} \mathrm{~d}_{\mathrm{n}, \mathrm{m}}^{2}\left(\beta_{\mathrm{S}}\right) \cdot \mathrm{e}^{-\mathrm{in} \alpha_{\mathrm{s}}} \cdot \rho_{2, \mathrm{n}}^{(\mathrm{S})} \\
& \omega_{\mathrm{k}}^{\mathrm{m}}=\frac{2}{\sqrt{6}} \cdot \mathrm{d}_{\mathrm{m}, 0}^{2}\left(\theta_{\mathrm{m}}\right) \cdot \mathrm{e}^{-\mathrm{im} \gamma_{\mathrm{k}}} \cdot \sum_{\mathrm{n}=-2}^{2} \mathrm{~d}_{\mathrm{n}, \mathrm{m}}^{2}\left(\beta_{\mathrm{k}}\right) \cdot \mathrm{e}^{-\mathrm{in} \alpha_{\mathrm{k}}} \cdot \rho_{2, \mathrm{n}}^{(\mathrm{k})}
\end{aligned}
$$


where $d_{m, n}^{2}(\varphi)$ is the reduced Wigner matrix element; $\theta_{r}$ is the rotation angle; $\alpha, \beta$, and $\gamma$ are the three Euler angles which describe the orientation of the chemicalshielding tensor in the rotor-fixed frame; and $\rho_{2, n}$ are the elements of the chemicalshielding tensor in spherical-tensor notation. They are defined as:

$$
\begin{aligned}
\rho_{2,0} & =\sqrt{\frac{3}{2}} \cdot \delta^{\mathrm{CSA}} \\
\rho_{2, \pm 1} & =0 \\
\rho_{2, \pm 2} & =\frac{1}{2} \cdot \delta^{\mathrm{CSA}} \cdot \eta^{\mathrm{CSA}}
\end{aligned}
$$

Here, the anisotropy of the chemical-shielding tensor, $\delta^{\mathrm{CSA}}$, is defined in angular frequency units and $\eta^{\text {CSA }}$ is the asymmetry of the chemical-shielding tensor.

The Fourier coefficients of the dipolar-coupling tensors of Eqs. [7.7] and [7.8] are defined as:

$$
\begin{aligned}
\omega_{\mathrm{k} \ell}^{\mathrm{m}} & =\frac{1}{\sqrt{6}} \cdot \mathrm{d}_{\mathrm{m}, 0}^{2}\left(\theta_{\mathrm{r}}\right) \cdot \mathrm{e}^{-\mathrm{im} \gamma_{\mathrm{k} \ell}} \cdot \mathrm{d}_{0, \mathrm{~m}}^{2}\left(\beta_{\mathrm{k} \ell}\right) \cdot \rho_{2,0}^{(\mathrm{k} \ell)} \\
& =\frac{1}{2 \sqrt{3|\mathrm{~m}|}}\left|\mathrm{d}_{0 . \mathrm{m}}^{2}\left(\beta_{\mathrm{k} \ell}\right)\right| \cdot \mathrm{e}^{-\mathrm{im} \gamma_{\mathrm{k} \ell}} \cdot \delta_{\mathrm{k} \ell}^{\mathrm{dip}} \quad\left(\text { for } \theta_{\mathrm{r}}=\theta_{\mathrm{m}} \approx 54.74^{\circ}\right) \\
\omega_{\mathrm{Sk}}^{\mathrm{m}} & =\frac{1}{\sqrt{6}} \cdot \mathrm{d}_{\mathrm{m}, 0}^{2}\left(\theta_{\mathrm{r}}\right) \cdot \mathrm{e}^{-\mathrm{im} \gamma_{\mathrm{sk}}} \cdot \mathrm{d}_{0, \mathrm{~m}}^{2}\left(\beta_{\mathrm{Sk}}\right) \cdot \rho_{2,0}^{(\mathrm{Sk})} \\
& =\frac{1}{2 \sqrt{3|\mathrm{~m}|}}\left|\mathrm{d}_{0 . \mathrm{m}}^{2}\left(\beta_{\mathrm{Sk}}\right)\right| \cdot \mathrm{e}^{-\mathrm{im} \gamma_{\mathrm{Sk}}} \cdot \delta_{\mathrm{Sk}}^{\mathrm{dip}} \quad\left(\text { for } \theta_{\mathrm{r}}=\theta_{\mathrm{m}} \approx 54.74^{\circ}\right)
\end{aligned}
$$

where $d_{m, n}^{2}(\varphi)$ is the reduced Wigner matrix element; and the angles $\beta$ and $\gamma$ are the two Euler angles describing the orientation of the dipolar-coupling tensor in the rotorfixed frame. The spherical-tensor elements are given by

$$
\rho_{2,0}^{(\mathrm{k} \ell)}=\sqrt{\frac{3}{2}} \cdot \delta_{\mathrm{k} \ell}^{\mathrm{dip}}
$$

and for $m \neq 0$

$$
\rho_{2, \mathrm{~m}}^{(\mathrm{k} \ell)}=0
$$

Here, 


$$
\delta_{\mathrm{k} \ell}^{\mathrm{dip}}=-2 \cdot \frac{\mu_{0}}{4 \pi} \cdot \frac{\gamma_{\mathrm{k}} \gamma_{\ell} \hbar}{\mathrm{r}_{\mathrm{k} \ell}^{3}}
$$

is the anisotropy of the dipolar-coupling tensor in units of angular frequency. The second line of Eqs. [7.13] and [7.14] is only valid for $\theta_{\mathrm{r}}=\theta_{\mathrm{m}} \approx 54.74^{\circ}$ (magic angle). For the heteronuclear dipolar-coupling tensor, analogous expressions hold true.

\subsection{Appendix B}

The six coefficients in Eqs. [4.5]-[4.8] can be calculated from the Fourier coefficients of the various time-dependent Hamiltonians given in Eqs. [7.10]-[7.14]. All calculations were carried out using the Mathematica program by Wolfram Research.

The coefficient for the cross term between the heteronuclear coupling (anisotropic dipolar coupling plus isotropic J coupling) and the homonuclear coupling (anisotropic dipolar coupling plus isotropic J coupling) is given by:

$$
\bar{\omega}_{\mathrm{Sk} \ell}=\sum_{\substack{\mathrm{m}=-2 \\ \mathrm{~m} \neq 0}}^{2} \frac{\mathrm{i}}{2 \mathrm{~m}} \cdot\left[\left(\omega_{\mathrm{k} \ell}^{+\mathrm{m}}-2 \pi \mathrm{J}_{\mathrm{k} \ell}\right) \cdot\left(\omega_{\mathrm{Sk}}^{-\mathrm{m}}-\omega_{\mathrm{S} \ell}^{-\mathrm{m}}\right)-\left(\pi \mathrm{J}_{\mathrm{Sk}}-\pi \mathrm{J}_{\mathrm{S} \ell}\right) \cdot \omega_{\mathrm{k} \ell}^{+\mathrm{m}}\right] \text {. }
$$

The coefficient for the cross term between the homonuclear coupling (anisotropic dipolar coupling plus isotropic J coupling) and the chemical shift of spin I (anisotropic chemical-shielding tensor plus isotropic chemical shift) is given by:

$$
\bar{\omega}_{\mathrm{k} \ell}=\sum_{\substack{\mathrm{m}=-2 \\ \mathrm{~m} \neq 0}}^{2} \frac{\mathrm{i}}{2 \mathrm{~m}} \cdot\left[\left(\omega_{\mathrm{k} \ell}^{+\mathrm{m}}-2 \pi \mathrm{J}_{\mathrm{k} \ell}\right) \cdot\left(\omega_{\mathrm{k}}^{-\mathrm{m}}-\omega_{\ell}^{-\mathrm{m}}\right)-\left(\omega_{\mathrm{k}}-\omega_{\ell}\right) \cdot \omega_{\mathrm{k} \ell}^{+\mathrm{m}}\right] .
$$

The coefficient for the first-order term of the homonuclear coupling (anisotropic dipolar coupling plus isotropic J coupling) with itself is given by: 


$$
\begin{aligned}
\bar{\omega}_{\mathrm{nk} \ell}=\sum_{\mathrm{m}=-2}^{2} \frac{\mathrm{i}}{4 \mathrm{~m}} \cdot\left[\omega_{\mathrm{nk}}^{-\mathrm{m}} \omega_{\mathrm{n} \ell}^{+\mathrm{m}}+2 \omega_{\mathrm{n} \ell}^{+\mathrm{m}} \omega_{\mathrm{k} \ell}^{-\mathrm{m}}+2 \omega_{\mathrm{nk}}^{-\mathrm{m}} \omega_{\mathrm{k} \ell}^{+\mathrm{m}}\right. \\
+\omega_{\mathrm{nk}}^{-\mathrm{m}}\left(2 \pi \mathrm{J}_{\mathrm{n} \ell}+4 \pi \mathrm{J}_{\mathrm{k} \ell}\right) \\
+\omega_{\mathrm{n} \ell}^{+\mathrm{m}}\left(2 \pi \mathrm{J}_{\mathrm{nk}}+4 \pi \mathrm{J}_{\mathrm{k} \ell}\right) \\
\left.+\omega_{\mathrm{k} \ell}^{-\mathrm{m}}\left(2 \pi \mathrm{J}_{\mathrm{nk}}-2 \pi \mathrm{J}_{\mathrm{n} \ell}\right)\right]
\end{aligned}
$$

The coefficient for the cross terms between the rf field and the anisotropic chemicalshielding tensor is given by:

$$
\bar{\omega}_{\mathrm{k}}^{\mathrm{rf}}=\sum_{\substack{\mathrm{m}=-2 \\ \mathrm{~m} \neq 0}}^{2} \frac{\mathrm{i}}{\mathrm{m}} \cdot \omega_{\mathrm{k}}^{+\mathrm{m}}
$$

The coefficients for the cross term between the rf field and the anisotropic heteronuclear dipolar-coupling tensor is given by:

$$
\bar{\omega}_{\mathrm{Sk}}^{\mathrm{rf}}=\sum_{\substack{\mathrm{m}=-2 \\ \mathrm{~m} \neq 0}}^{2} \frac{\mathrm{i}}{\mathrm{m}} \cdot \omega_{\mathrm{Sk}}^{+\mathrm{m}}
$$

The coefficients for the cross term between the rf field and the anisotropic homonuclear dipolar-coupling tensor is given by:

$$
\bar{\omega}_{\mathrm{k} \ell}^{\mathrm{rf}}=\sum_{\substack{\mathrm{m}=-2 \\ \mathrm{~m} \neq 0}}^{2} \frac{3 \mathrm{i}}{2 \mathrm{~m}} \cdot \omega_{\mathrm{k} \ell}^{+\mathrm{m}} .
$$

The coefficient of [4.10] is given by:

$$
\bar{\omega}_{\mathrm{nk} \ell}^{\prime}=\sum_{\substack{\mathrm{m}=-2 \\ \mathrm{~m} \neq 0}}^{2} \frac{\mathrm{i}}{4 \mathrm{~m}} \cdot\left[4 \omega_{\mathrm{nk}}^{+\mathrm{m}} \omega_{\mathrm{n} \ell}^{-\mathrm{m}}+\omega_{\mathrm{n} \ell}^{+\mathrm{m}} \omega_{\mathrm{k} \ell}^{-\mathrm{m}}-\omega_{\mathrm{nk}}^{+\mathrm{m}} \omega_{\mathrm{k} \ell}^{-\mathrm{m}}\right]
$$

For simplicity, only the homonuclear dipolar coupling has been considered in the above expression while the homonuclear J coupling has been omitted. 


\subsection{References}

1. M. Mehring, Principles of High Resolution NMR in Solids, 2nd edition. Springer, Berlin, 1983.

2. U. Haeberlen, High Resolution NMR in Solids: Selective Averaging. Academic Press New York, 1976.

3. K.Schmidt-Rohr and H.W. Spiess, Multidimensional Solid-State NMR and Polymers. Academic Press, London, 1994. 


\section{Acknowledgements}

I would like to thank Prof. Alexander Pines, University of California at Berkeley, who started my interest in heteronuclear spin decoupling by asking me to work on second-order effects in heteronuclear spin systems. During my time in Berkeley I worked closely with Dr. Andrew Kolbert and Dr. Seth Bush on trying to understand how cw decoupling works under MAS.

Most of the work described in this "Habilitationsschrift" was carried out in the group of Prof. Beat H. Meier first at the University of Nijmegen and now at the ETH Zürich. I would like to thank him for the friendship, freedom, independence, support, and many stimulating discussions and suggestions he provided during this time. During my time in Nijmegen and Zürich, my work on decoupling profited from a fruitful collaboration with Aswin Verhoeven, Prof. Helen Geen (University of Nottingham, U.K.), Herbert Zimmermann (MPI für medizinische Forschung, Heidelberg), Dr. Ago Samoson (National Institute of Chemical Physics and Biophysics, Estonia), Dr. Edme Hardy, and Dr. Andreas Detken who were all involved in the decoupling work at different stages and in different ways.

I would also like to thank all the group members of the research groups in Berkeley, Nijmegen, and Zürich who were always open for discussions and questions while working on different projects from mine. The stimulating and very open atmosphere during this whole time was an important factor for my decision to stay in solid-state NMR and academia.

A special thanks goes to Dr. Susan M. De Paul for carefully reading the manuscript and correcting the "German" English into proper English. Of course, all remaining errors are mine. 
